\title{
Triangular integrals for 2-, 3- and 4-variable functions
}

Kiyohisa Tokunaga*

\section{"Correspondence:}

kiyohisa@bene.fit.ac.jp

Laboratory of Information Science,

Fukuoka Institute of Technology,

Wajiro, Higashi-ku, Fukuoka,

811-0295, Japan

\begin{abstract}
The triangular integrals for 2-, 3- and 4-variable functions are respectively and precisely defined as the single limits of double, triple, and quadruple sums in detail. A corollary of the divergence theorem in each dimension is useful to determine the triangular integral value. The indices of the sequence of the integrand must coincide with those of the corresponding integral variable to calculate the correct triangular integral value. In a triangular triple integral, one kind of two sets of increments is inappropriate for the convergence of numerical values, but the other kind is able to calculate numerical values by a computer algebra system.
\end{abstract}

\section{Introduction}

The primary theme of this article is a double integral for a 2-variable function $p=p(x, y)$ in a domain $D$ on the $2 \mathrm{D}$ plane. A double integral is usually regarded as a rectangular double integral. The calculation process of the rectangular double integral $[1,2]$ is conventionally defined as the double limits at infinity of double independent sums, $n \rightarrow \infty$ for $i=1,2, \ldots, n$ and $k \rightarrow \infty$ for $j=1,2, \ldots, k$, of rectangularly divided areas by

$$
\iint_{D} p(x, y) d x d y=\lim _{n \rightarrow \infty} \sum_{i=1}^{n} \lim _{k \rightarrow \infty} \sum_{j=1}^{k} p\left(x_{i}, y_{j}\right) \Delta x_{i} \Delta y_{j},
$$

where $\Delta x_{i}=x_{i}-x_{i-1}$ and $\Delta y_{j}=y_{j}-y_{j-1}$. On the other hand, a triangle mesh or triangular mesh is widely used in the computer graphics. In addition to introducing triangular elements in the finite element method [3], a combination of a triangular area method and double dependent series was applied to sweep all of the area [4]. Proencą and Filipe showed the advantage of a triangular region in comparison with rectangular one for a finite area in real-time face detection. They only investigated a finite sum of finite triangular areas, but our theory of the triangular integral $[5,6]$ treats infinite sum of infinitesimal triangular areas. Moreover, it involves the total differential and the antisymmetric property [7]. The calculation process of the triangular double integral on the 2D plane, where triangular double integral is expressed as (2.36), has not been defined even in the previous article [6]. A corollary of the divergence theorem on the 2D plane is useful to determine the triangular double integral value. The indices of the sequence of the integrand must coincide with those of the corresponding integral variable to calculate the correct triangular integral value. The calculation process of the triangular double integral for a 2-variable function on the $2 \mathrm{D}$ plane is precisely defined as the single limit of double dependent sums

○2014 Tokunaga; licensee Springer. This is an Open Access article distributed under the terms of the Creative Commons Attribution License (http://creativecommons.org/licenses/by/2.0), which permits unrestricted use, distribution, and reproduction in any medium, provided the original work is properly cited. 
by (2.30) in Definition 3. Applying Definition 3, it is able to calculate numerical values by a computer algebra system in Example 1.

The secondary theme of this article is a triple integral for a 3-variable function $q=$ $q(x, y, z)$ in a domain $D$ in the 3D space. A triple integral is usually regarded as a rectangular triple integral. The calculation process of the rectangular triple integral is conventionally defined as the triple limits at infinities of triple independent sums, $n \rightarrow \infty$ for $i=1,2, \ldots, n$ and $k \rightarrow \infty$ for $j=1,2, \ldots, k$ and $m \rightarrow \infty$ for $l=1,2, \ldots, m$, of rectangularly divided volumes by

$$
\iiint_{D} q(x, y, z) d x d y d z=\lim _{n \rightarrow \infty} \sum_{i=1}^{n} \lim _{k \rightarrow \infty} \sum_{j=1}^{k} \lim _{m \rightarrow \infty} \sum_{l=1}^{m} q\left(x_{i}, y_{j}, z_{l}\right) \Delta x_{i} \Delta y_{j} \Delta z_{l}
$$

where $\Delta x_{i}=x_{i}-x_{i-1}, \Delta y_{j}=y_{j}-y_{j-1}$, and $\Delta z_{l}=z_{l}-z_{l-1}$. As shown in the previous article [6], a triangular triple integral can be expressed as (3.65). A corollary of the divergence theorem in the 3D space is useful to determine the triangular triple integral value. In this calculation process of the triangular triple integral, new difficulty has arisen. For the integrand of the divergence theorem in the 3D space, there two alternative ways of decomposition of two kinds of double sequences $\left(X^{\mu}\right)_{j, k}$ and $\left(X^{\mu}\right)_{k, j}$ for $\mu=1,2,3$ and $j=1,2, \ldots, k$ and $k=1,2, \ldots, n$. One way is used in the previous article [6], the other way is used as (3.39) and (3.43) in this article. One kind of the two sets of increments $\left\{\Delta_{i}\left(x^{\gamma}\right)_{i, 0}, \Delta_{h}\left(x^{\gamma}\right)_{j, h}\right\}$ and $\left\{\Delta_{i}\left(x^{\gamma}\right)_{0, i}, \Delta_{h}\left(x^{\gamma}\right)_{h, j}\right\}$ for $\gamma=1,2,3$ and $i=1,2, \ldots, j$ and $j, h=1,2, \ldots, k$ used in the previous article [6] is inappropriate for convergence of integral values since it is unable to calculate numerical values by a computer algebra system in Example 2. However, the other kind of two sets of increments

1. $\left\{\Delta_{h}\left(x^{\gamma}\right)_{0, h}, \Delta_{i}\left(x^{\gamma}\right)_{i, k}\right\}$, derived from (3.39),

2. $\left\{\Delta_{h}\left(x^{\gamma}\right)_{h, 0}, \Delta_{i}\left(x^{\gamma}\right)_{k, i}\right\}$, derived from (3.43) for $\gamma=1,2,3$ and $i=1,2, \ldots, j$ and $h=1,2, \ldots, k$ and $k=1,2, \ldots, n$ used in this article is able to calculate numerical values by a computer algebra system in Example 2. We formulate the divergence theorem in the 3D space and related corollary based on the appropriate two sets of increments in this article. The calculation process of the triangular triple integral for a 3-variable function in the 3D space is precisely defined as the single limit of triple dependent sums by (3.52) in Definition 6 .

The tertiary theme of this article is quadruple integral for a 4-variable function $w=$ $w(t, x, y, z)$ in a domain $D$ in the $4 \mathrm{D}$ time-space. Quadruple integral is usually regarded as the rectangular quadruple integral. The calculation process of the rectangular quadruple integral is conventionally defined as the quadruple limits at infinities of quadruple independent sums, $s \rightarrow \infty$ for $h=1,2, \ldots, s$ and $n \rightarrow \infty$ for $i=1,2, \ldots, n$ and $k \rightarrow \infty$ for $j=1,2, \ldots, k$ and $m \rightarrow \infty$ for $l=1,2, \ldots, m$, of rectangularly divided hyper-volumes by

$$
\begin{aligned}
& \iiint \int_{D} w(t, x, y, z) d t d x d y d z \\
& \quad=\lim _{s \rightarrow \infty} \sum_{h=1}^{s} \lim _{n \rightarrow \infty} \sum_{i=1}^{n} \lim _{k \rightarrow \infty} \sum_{j=1}^{k} \lim _{m \rightarrow \infty} \sum_{l=1}^{m} w\left(t_{h}, x_{i}, y_{j}, z_{l}\right) \Delta t_{h}, \Delta x_{i} \Delta y_{j} \Delta z_{l},
\end{aligned}
$$

where $\Delta t_{h}=t_{h}-t_{h-1}, \Delta x_{i}=x_{i}-x_{i-1}, \Delta y_{j}=y_{j}-y_{j-1}$ and $\Delta z_{l}=z_{l}-z_{l-1}$. The triangular quadruple integral is expressed as (4.110). A corollary of the divergence theorem in 
the 4D time-space is useful to determine the triangular quadruple integral value. Increments in the 4D time-space are replaced for the convergence of the triangular integral value. One kind of six sets of increments $\left\{\Delta_{m}\left(x^{\delta}\right)_{0,0, m}, \Delta_{i}\left(x^{\delta}\right)_{i, 0, l}, \Delta_{h}\left(x^{\delta}\right)_{j, h, l}\right\},\left\{\Delta_{m}\left(x^{\delta}\right)_{0, m, 0}\right.$, $\left.\Delta_{i}\left(x^{\delta}\right)_{i, l, 0}, \Delta_{h}\left(x^{\delta}\right)_{j, l, h}\right\},\left\{\Delta_{m}\left(x^{\delta}\right)_{m, 0,0}, \Delta_{i}\left(x^{\delta}\right)_{l, i, 0}, \Delta_{h}\left(x^{\delta}\right)_{l, j, h}\right\},\left\{\Delta_{m}\left(x^{\delta}\right)_{m, 0,0}, \Delta_{i}\left(x^{\delta}\right)_{l, 0, i}, \Delta_{h}\left(x^{\delta}\right)_{l, h, j}\right\}$, $\left\{\Delta_{m}\left(x^{\delta}\right)_{0, m, 0}, \Delta_{i}\left(x^{\delta}\right)_{0, l, i}, \Delta_{h}\left(x^{\delta}\right)_{h, l, j}\right\}$ and $\left\{\Delta_{m}\left(x^{\delta}\right)_{0,0, m}, \Delta_{i}\left(x^{\delta}\right)_{0, i, l}, \Delta_{h}\left(x^{\delta}\right)_{h, j, l}\right\}$ for $\delta=0,1,2,3$ and $m=1,2, \ldots, l$ and $i, l=1,2, \ldots, j$ and $j, h=1,2, \ldots, k$ used in the previous article [6] is inappropriate since they are the extension of the inappropriate increments $\left\{\Delta_{i}\left(x^{\gamma}\right)_{i, 0}, \Delta_{h}\left(x^{\gamma}\right)_{j, h}\right\}$ and $\left\{\Delta_{i}\left(x^{\gamma}\right)_{0, i}, \Delta_{h}\left(x^{\gamma}\right)_{h, j}\right\}$ for $\gamma=1,2,3$ and $i=1,2, \ldots, j$ and $j, h=$ $1,2, \ldots, k$ to in the $4 \mathrm{D}$ time-space. For the integrand of the divergence theorem in the 4D time-space, there are six alternative ways of decomposition of six kinds of triple sequences $\left(X^{\mu}\right)_{j, k, l},\left(X^{\mu}\right)_{j, l, k},\left(X^{\mu}\right)_{l, j, k},\left(X^{\mu}\right)_{l, k, j},\left(X^{\mu}\right)_{k, l, j}$, and $\left(X^{\mu}\right)_{k, j, l}$ for $\mu=0,1,2,3$ and $l=1,2, \ldots, j$ and $j=1,2, \ldots, k$ and $k=1,2, \ldots, n$. Extending the appropriate set of the increments $\left\{\Delta_{h}\left(x^{\gamma}\right)_{0, h}, \Delta_{i}\left(x^{\gamma}\right)_{i, k}\right\}$ and $\left\{\Delta_{h}\left(x^{\gamma}\right)_{h, 0}, \Delta_{i}\left(x^{\gamma}\right)_{k, i}\right\}$ for $\gamma=1,2,3$ and $i=1,2, \ldots, j$ and $j, h=1,2, \ldots, k$ and $k=1,2, \ldots, n$ in the 3D space to in the 4D time-space, another kind of six sets of increments

1. $\left\{\Delta_{h}\left(x^{\delta}\right)_{0, h, 0}, \Delta_{i}\left(x^{\delta}\right)_{i, k, 0}, \Delta_{m}\left(x^{\delta}\right)_{j, k, m}\right\}$, derived from (4.92),

2. $\left\{\Delta_{h}\left(x^{\delta}\right)_{0,0, h}, \Delta_{i}\left(x^{\delta}\right)_{i, 0, k}, \Delta_{m}\left(x^{\delta}\right)_{j, m, k}\right\}$, derived from (4.97),

3. $\left\{\Delta_{h}\left(x^{\delta}\right)_{0,0, h}, \Delta_{i}\left(x^{\delta}\right)_{0, i, k}, \Delta_{m}\left(x^{\delta}\right)_{m, j, k}\right\}$, derived from (4.98),

4. $\left\{\Delta_{h}\left(x^{\delta}\right)_{0, h, 0}, \Delta_{i}\left(x^{\delta}\right)_{0, k, i}, \Delta_{m}\left(x^{\delta}\right)_{m, k, j}\right\}$, derived from (4.99),

5. $\left\{\Delta_{h}\left(x^{\delta}\right)_{h, 0,0}, \Delta_{i}\left(x^{\delta}\right)_{k, 0, i}, \Delta_{m}\left(x^{\delta}\right)_{k, m, j}\right\}$, derived from (4.100),

6. $\left\{\Delta_{h}\left(x^{\delta}\right)_{h, 0,0}, \Delta_{i}\left(x^{\delta}\right)_{k, i, 0}, \Delta_{m}\left(x^{\delta}\right)_{k, j, m}\right\}$, derived from (4.101)

for $\delta=0,1,2,3$ and $m=1,2, \ldots, l$ and $i=1,2, \ldots, j$ and $h, j=1,2, \ldots, k$ and $k=1,2, \ldots, n$ is derived in this article. This kind of six sets of increments is used in Definition 9. We formulate the divergence theorem in the $4 \mathrm{D}$ time-space and related corollary based on the appropriate 6 sets of increments in this article. The calculation process of the triangular quadruple integral for a 4-variable function in the 4D time-space is precisely defined as the single limit of quadruple dependent sums by (4.111) in Definition 9.

This article is basically about the calculation processes of the triangular double, triple and quadruple integrals for 2-, 3- and 4-variable functions. This article also includes revisions of the divergence theorems and the related corollaries based on the appropriate increments of the double and the triple sequences in the calculation processes of the triangular triple and quadruple integrals for 3- and 4-variable functions.

This article is structured as follows. In Section 2, the divergence theorem of the triangular integral and a related corollary on the $2 \mathrm{D}$ plane are reviewed. The calculation process of the triangular double integral for a 2-variable function is precisely defined in detail. In Section 3, the divergence theorem of the triangular integral and a related corollary in the $3 \mathrm{D}$ space are revised based on the appropriate increments of the double sequence. The calculation process of the triangular triple integral for a 3-variable function is precisely defined in detail. In Section 4, the divergence theorem of the triangular integral and a related corollary in the 4D time-space are revised based on the appropriate increments of the triple sequence. The calculation process of the triangular quadruple integral for a 4-variable function is precisely defined in detail.

\section{Triangular double integral on the 2D plane}

One kind of combined and antisymmetric finite line element vectors on the 2D plane is reviewed in Section 2.1. The triangular double integral for a 2-variable function is shown 
in Section 2.2. Component representation and an example of it for a 2-variable function are shown in Section 2.3. In the following, the Cartesian coordinates are denoted $x^{1}=x$ and $x^{2}=y$.

\subsection{One kind of finite line element vector on the 2D plane}

For triangular double integral, the following increments of single sequence of points on the $2 \mathrm{D}$ plane are introduced.

The increments of single sequence of points $\left(x^{\alpha}\right)_{k}$ are denoted as follows:

$$
\Delta\left(x^{\alpha}\right)_{k} \equiv\left(x^{\alpha}\right)_{k}-\left(x^{\alpha}\right)_{k-1}
$$

for $\alpha=1,2$ and $k=1,2, \ldots, n$.

The finite line element vector $\Delta\left(l^{\alpha}\right)_{k}$ for $\alpha=1,2$ and $k=1,2, \ldots, n$ is introduced as

$$
\Delta\left(l^{\alpha}\right)_{k}=-\Delta\left(x^{\alpha}\right)_{k}
$$

The antisymmetric symbol on the $2 \mathrm{D}$ plane is

$$
\varepsilon^{\alpha \beta}=\varepsilon_{\alpha \beta}= \begin{cases}+1 & \text { for } \varepsilon^{12}=\varepsilon_{12} \\ -1 & \text { for } \varepsilon^{21}=\varepsilon_{21}, \\ 0 & \text { otherwise }\end{cases}
$$

Using the antisymmetric symbol in (2.3), the antisymmetric finite line element vector $\left(\Delta l_{\mu}\right)_{k}$ for $\mu=1,2$ and $k=1,2, \ldots, n$ is introduced as

$$
\left(\Delta l_{\mu}\right)_{k}=\varepsilon_{\alpha \mu} \Delta\left(l^{\alpha}\right)_{k}
$$

and expressed as

$$
\left(\Delta l_{\mu}\right)_{k}=-\varepsilon_{\alpha \mu} \Delta\left(x^{\alpha}\right)_{k}
$$

where the index is summed over $\alpha=1,2$.

For example, we consider the case that the boundary of the domain is an ellipse:

$$
\frac{x^{2}}{a^{2}}+\frac{y^{2}}{b^{2}}=1
$$

where $a>0$ and $b>0$. The following is shown in The curl theorem of a triangular integral [5].

The Cartesian coordinates of the sequence of points $\left(x_{j}, y_{j}\right)$ for $j=0,1,2, \ldots, k$, and $\left(x_{k}, y_{k}\right)$ for $k=0,1,2, \ldots, n$ on the ellipse (2.6) are respectively expressed as

$$
\begin{aligned}
& x_{j}=a \cos \varphi_{j}, \quad y_{j}=b \sin \varphi_{j}, \\
& x_{k}=a \cos \varphi_{k}, \quad y_{k}=b \sin \varphi_{k},
\end{aligned}
$$


where angular arithmetic sequences $\varphi_{j}$ and $\varphi_{k}$ are respectively

$$
\begin{aligned}
\varphi_{j} & =2 \frac{j}{n} \pi, \\
\varphi_{k} & =2 \frac{k}{n} \pi .
\end{aligned}
$$

\subsection{Triangular double integral for a 2-variable function}

Assume that $D$ is a domain and $\partial D$ is the boundary of the domain on the 2D plane, expressed in the Cartesian coordinates $(x, y) \in \mathbb{R}^{2}$. Let $X^{1}=X=X(x, y)$ and $X^{2}=Y=Y(x, y)$ be partially differentiable functions with respect to $x^{1}=x$ and $x^{2}=y$ in $D$. There is only $1 !=1$ kind of single sequence

$$
\left(X^{\mu}\right)_{k}=\left(X_{k}, Y_{k}\right)
$$

for $\mu=1,2$ and $k=0,1,2, \ldots, n$. There are $2 !=2$ sets of possible partial increments for a 2 -variable function.

The total increments of $\left(X^{\mu}\right)_{j}$ for $\mu=1,2$ and $j=1,2, \ldots, k$ are denoted

$$
\begin{aligned}
\Delta\left(X^{\mu}\right)_{j} & \equiv\left(X^{\mu}\right)_{j}-\left(X^{\mu}\right)_{j-1} \\
& =X^{\mu}\left(x_{j}, y_{j}\right)-X^{\mu}\left(x_{j-1}, y_{j-1}\right) .
\end{aligned}
$$

The increments of $\left(x^{\beta}\right)_{j}$ for $\beta=1,2$ and $j=1,2, \ldots, k$ are denoted

$$
\Delta\left(x^{\beta}\right)_{j} \equiv\left(x^{\beta}\right)_{j}-\left(x^{\beta}\right)_{j-1} .
$$

The sets of possible partial increments of $\left(X^{\mu}\right)_{j}$ for $\mu=1,2$ and $j=1,2, \ldots, k$ are denoted

$$
\begin{aligned}
& \Delta X^{\mu}\left[\Delta x_{j}\right]=X^{\mu}\left(x_{j}, y_{j}\right)-X^{\mu}\left(x_{j-1}, y_{j}\right), \\
& \Delta X^{\mu}\left[\Delta y_{j}\right]=X^{\mu}\left(x_{j-1}, y_{j}\right)-X^{\mu}\left(x_{j-1}, y_{j-1}\right) .
\end{aligned}
$$

Lemma 1 Let $X^{\mu}=X^{\mu}\left(x^{\beta}\right)$ be partially differentiable functions with respect to $x^{\beta}$ for $\beta, \mu=$ 1,2. The following holds:

$$
\left(X^{\mu}\right)_{k}=\left(X^{\mu}\right)_{0}+\sum_{j=1}^{k} \frac{\Delta X^{\mu}\left[\Delta\left(x^{\beta}\right)_{j}\right]}{\Delta\left(x^{\beta}\right)_{j}} \Delta\left(x^{\beta}\right)_{j}
$$

for $\mu=1,2$ and $k=1,2, \ldots, n$, where the index is summed over $\beta=1,2$.

Proof The proof of this lemma was shown in the previous article [6].

Our triangular single and double integrals and the divergence theorem on the 2D plane are shown as follows.

Definition 1 The triangular line single integral on 2D plane $\int_{\partial D} X^{\mu} d l_{\mu}$ is defined as

$$
\int_{\partial D} X^{\mu} d l_{\mu}=\lim _{n \rightarrow \infty} \sum_{k=1}^{n}\left(X^{\mu}\right)_{k}\left(\Delta l_{\mu}\right)_{k}
$$


where $\partial D$ is the boundary of a domain, $d l_{\mu}$ is the antisymmetric infinitesimal line element vector and the index is summed over $\mu=1,2$.

Definition 2 The triangular double integral for integrands of which are partial differentials on the 2D plane $\iint_{D} \frac{\partial X^{\prime \mu}}{\partial x^{\prime \beta}} d x^{\prime \beta} d l_{\mu}$ is defined as

$$
\iint_{D} \frac{\partial X^{\prime \mu}}{\partial x^{\prime \beta}} d x^{\prime \beta} d l_{\mu}=\lim _{n \rightarrow \infty} \sum_{k=1}^{n} \sum_{j=1}^{k} \frac{\Delta X^{\mu}\left[\Delta\left(x^{\beta}\right)_{j}\right]}{\Delta\left(x^{\beta}\right)_{j}} \Delta\left(x^{\beta}\right)_{j}\left(\Delta l_{\mu}\right)_{k}
$$

where $D$ is a domain and the indices are summed over $\beta, \mu=1,2$.

The following proposition is necessary for the condition (2.25) in Theorem 1.

Proposition 1 Denote constants as $C^{1}=C^{x}$ and $C^{2}=C^{y}$, then

$$
C^{\mu} \int_{\partial D} d l_{\mu}=C^{\mu} \lim _{n \rightarrow \infty} \sum_{k=1}^{n}\left(\Delta l_{\mu}\right)_{k}
$$

holds, where the index is summed over $\mu=1,2$.

Proof In the case of $X^{1}=1$ and $X^{2}=0$, Definition 1 is reduced to

$$
\int_{\partial D} d l_{x}=\lim _{n \rightarrow \infty} \sum_{k=1}^{n}\left(\Delta l_{x}\right)_{k}
$$

In the case of $X^{1}=0$ and $X^{2}=1$, Definition 1 is reduced to

$$
\int_{\partial D} d l_{y}=\lim _{n \rightarrow \infty} \sum_{k=1}^{n}\left(\Delta l_{y}\right)_{k}
$$

A linear combination of (2.20) and (2.21) is

$$
C^{x} \int_{\partial D} d l_{x}+C^{y} \int_{\partial D} d l_{y}=C^{x} \lim _{n \rightarrow \infty} \sum_{k=1}^{n}\left(\Delta l_{x}\right)_{k}+C^{y} \lim _{n \rightarrow \infty} \sum_{k=1}^{n}\left(\Delta l_{y}\right)_{k} .
$$

We show that (2.24) holds for a closed curve in the following.

The sum of $\left(\Delta l_{\mu}\right)_{k}$ in (2.5) over $k=1,2, \ldots, n$ for $\mu=1,2$ is

$$
\begin{aligned}
\sum_{k=1}^{n}\left(\Delta l_{\mu}\right)_{k} & =-\varepsilon_{\alpha \mu} \sum_{k=1}^{n} \Delta\left(x^{\alpha}\right)_{k} \\
& =-\varepsilon_{\alpha \mu}\left[\left(x^{\alpha}\right)_{n}-\left(x^{\alpha}\right)_{0}\right],
\end{aligned}
$$

where the index is summed over $\alpha=1,2$. In the case of a closed curve, i.e., $x_{0}=x_{n}$ and $y_{0}=y_{n}$, it satisfies

$$
\sum_{k=1}^{n}\left(\Delta l_{\mu}\right)_{k}=0
$$


The following is the refined version of the theorem shown in The divergence theorem of a triangular integral [6].

Theorem 1 (The divergence theorem of the triangular integral on the 2D plane) Assume that $\partial D$ is a piecewise smooth curve of the equation and $D$ is the region inside and on $\partial D$ on the $2 D$ plane, expressed in the Cartesian coordinates $(x, y) \in \mathbb{R}^{2}$. Let $X^{\mu}$ for $\mu=1,2$ be a set of partially differentiable functions with respect to $x^{\nu}$ for $v=1,2$ in $D$, where $X^{\mu}=X^{\mu}\left(x^{\nu}\right)$. In the case of a closed integral path which satisfies

$$
C^{\mu} \int_{\partial D} d l_{\mu}=0
$$

the divergence theorem of the triangular integral on the $2 D$ plane holds:

$$
\oint_{\partial D} X^{\mu} d l_{\mu}=\iint_{D} \frac{\partial X^{\prime \mu}}{\partial x^{\prime \beta}} d x^{\prime \beta} d l_{\mu}
$$

where the indices are summed over $\beta, \mu=1,2$.

Proof Combining (2.16) with (2.4) for the sum of $k=1,2, \ldots, n$, we obtain

$$
\sum_{k=1}^{n}\left(X^{\mu}\right)_{k}\left(\Delta l_{\mu}\right)_{k}=\sum_{k=1}^{n} \sum_{j=1}^{k} \frac{\Delta X^{\mu}\left[\Delta\left(x^{\beta}\right)_{j}\right]}{\Delta\left(x^{\beta}\right)_{j}} \Delta\left(x^{\beta}\right)_{j}\left(\Delta l_{\mu}\right)_{k}+\left(X^{\mu}\right)_{0} \sum_{k=1}^{n}\left(\Delta l_{\mu}\right)_{k},
$$

where the indices are summed over $\beta, \mu=1,2$. Using Proposition 1, (2.25) is rewritten as

$$
\left(X^{\mu}\right)_{0} \lim _{n \rightarrow \infty} \sum_{k=1}^{n}\left(\Delta l_{\mu}\right)_{k}=0
$$

where the index is summed over $\mu=1,2$. The limit at infinity $n \rightarrow \infty$ of (2.27) is expressed as (2.26) by Definitions 1 and 2 under the condition of a closed curve (2.28).

The triangular double integral for a 2-variable function $p=p(x, y)$ on the 2D plane by the infinitesimal area element $d^{2} \sigma$ of the triangular double integral on the 2D plane is given as

$$
\iint_{D} p d^{2} \sigma=\frac{1}{2} \iint_{D} p d x^{\prime \beta} d l_{\beta},
$$

where $D$ is a domain and the index is summed over $\beta=1,2$.

The calculation process of the triangular double integral on the 2D plane is precisely defined as follows.

Let $p=p(x, y)$ be a piecewise smooth function on the 2D plane, expressed in the Cartesian coordinates $(x, y) \in \mathbb{R}^{2}$.

Definition 3 The triangular double integral for a 2-variable function $p=p(x, y)$ on the 2D plane $\frac{1}{2} \iint_{D} p d x^{\prime \beta} d l_{\beta}$ is defined as

$$
\frac{1}{2} \iint_{D} p d x^{\prime \beta} d l_{\beta}=\frac{1}{2} \lim _{n \rightarrow \infty} \sum_{k=1}^{n} \sum_{j=1}^{k} p\left(\left(x^{\alpha}\right)_{k},\left(x^{\beta}\right)_{j}\right) \Delta\left(x^{\beta}\right)_{j} \Delta\left(l_{\beta}\right)_{k}
$$


for $\alpha \neq \beta$ and $\alpha=1,2$ as the indices of variables of function $p=p(x, y)$, where $D$ is a domain and the index is summed over $\beta=1,2$.

The following is a corollary of the divergence theorem on the 2D plane.

Corollary 1 (A corollary of Theorem 1) Assume that $\partial D$ is a piecewise smooth curve of the equation and $D$ is the region inside and on $\partial D$ on the $2 D$ plane, expressed in the Cartesian coordinates $(x, y) \in \mathbb{R}^{2}$. Let $X^{\mu}$ for $\mu=1,2$ be a set of partially differentiable functions with respect to $x^{\nu}$ for $v=1,2$ in $D$, where $X^{\mu}=X^{\mu}\left(x^{\nu}\right)$. In the case of

$$
\oint_{\partial D} X^{\mu} d l_{\mu}=\iint_{D} p d^{2} \sigma
$$

where $p=p(x, y)$ is a 2-variable function and the index is summed over $\mu=1,2$, the following holds:

$$
\frac{\partial X^{\mu}}{\partial x^{\mu}}=p
$$

Proof Substituting (2.26) and (2.29) into (2.31), we obtain

$$
\iint_{D} \frac{\partial X^{\prime \mu}}{\partial x^{\prime \beta}} d x^{\prime \beta} d l_{\mu}=\frac{1}{2} \iint_{D} p d x^{\prime \beta} d l_{\beta},
$$

where the indices are summed over $\beta, \mu=1,2$. Substituting (2.18) and (2.30) into (2.33), it is expressed as

$$
\begin{aligned}
& \lim _{n \rightarrow \infty} \sum_{k=1}^{n} \sum_{j=1}^{k} \frac{\Delta X^{\mu}\left[\Delta\left(x^{\beta}\right)_{j}\right]}{\Delta\left(x^{\beta}\right)_{j}} \Delta\left(x^{\beta}\right)_{j} \varepsilon_{\alpha \mu}\left(\Delta l^{\alpha}\right)_{k} \\
& =\frac{1}{2} \lim _{n \rightarrow \infty} \sum_{k=1}^{n} \sum_{j=1}^{k} p\left(\left(x^{\alpha}\right)_{k},\left(x^{\beta}\right)_{j}\right) \Delta\left(x^{\beta}\right)_{j} \varepsilon_{\alpha \beta} \Delta\left(l^{\alpha}\right)_{k^{\prime}}
\end{aligned}
$$

where the indices are summed over $\alpha, \beta, \mu=1,2$. In order for (2.34) to hold for any value of integral variables, the following $1 \times 1$ ! = 1 kind of formula for $\alpha, \beta=1,2$ and $j=1,2, \ldots, k$ and $k=1,2, \ldots, n$ is required:

$$
\lim _{\Delta\left(x^{\beta}\right)_{j} \rightarrow 0} \frac{\Delta X^{\mu}\left[\Delta\left(x^{\beta}\right)_{j}\right]}{\Delta\left(x^{\beta}\right)_{j}} \varepsilon_{\alpha \mu}=p\left(\left(x^{\alpha}\right)_{k},\left(x^{\beta}\right)_{j}\right) \frac{1}{2} \varepsilon_{\alpha \beta},
$$

where the index is summed over $\mu=1,2$.

On the 2D plane, $\varepsilon_{\alpha \mu} \varepsilon^{\alpha \beta}=\delta_{\mu}^{\beta}$ holds for $\beta, \mu=1$, 2, where the index is summed over $\alpha=1,2$. We therefore obtain $\frac{1}{2} \varepsilon_{\alpha \beta} \varepsilon^{\alpha \beta}=1$, where the indices are summed over $\alpha, \beta=1,2$. Multiplying by $\varepsilon^{\alpha \beta}$ both sides of (2.35), it is reduced to the differential equation (2.32).

\subsection{Component representation and an example of it for a 2-variable function}

In component representation, (2.29) is expressed as

$$
\begin{aligned}
\iint_{D} p d^{2} \sigma & =\frac{1}{2} \iint_{D} p d x^{\prime} d l_{x}+\frac{1}{2} \iint_{D} p d y^{\prime} d l_{y} \\
& =\frac{1}{2} \iint_{D} p d x^{\prime} d y-\frac{1}{2} \iint_{D} p d x d y^{\prime}
\end{aligned}
$$


Using (2.30) in Definition 3, each component of (2.36) is

$$
\begin{aligned}
& \frac{1}{2} \iint_{D} p d x^{\prime} d l_{x}=+\frac{1}{2} \iint_{D} p\left(x^{\prime}, y\right) d x^{\prime} d y=+\frac{1}{2} \lim _{n \rightarrow \infty} \sum_{k=1}^{n} \sum_{j=1}^{k} p\left(x_{j}, y_{k}\right) \Delta x_{j} \Delta y_{k} \\
& \frac{1}{2} \iint_{D} p d y^{\prime} d l_{y}=-\frac{1}{2} \iint_{D} p\left(x, y^{\prime}\right) d x d y^{\prime}=-\frac{1}{2} \lim _{n \rightarrow \infty} \sum_{k=1}^{n} \sum_{j=1}^{k} p\left(x_{k}, y_{j}\right) \Delta x_{k} \Delta y_{j} .
\end{aligned}
$$

In Corollary $1,(2.31)$ is expressed as

$$
\oint_{\partial D}(X d y-Y d x)=\iint_{D} p d^{2} \sigma
$$

and (2.32) is expressed as

$$
\frac{\partial X}{\partial x}+\frac{\partial Y}{\partial y}=p .
$$

We show an example of Corollary 1 in the following.

Example 1 In the case of

$$
X(x, y)=x y^{2}, \quad Y(x, y)=x^{2} y
$$

Substituting (2.41) into (2.40), we obtain

$$
p(x, y)=x^{2}+y^{2}
$$

The boundary of the domain is an ellipse (2.6).

1. The value of the left-hand side of (2.39) is

$$
\begin{aligned}
\oint_{\partial D}(X d y-Y d x) & =\oint_{\frac{x^{2}}{a^{2}}+\frac{y^{2}}{b^{2}}=1}\left(x y^{2} d y-x^{2} y d x\right) \\
& =\lim _{n \rightarrow \infty} \sum_{k=1}^{n} x_{k} y_{k}^{2} \Delta y_{k}-\lim _{n \rightarrow \infty} \sum_{k=1}^{n} x_{k}^{2} y_{k} \Delta x_{k} \\
& =\frac{1}{4} \pi a b\left(a^{2}+b^{2}\right) .
\end{aligned}
$$

See (A.1) and (A.2) in Appendix 1 for calculations in detail.

2 . The value of the right-hand side of (2.39) is

$$
\begin{aligned}
\iint_{D} p d^{2} \sigma & =\frac{1}{2} \iint_{\frac{x^{2}}{a^{2}}+\frac{y^{2}}{b^{2}} \leq 1}\left[\left(x^{\prime}\right)^{2}+y^{2}\right] d x^{\prime} d y-\frac{1}{2} \iint_{\frac{x^{2}}{a^{2}}+\frac{y^{2}}{b^{2}} \leq 1}\left[x^{2}+\left(y^{\prime}\right)^{2}\right] d x d y^{\prime} \\
& =\frac{1}{2} \lim _{n \rightarrow \infty} \sum_{k=1}^{n} \sum_{j=1}^{k}\left(x_{j}^{2}+y_{k}^{2}\right) \Delta x_{j} \Delta y_{k}-\frac{1}{2} \lim _{n \rightarrow \infty} \sum_{k=1}^{n} \sum_{j=1}^{k}\left(x_{k}^{2}+y_{j}^{2}\right) \Delta x_{k} \Delta y_{j} \\
& =\frac{1}{4} \pi a b\left(a^{2}+b^{2}\right) .
\end{aligned}
$$

See (A.3), (A.4), (A.5), and (A.6) in Appendix 1 for calculations in detail. 
We thus see the coincidence of the value of (2.43) and that of (2.44).

Equation (2.39) is verified also in the case of $X(x, y)=x^{3} y^{4}$ and $Y(x, y)=x^{4} y^{3}$.

We consider the following approximation formula of (2.39) for $1 \ll n<\infty$,

$$
\begin{aligned}
& \sum_{k=1}^{n}\left[X\left(x_{k}, y_{k}\right) \Delta y_{k}-Y\left(x_{k}, y_{k}\right) \Delta x_{k}\right] \\
& \simeq \frac{1}{2} \sum_{k=1}^{n} \sum_{j=1}^{k}\left[p\left(x_{j}, y_{k}\right) \Delta x_{j} \Delta y_{k}-p\left(x_{k}, y_{j}\right) \Delta x_{k} \Delta y_{j}\right] .
\end{aligned}
$$

In the case of $n \rightarrow \infty,(2.45)$ coincides with (2.39). It is verified by (2.43) and (2.44) in Example 1. An approximation formula, see (2.39), for Example 1 is

$$
\sum_{k=1}^{n}\left(x_{k} y_{k}^{2} \Delta y_{k}-x_{k}^{2} y_{k} \Delta x_{k}\right) \simeq \frac{1}{2} \sum_{k=1}^{n} \sum_{j=1}^{k}\left[\left(x_{j}^{2}+y_{k}^{2}\right) \Delta x_{j} \Delta y_{k}-\left(x_{k}^{2}+y_{j}^{2}\right) \Delta x_{k} \Delta y_{j}\right] .
$$

The left- and the right-hand sides of (2.46), respectively expressed as $L$ and $R$, are shown in Table 1 and plotted in Figure 1, where $a=b=1$.

Table 1 Approximate values of Example 1

\begin{tabular}{llll}
\hline $\boldsymbol{n}$ & $\boldsymbol{L}$ & $\boldsymbol{R}$ & $\boldsymbol{R}-\boldsymbol{L}$ \\
\hline 2 & 0 & 0 & 0 \\
4 & 1 & 2 & 1 \\
8 & $1.41421356 \ldots$ & $1.82842712 \ldots$ & $0.41421356 \ldots$ \\
16 & $1.53073372 \ldots$ & $1.64725389 \ldots$ & $0.11652016 \ldots$ \\
32 & $1.56072257 \ldots$ & $1.59071142 \ldots$ & $0.02998884 \ldots$ \\
64 & $1.56827424 \ldots$ & $1.57582591 \ldots$ & $0.00755166 \ldots$ \\
128 & $1.57016557 \ldots$ & $1.57205691 \ldots$ & $0.00189133 \ldots$ \\
256 & $1.57063862 \ldots$ & $1.57111167 \ldots$ & $0.00047304 \ldots$ \\
512 & $1.57075690 \ldots$ & $1.57087517 \ldots$ & $0.00011827 \ldots$ \\
1,024 & $1.57078647 \ldots$ & $1.57081603 \ldots$ & $0.00002956 \ldots$ \\
2,048 & $1.57079386 \ldots$ & $1.57080125 \ldots$ & $0.00000739 \ldots$ \\
4,096 & $1.57079571 \ldots$ & $1.57079755 \ldots$ & $0.00000184 \ldots$ \\
8,192 & $1.57079617 \ldots$ & $1.57079663 \ldots$ & $0.00000046 \ldots$ \\
$\vdots$ & $\vdots$ & $\vdots$ & $\vdots$ \\
$\infty$ & $1.57079632 \ldots$ & $1.57079632 \ldots$ & 0 \\
\hline
\end{tabular}

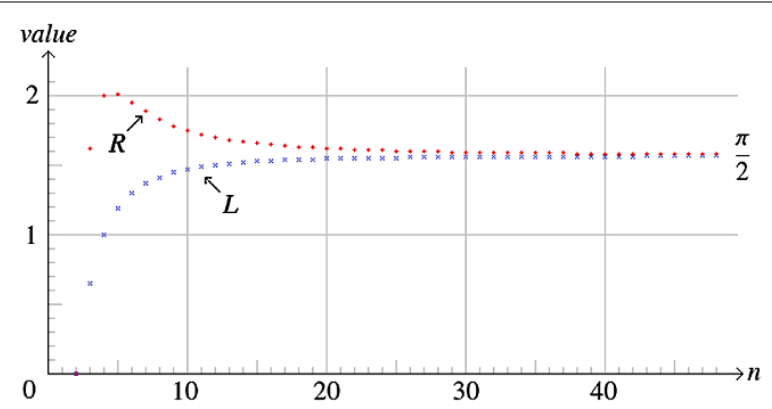

Figure 1 Approximate values of Example 1. 


\section{Triangular triple integral in the 3D space}

Two kinds of combined and antisymmetric finite area element vectors in the 3D space are reviewed in Section 3.1. The triple integral for a 3-variable function is shown in Section 3.2. Component representation and an example of it for a 3-variable function are shown in Section 3.3. In the following, the Cartesian coordinates are denoted $x^{1}=x, x^{2}=y$, and $x^{3}=z$.

\subsection{Two kinds of finite area element vectors in the 3D space}

For a triangular triple integral, the following increments of the double sequence of points in the 3D space are introduced.

1. The increments of the double sequence of points at $(j, k)$ are denoted as follows:

$$
\begin{aligned}
& \Delta_{j}\left(x^{\alpha}\right)_{j, k} \equiv\left(x^{\alpha}\right)_{j, k}-\left(x^{\alpha}\right)_{j-1, k}, \\
& \Delta_{k}\left(x^{\beta}\right)_{j-1, k} \equiv\left(x^{\beta}\right)_{j-1, k}-\left(x^{\beta}\right)_{j-1, k-1}, \\
& \Delta_{k}\left(x^{\alpha}\right)_{j, k} \equiv\left(x^{\alpha}\right)_{j, k}-\left(x^{\alpha}\right)_{j, k-1}, \\
& \Delta_{j}\left(x^{\beta}\right)_{j, k-1} \equiv\left(x^{\beta}\right)_{j, k-1}-\left(x^{\beta}\right)_{j-1, k-1}
\end{aligned}
$$

for $\alpha, \beta=1,2,3$ and $j=1,2, \ldots, k$ and $k=1,2, \ldots, n$.

The first combined finite area element vector $\left(\Delta^{2} \sigma^{\alpha \beta}\right)_{j, k}$ for $\alpha, \beta=1,2,3$ and $j=1,2, \ldots, k$ and $k=1,2, \ldots, n$ is introduced as

$$
\left(\Delta^{2} \sigma^{\alpha \beta}\right)_{j, k}=\Delta_{j}\left(x^{\alpha}\right)_{j, k} \Delta_{k}\left(x^{\beta}\right)_{j-1, k}-\Delta_{k}\left(x^{\alpha}\right)_{j, k} \Delta_{j}\left(x^{\beta}\right)_{j, k-1} .
$$

2. The increments of the double sequence of points at $(k, j)$ are denoted as follows:

$$
\begin{aligned}
& \Delta_{k}\left(x^{\alpha}\right)_{k, j} \equiv\left(x^{\alpha}\right)_{k, j}-\left(x^{\alpha}\right)_{k-1, j}, \\
& \Delta_{j}\left(x^{\beta}\right)_{k-1, j} \equiv\left(x^{\beta}\right)_{k-1, j}-\left(x^{\beta}\right)_{k-1, j-1}, \\
& \Delta_{j}\left(x^{\alpha}\right)_{k, j} \equiv\left(x^{\alpha}\right)_{k, j}-\left(x^{\alpha}\right)_{k, j-1}, \\
& \Delta_{k}\left(x^{\beta}\right)_{k, j-1} \equiv\left(x^{\beta}\right)_{k, j-1}-\left(x^{\beta}\right)_{k-1, j-1}
\end{aligned}
$$

for $\alpha, \beta=1,2,3$ and $j=1,2, \ldots, k$ and $k=1,2, \ldots, n$.

The second combined finite area element vector $\left(\Delta^{2} \sigma^{\alpha \beta}\right)_{k, j}$ for $\alpha, \beta=1,2,3$ and $j=$ $1,2, \ldots, k$ and $k=1,2, \ldots, n$ is introduced as

$$
\left(\Delta^{2} \sigma^{\alpha \beta}\right)_{k, j}=\Delta_{k}\left(x^{\alpha}\right)_{k, j} \Delta_{j}\left(x^{\beta}\right)_{k-1, j}-\Delta_{j}\left(x^{\alpha}\right)_{k, j} \Delta_{k}\left(x^{\beta}\right)_{k, j-1} .
$$

The antisymmetric symbol in the 3D space is

$$
\varepsilon^{\alpha \beta \gamma}=\varepsilon_{\alpha \beta \gamma}= \begin{cases}+1 & \text { for even permutation of }\{1,2,3\} \\ -1 & \text { for odd permutation of }\{1,2,3\} \\ 0 & \text { otherwise. }\end{cases}
$$

Using the antisymmetric symbol in (3.11), the first antisymmetric finite area element vector $\left(\Delta^{2} \sigma_{\mu}\right)_{j, k}$ and the second antisymmetric finite area element vector $\left(\Delta^{2} \sigma_{\mu}\right)_{k, j}$ for 
$\mu=1,2,3$ and $j=1,2, \ldots, k$ and $k=1,2, \ldots, n$ are respectively introduced as

$$
\begin{aligned}
& \left(\Delta^{2} \sigma_{\mu}\right)_{j, k}=\frac{1}{2} \varepsilon_{\alpha \beta \mu}\left(\Delta^{2} \sigma^{\alpha \beta}\right)_{j, k}, \\
& \left(\Delta^{2} \sigma_{\mu}\right)_{k, j}=\frac{1}{2} \varepsilon_{\alpha \beta \mu}\left(\Delta^{2} \sigma^{\alpha \beta}\right)_{k, j},
\end{aligned}
$$

where the indices are summed over $\alpha, \beta=1,2,3$.

1. The first antisymmetric finite area element vector $\left(\Delta^{2} \sigma_{\mu}\right)_{j, k}$ for $\mu=1,2,3$ and $j=$ $1,2, \ldots, k$ and $k=1,2, \ldots, n$ is expressed as

$$
\left(\Delta^{2} \sigma_{\mu}\right)_{j, k}=\frac{1}{2} \varepsilon_{\alpha \beta \mu}\left[\Delta_{j}\left(x^{\alpha}\right)_{j, k} \Delta_{k}\left(x^{\beta}\right)_{j-1, k}-\Delta_{k}\left(x^{\alpha}\right)_{j, k} \Delta_{j}\left(x^{\beta}\right)_{j, k-1}\right]
$$

where the indices are summed over $\alpha, \beta=1,2$, 3. In detail, (3.14) for $\mu=1,2,3$ are respectively written as

$$
\begin{aligned}
\left(\Delta^{2} \sigma_{x}\right)_{j, k}= & \frac{1}{2}\left(\Delta_{j} y_{j, k} \Delta_{k} z_{j-1, k}-\Delta_{k} y_{j, k} \Delta_{j} z_{j, k-1}\right) \\
& -\frac{1}{2}\left(\Delta_{j} z_{j, k} \Delta_{k} y_{j-1, k}-\Delta_{k} z_{j, k} \Delta_{j} y_{j, k-1}\right), \\
\left(\Delta^{2} \sigma_{y}\right)_{j, k}= & \frac{1}{2}\left(\Delta_{j} z_{j, k} \Delta_{k} x_{j-1, k}-\Delta_{k} z_{j, k} \Delta_{j} x_{j, k-1}\right) \\
& -\frac{1}{2}\left(\Delta_{j} x_{j, k} \Delta_{k} z_{j-1, k}-\Delta_{k} x_{j, k} \Delta_{j} z_{j, k-1}\right), \\
\left(\Delta^{2} \sigma_{z}\right)_{j, k}= & \frac{1}{2}\left(\Delta_{j} x_{j, k} \Delta_{k} y_{j-1, k}-\Delta_{k} x_{j, k} \Delta_{j} y_{j, k-1}\right) \\
& -\frac{1}{2}\left(\Delta_{j} y_{j, k} \Delta_{k} x_{j-1, k}-\Delta_{k} y_{j, k} \Delta_{j} x_{j, k-1}\right) .
\end{aligned}
$$

2. The second antisymmetric finite area element vector $\left(\Delta^{2} \sigma_{\mu}\right)_{k, j}$ for $\mu=1,2,3$ and $j=$ $1,2, \ldots, k$ and $k=1,2, \ldots, n$ is expressed as

$$
\left(\Delta^{2} \sigma_{\mu}\right)_{k, j}=\frac{1}{2} \varepsilon_{\alpha \beta \mu}\left[\Delta_{k}\left(x^{\alpha}\right)_{k, j} \Delta_{j}\left(x^{\beta}\right)_{k-1, j}-\Delta_{j}\left(x^{\alpha}\right)_{k, j} \Delta_{k}\left(x^{\beta}\right)_{k, j-1}\right]
$$

where the indices are summed over $\alpha, \beta=1,2,3$. In detail, the equations in (3.18) for $\mu=$ $1,2,3$ are respectively written as

$$
\begin{aligned}
\left(\Delta^{2} \sigma_{x}\right)_{k, j}= & \frac{1}{2}\left(\Delta_{k} y_{k, j} \Delta_{j} z_{k-1, j}-\Delta_{j} y_{k, j} \Delta_{k} z_{k, j-1}\right) \\
& -\frac{1}{2}\left(\Delta_{k} z_{k, j} \Delta_{j} y_{k-1, j}-\Delta_{j} z_{k, j} \Delta_{k} y_{k, j-1}\right), \\
\left(\Delta^{2} \sigma_{y}\right)_{k, j}= & \frac{1}{2}\left(\Delta_{k} z_{k, j} \Delta_{j} x_{k-1, j}-\Delta_{j} z_{k, j} \Delta_{k} x_{k, j-1}\right) \\
& -\frac{1}{2}\left(\Delta_{k} x_{k, j} \Delta_{j} z_{k-1, j}-\Delta_{j} x_{k, j} \Delta_{k} z_{k, j-1}\right), \\
\left(\Delta^{2} \sigma_{z}\right)_{k, j}= & \frac{1}{2}\left(\Delta_{k} x_{k, j} \Delta_{j} y_{k-1, j}-\Delta_{j} x_{k, j} \Delta_{k} y_{k, j-1}\right) \\
& -\frac{1}{2}\left(\Delta_{k} y_{k, j} \Delta_{j} x_{k-1, j}-\Delta_{j} y_{k, j} \Delta_{k} x_{k, j-1}\right) .
\end{aligned}
$$


For example, we consider the case that the boundary of the domain is a sphere:

$$
x^{2}+y^{2}+z^{2}=a^{2},
$$

where $a>0$. The following is shown in The divergence theorem of a triangular integral [6].

1. The Cartesian coordinates $\left(x_{j, k}, y_{j, k}, z_{j, k}\right)$ of the antisymmetric first finite area element vector $\left(\Delta^{2} \sigma_{\mu}\right)_{j, k}$ for $\mu=1,2,3$ and $j=0,1,2, \ldots, k$ and $k=0,1,2, \ldots, n$ on the surface of the sphere (3.22) are respectively expressed as

$$
x_{j, k}=a \sin \theta_{j} \cos \varphi_{k}, \quad y_{j, k}=a \sin \theta_{j} \sin \varphi_{k}, \quad z_{j, k}=a \cos \theta_{j},
$$

where angular arithmetic sequences $\theta_{j}$ and $\varphi_{k}$ are respectively

$$
\theta_{j}=\frac{j}{n} \pi, \quad \varphi_{k}=2 \frac{k}{n} \pi
$$

2. The Cartesian coordinates $\left(x_{k, j}, y_{k, j}, z_{k, j}\right)$ of the antisymmetric second finite area element vector $\left(\Delta^{2} \sigma_{\mu}\right)_{k, j}$ for $\mu=1,2,3$ and $j=0,1,2, \ldots, k$ and $k=0,1,2, \ldots, n$ on the surface of the sphere (3.22) are respectively expressed as

$$
x_{k, j}=a \sin \theta_{k} \cos \varphi_{j}, \quad y_{k, j}=a \sin \theta_{k} \sin \varphi_{j}, \quad z_{k, j}=a \cos \theta_{k}
$$

where angular arithmetic sequences $\theta_{k}$ and $\varphi_{j}$ are respectively

$$
\theta_{k}=\frac{k}{n} \pi, \quad \varphi_{j}=2 \frac{j}{n} \pi
$$

\subsection{Triangular triple integral for a 3-variable function}

Assume that $D$ is a domain and $\partial D$ is the boundary of the domain in the 3D space, expressed in the Cartesian coordinates $(x, y, z) \in \mathbb{R}^{3}$. Let $X^{1}=X=X(x, y, z), X^{2}=Y=$ $Y(x, y, z)$, and $X^{3}=Z=Z(x, y, z)$ be partially differentiable functions with respect to $x^{1}=x$, $x^{2}=y$, and $x^{3}=z$ in $D$. There are $2 !=2$ kinds of the double sequences

$$
\begin{aligned}
& \left(X^{\mu}\right)_{j, k}=\left(X_{j, k}, Y_{j, k}, Z_{j, k}\right), \\
& \left(X^{\mu}\right)_{k, j}=\left(X_{k, j}, Y_{k, j}, Z_{k, j}\right)
\end{aligned}
$$

for $\mu=1,2,3$ and $j=0,1,2, \ldots, k$ and $k=0,1,2, \ldots, n$. There are two alternatives for decomposition of two kinds of double sequences $\left(X^{\mu}\right)_{j, k}$ and $\left(X^{\mu}\right)_{k, j}$ for $\mu=1,2,3$ and $j=1,2, \ldots, k$.

As mentioned in the Introduction, the inappropriate two sets of increments, used in the previous article [6], are replaced by the appropriate kind of two sets of increments $\left\{\Delta_{h}\left(x^{\gamma}\right)_{0, h}, \Delta_{i}\left(x^{\gamma}\right)_{i, k}\right\}$ and $\left\{\Delta_{h}\left(x^{\gamma}\right)_{h, 0}, \Delta_{i}\left(x^{\gamma}\right)_{k, i}\right\}$ for $\gamma=1,2,3$ and $i=1,2, \ldots, j$ and $h=1,2, \ldots, k$ and $k=1,2, \ldots, n$ to calculate the numerical values in Example 2.

The following formulae have been revised based on the appropriate set of increments. In order to prove Theorem $2,\left(X^{\mu}\right)_{j, k}$ and $\left(X^{\mu}\right)_{k, j}$ for $\mu=1,2,3$ and $j=1,2, \ldots, k$ and $k=$ $1,2, \ldots, n$ are respectively modified in Lemmata 2 and 3.

There are $3 !=6$ sets of possible partial increments for a 3-variable function. 
1. The total increments of $\left(X^{\mu}\right)_{0, h}$ for $\mu=1,2,3$ and $h=1,2, \ldots, k$ are denoted

$$
\begin{aligned}
\Delta_{h}\left(X^{\mu}\right)_{0, h} & \equiv\left(X^{\mu}\right)_{0, h}-\left(X^{\mu}\right)_{0, h-1} \\
& =X^{\mu}\left(x_{0, h}, y_{0, h}, z_{0, h}\right)-X^{\mu}\left(x_{0, h-1}, y_{0, h-1}, z_{0, h-1}\right) .
\end{aligned}
$$

The increments of $\left(x^{\gamma}\right)_{0, h}$ for $\gamma=1,2,3$ and $h=1,2, \ldots, k$ are denoted

$$
\Delta_{h}\left(x^{\gamma}\right)_{0, h} \equiv\left(x^{\gamma}\right)_{0, h}-\left(x^{\gamma}\right)_{0, h-1} .
$$

The sets of possible partial increments of $\left(X^{\mu}\right)_{0, h}$ for $\mu=1,2,3$ and $h=1,2, \ldots, k$ are denoted

$$
\begin{aligned}
& \Delta X^{\mu}\left[\Delta_{h} x_{0, h}\right]=X^{\mu}\left(x_{0, h}, y_{0, h}, z_{0, h}\right)-X^{\mu}\left(x_{0, h-1}, y_{0, h}, z_{0, h}\right), \\
& \Delta X^{\mu}\left[\Delta_{h} y_{0, h}\right]=X^{\mu}\left(x_{0, h-1}, y_{0, h}, z_{0, h}\right)-X^{\mu}\left(x_{0, h-1}, y_{0, h-1}, z_{0, h}\right), \\
& \Delta X^{\mu}\left[\Delta_{h} z_{0, h}\right]=X^{\mu}\left(x_{0, h-1}, y_{0, h-1}, z_{0, h}\right)-X^{\mu}\left(x_{0, h-1}, y_{0, h-1}, z_{0, h-1}\right) .
\end{aligned}
$$

2. The total increments of $\left(X^{\mu}\right)_{i, k}$ for $\mu=1,2,3$ and $i=1,2, \ldots, j$ and $k=1,2, \ldots, n$ are denoted

$$
\begin{aligned}
\Delta_{i}\left(X^{\mu}\right)_{i, k} & \equiv\left(X^{\mu}\right)_{i, k}-\left(X^{\mu}\right)_{i-1, k} \\
& =X^{\mu}\left(x_{i, k}, y_{i, k}, z_{i, k}\right)-X^{\mu}\left(x_{i-1, k}, y_{i-1, k}, z_{i-1, k}\right) .
\end{aligned}
$$

The increments of $\left(x^{\gamma}\right)_{i, k}$ for $\gamma=1,2,3$ and $i=1,2, \ldots, j$ and $k=1,2, \ldots, n$ are denoted

$$
\Delta_{i}\left(x^{\gamma}\right)_{i, k} \equiv\left(x^{\gamma}\right)_{i, k}-\left(x^{\gamma}\right)_{i-1, k} .
$$

The sets of possible partial increments of $\left(X^{\mu}\right)_{i, k}$ for $\mu=1,2,3$ and $i=1,2, \ldots, j$ and $k=$ $1,2, \ldots, n$ are denoted

$$
\begin{aligned}
& \Delta X^{\mu}\left[\Delta_{i} x_{i, k}\right]=X^{\mu}\left(x_{i, k}, y_{i, k}, z_{i, k}\right)-X^{\mu}\left(x_{i-1, k}, y_{i, k}, z_{i, k}\right), \\
& \Delta X^{\mu}\left[\Delta_{i} y_{i, k}\right]=X^{\mu}\left(x_{i-1, k}, y_{i, k}, z_{i, k}\right)-X^{\mu}\left(x_{i-1, k}, y_{i-1, k}, z_{i, k}\right), \\
& \Delta X^{\mu}\left[\Delta_{i} z_{i, k}\right]=X^{\mu}\left(x_{i-1, k}, y_{i-1, k}, z_{i, k}\right)-X^{\mu}\left(x_{i-1, k}, y_{i-1, k}, z_{i-1, k}\right) .
\end{aligned}
$$

Lemma 2 In the case of $(j, k)$ for $\mu=1,2,3$ and $j=1,2, \ldots, k$ and $k=1,2, \ldots, n$, the following holds:

$$
\begin{aligned}
\left(X^{\mu}\right)_{j, k}= & \left(X^{\mu}\right)_{0,0}+\sum_{h=1}^{k} \frac{\Delta X^{\mu}\left[\Delta_{h}\left(x^{\gamma}\right)_{0, h}\right]}{\Delta_{h}\left(x^{\gamma}\right)_{0, h}} \Delta_{h}\left(x^{\gamma}\right)_{0, h} \\
& +\sum_{i=1}^{j} \frac{\Delta X^{\mu}\left[\Delta_{i}\left(x^{\gamma}\right)_{i, k}\right]}{\Delta_{i}\left(x^{\gamma}\right)_{i, k}} \Delta_{i}\left(x^{\gamma}\right)_{i, k}
\end{aligned}
$$

where the index is summed over $\gamma=1,2,3$. 
Proof Using (3.29) and (3.34), $\left(X^{\mu}\right)_{j, k}$ for $\mu=1,2,3$ and $j=1,2, \ldots, k$ and $k=1,2, \ldots, n$ is split into

$$
\left(X^{\mu}\right)_{j, k}=\left(X^{\mu}\right)_{0,0}+\sum_{h=1}^{k} \Delta_{h}\left(X^{\mu}\right)_{0, h}+\sum_{i=1}^{j} \Delta_{i}\left(X^{\mu}\right)_{i, k}
$$

1. Substituting (3.31), (3.32), and (3.33) into (3.29) for $\mu=1,2,3$ and $h=1,2, \ldots, k$, we obtain

$$
\begin{aligned}
\Delta_{h}\left(X^{\mu}\right)_{0, h} & =\Delta X^{\mu}\left[\Delta_{h} x_{0, h}\right]+\Delta X^{\mu}\left[\Delta_{h} y_{0, h}\right]+\Delta X^{\mu}\left[\Delta_{h} z_{0, h}\right] \\
& =\frac{\Delta X^{\mu}\left[\Delta_{h}\left(x^{\gamma}\right)_{0, h}\right]}{\Delta_{h}\left(x^{\gamma}\right)_{0, h}} \Delta_{h}\left(x^{\gamma}\right)_{0, h^{\prime}}
\end{aligned}
$$

where the index is summed over $\gamma=1,2,3$.

2. Substituting (3.36), (3.37), and (3.38) into (3.34) for $\mu=1,2,3$ and $i=1,2, \ldots, j$ and $k=1,2, \ldots, n$, we obtain

$$
\begin{aligned}
\Delta_{i}\left(X^{\mu}\right)_{i, k} & =\Delta X^{\mu}\left[\Delta_{i} x_{i, k}\right]+\Delta X^{\mu}\left[\Delta_{i} y_{i, k}\right]+\Delta X^{\mu}\left[\Delta_{i} z_{i, k}\right] \\
& =\frac{\Delta X^{\mu}\left[\Delta_{i}\left(x^{\gamma}\right)_{i, k}\right]}{\Delta_{i}\left(x^{\gamma}\right)_{i, k}} \Delta_{i}\left(x^{\gamma}\right)_{i, k}
\end{aligned}
$$

where the index is summed over $\gamma=1,2,3$.

Substituting (3.41) and (3.42) into (3.40), we obtain (3.39).

Lemma 3 In the case of $(k, j)$ for $\mu=1,2,3$ and $j=1,2, \ldots, k$ and $k=1,2, \ldots, n$, the following holds:

$$
\begin{aligned}
\left(X^{\mu}\right)_{k, j}= & \left(X^{\mu}\right)_{0,0}+\sum_{h=1}^{k} \frac{\Delta X^{\mu}\left[\Delta_{h}\left(x^{\gamma}\right)_{h, 0}\right]}{\Delta_{h}\left(x^{\gamma}\right)_{h, 0}} \Delta_{h}\left(x^{\gamma}\right)_{h, 0} \\
& +\sum_{i=1}^{j} \frac{\Delta X^{\mu}\left[\Delta_{i}\left(x^{\gamma}\right)_{k, i}\right]}{\Delta_{i}\left(x^{\gamma}\right)_{k, i}} \Delta_{i}\left(x^{\gamma}\right)_{k, i},
\end{aligned}
$$

where the index is summed over $\gamma=1,2,3$.

Proof In a similar manner as Lemma 2, we obtain (3.43).

Our triangular double and triple integrals and the divergence theorem in the 3D space are shown as follows.

Definition 4 The triangular area double integral in the $3 \mathrm{D}$ space $\iint_{\partial D} X^{\mu} d^{2} \sigma_{\mu}$ is defined as

$$
\iint_{\partial D} X^{\mu} d^{2} \sigma_{\mu}=\lim _{n \rightarrow \infty} \sum_{k=1}^{n} \sum_{j=1}^{k}\left[\left(X^{\mu}\right)_{j, k}\left(\Delta^{2} \sigma_{\mu}\right)_{j, k}+\left(X^{\mu}\right)_{k, j}\left(\Delta^{2} \sigma_{\mu}\right)_{k, j}\right]
$$

where $\partial D$ is the boundary of a domain, $d^{2} \sigma_{\mu}$ is the antisymmetric infinitesimal area element vector and the index is summed over $\mu=1,2,3$. 
Definition 5 The triangular triple integral for integrands of which are partial differentials in the 3D space $\iiint_{D} \frac{\partial X^{\prime \prime \mu}}{\partial x^{\prime \prime \gamma}} d x^{\prime \prime \gamma} d^{2} \sigma_{\mu}$ is defined as

$$
\begin{aligned}
& \iiint_{D} \frac{\partial X^{\prime \prime \mu}}{\partial x^{\prime \prime \gamma}} d x^{\prime \prime \gamma} d^{2} \sigma_{\mu} \\
&=\lim _{n \rightarrow \infty} \sum_{k=1}^{n} \sum_{j=1}^{k}\left\{\left[\sum_{h=1}^{k}\left(\frac{\Delta X^{\mu}\left[\Delta_{h}\left(x^{\gamma}\right)_{0, h}\right]}{\Delta_{h}\left(x^{\gamma}\right)_{0, h}} \Delta_{h}\left(x^{\gamma}\right)_{0, h}\right)\right.\right. \\
&\left.\quad+\sum_{i=1}^{j}\left(\frac{\Delta X^{\mu}\left[\Delta_{i}\left(x^{\gamma}\right)_{i, k}\right]}{\Delta_{i}\left(x^{\gamma}\right)_{i, k}} \Delta_{i}\left(x^{\gamma}\right)_{i, k}\right)\right]\left(\Delta^{2} \sigma_{\mu}\right)_{j, k} \\
&+\left[\sum_{h=1}^{k}\left(\frac{\Delta X^{\mu}\left[\Delta_{h}\left(x^{\gamma}\right)_{h, 0}\right]}{\Delta_{h}\left(x^{\gamma}\right)_{h, 0}} \Delta_{h}\left(x^{\gamma}\right)_{h, 0}\right)\right. \\
&\left.\left.\quad+\sum_{i=1}^{j}\left(\frac{\Delta X^{\mu}\left[\Delta_{i}\left(x^{\gamma}\right)_{k, i}\right]}{\Delta_{i}\left(x^{\gamma}\right)_{k, i}} \Delta_{i}\left(x^{\gamma}\right)_{k, i}\right)\right]\left(\Delta^{2} \sigma_{\mu}\right)_{k, j}\right\},
\end{aligned}
$$

where $D$ is a domain and the indices are summed over $\gamma, \mu=1,2,3$.

The following proposition is necessary for the condition (3.47) in Theorem 2.

Proposition 2 Denote constants as $C^{1}=C^{x}, C^{2}=C^{y}$, and $C^{3}=C^{z}$, then

$$
C^{\mu} \iint_{\partial D} d^{2} \sigma_{\mu}=C^{\mu} \lim _{n \rightarrow \infty} \sum_{k=1}^{n} \sum_{j=1}^{k}\left[\left(\Delta^{2} \sigma_{\mu}\right)_{j, k}+\left(\Delta^{2} \sigma_{\mu}\right)_{k, j}\right]
$$

holds, where the index is summed over $\mu=1,2,3$.

Proof The proof of this proposition is shown in The divergence theorem of a triangular integral [6].

The following is the revised version of the theorem shown in The divergence theorem of a triangular integral [6].

Theorem 2 (The divergence theorem of the triangular integral in the 3D space) Assume that $D$ is a domain and $\partial D$ is the boundary of the domain in the $3 D$ space, expressed in the Cartesian coordinates $(x, y, z) \in \mathbb{R}^{3}$. Let $X^{\mu}$ for $\mu=1,2,3$ be a set of partially differentiable functions with respect to $x^{\nu}$ for $v=1,2,3$ in $D$, where $X^{\mu}=X^{\mu}\left(x^{\nu}\right)$. In the case of a closed $2 D$ surface which satisfies

$$
C^{\mu} \iint_{\partial D} d^{2} \sigma_{\mu}=0
$$

the divergence theorem of the triangular integral in the $3 D$ space holds:

$$
\oiint_{\partial D} X^{\mu} d^{2} \sigma_{\mu}=\iiint_{D} \frac{\partial X^{\prime \prime \mu}}{\partial x^{\prime \prime \gamma}} d x^{\prime \prime \gamma} d^{2} \sigma_{\mu}
$$

where the indices are summed over $\gamma, \mu=1,2,3$ and $C^{\mu}$ are constants. 
Proof Combining (3.39) with (3.12) and combining (3.43) with (3.13) for $j=1,2, \ldots, k$ and $k=1,2, \ldots, n$, we obtain

$$
\begin{aligned}
& \sum_{k=1}^{n} \sum_{j=1}^{k}\left[\left(X^{\mu}\right)_{j, k}\left(\Delta^{2} \sigma_{\mu}\right)_{j, k}+\left(X^{\mu}\right)_{k, j}\left(\Delta^{2} \sigma_{\mu}\right)_{k, j}\right] \\
& =\sum_{k=1}^{n} \sum_{j=1}^{k}\left[\sum_{h=1}^{k}\left(\frac{\Delta X^{\mu}\left[\Delta_{h}\left(x^{\gamma}\right)_{0, h}\right]}{\Delta_{h}\left(x^{\gamma}\right)_{0, h}} \Delta_{h}\left(x^{\gamma}\right)_{0, h}\right)\right. \\
& \left.+\sum_{i=1}^{j}\left(\frac{\Delta X^{\mu}\left[\Delta_{i}\left(x^{\gamma}\right)_{i, k}\right]}{\Delta_{i}\left(x^{\gamma}\right)_{i, k}} \Delta_{i}\left(x^{\gamma}\right)_{i, k}\right)\right]\left(\Delta^{2} \sigma_{\mu}\right)_{j, k} \\
& +\sum_{k=1}^{n} \sum_{j=1}^{k}\left[\sum_{h=1}^{k}\left(\frac{\Delta X^{\mu}\left[\Delta_{h}\left(x^{\gamma}\right)_{h, 0}\right]}{\Delta_{h}\left(x^{\gamma}\right)_{h, 0}} \Delta_{h}\left(x^{\gamma}\right)_{h, 0}\right)\right. \\
& \left.\quad+\sum_{i=1}^{j}\left(\frac{\Delta X^{\mu}\left[\Delta_{i}\left(x^{\gamma}\right)_{k, i}\right]}{\Delta_{i}\left(x^{\gamma}\right)_{k, i}} \Delta_{i}\left(x^{\gamma}\right)_{k, i}\right)\right]\left(\Delta^{2} \sigma_{\mu}\right)_{k, j} \\
& \quad+\left(X^{\mu}\right)_{0,0} \sum_{k=1}^{n} \sum_{j=1}^{k}\left[\left(\Delta^{2} \sigma_{\mu}\right)_{j, k}+\left(\Delta^{2} \sigma_{\mu}\right)_{k, j}\right],
\end{aligned}
$$

where the indices are summed over $\gamma, \mu=1,2,3$. Using Proposition 2, (3.47) is rewritten as

$$
\left(X^{\mu}\right)_{0,0} \lim _{n \rightarrow \infty} \sum_{k=1}^{n} \sum_{j=1}^{k}\left[\left(\Delta^{2} \sigma_{\mu}\right)_{j, k}+\left(\Delta^{2} \sigma_{\mu}\right)_{k, j}\right]=0
$$

where the index is summed over $\mu=1,2,3$. The limit at infinity $n \rightarrow \infty$ of (3.49) is expressed as (3.48) by Definitions 4 and 5 under the condition of a closed surface (3.50).

The triangular triple integral for a 3-variable function $q=q(x, y, z)$ in the 3D space by the infinitesimal volume element $d^{3} V$ is given by

$$
\iiint_{D} q d^{3} V=\frac{1}{3} \iiint_{D} q d x^{\prime \prime \gamma} d^{2} \sigma_{\gamma}
$$

where the index is summed over $\gamma=1,2,3$.

The calculation process of a triangular triple integral in the 3D space is precisely defined as follows.

Let $q=q(x, y, z)$ be a piecewise smooth function in the 3D plane, expressed in the Cartesian coordinates $(x, y, z) \in \mathbb{R}^{3}$.

Definition 6 The triangular triple integral for a 3-variable function $q=q(x, y, z)$ in the 3D space $\frac{1}{3} \iiint_{D} q d x^{\prime \prime \gamma} d^{2} \sigma_{\gamma}$ is defined as

$$
\begin{aligned}
& \frac{1}{3} \iiint_{D} q d x^{\prime \prime \gamma} d^{2} \sigma_{\gamma} \\
& \quad=\frac{1}{3} \lim _{h \rightarrow \infty} \sum_{k=1}^{n} \sum_{j=1}^{k}\left\{\left[\sum_{h=1}^{k} q\left(\left(x^{\alpha}\right)_{j, k}\left(x^{\beta}\right)_{j, k}\left(x^{\gamma}\right)_{0, h}\right) \Delta_{h}\left(x^{\gamma}\right)_{0, h}\right.\right.
\end{aligned}
$$




$$
\begin{aligned}
& \left.+\sum_{i=1}^{j} q\left(\left(x^{\alpha}\right)_{j, k},\left(x^{\beta}\right)_{j, k},\left(x^{\gamma}\right)_{i, k}\right) \Delta_{i}\left(x^{\gamma}\right)_{i, k}\right]\left(\Delta^{2} \sigma_{\gamma}\right)_{j, k} \\
& +\left[\sum_{h=1}^{k} q\left(\left(x^{\alpha}\right)_{k, j^{\prime}}\left(x^{\beta}\right)_{k, j^{\prime}}\left(x^{\gamma}\right)_{h, 0}\right) \Delta_{h}\left(x^{\gamma}\right)_{h, 0}\right. \\
& \left.\left.+\sum_{i=1}^{j} q\left(\left(x^{\alpha}\right)_{k, j^{\prime}}\left(x^{\beta}\right)_{k, j^{\prime}}\left(x^{\gamma}\right)_{k, i}\right) \Delta_{i}\left(x^{\gamma}\right)_{k, i}\right]\left(\Delta^{2} \sigma_{\gamma}\right)_{k, j}\right\}
\end{aligned}
$$

for $\alpha \neq \beta, \beta \neq \gamma, \gamma \neq \alpha$, and $\alpha, \beta=1,2,3$ as the indices of variable of function $q=q(x, y, z)$, where $D$ is a domain and the index is summed over $\gamma=1,2,3$.

The revised corollary shown below derived from Theorem 2 is the 3D version of Corollary 1.

Corollary 2 (A corollary of Theorem 2) Assume that $D$ is a domain and $\partial D$ is the boundary of the domain in the $3 D$ space, expressed in the Cartesian coordinates $(x, y, z) \in \mathbb{R}^{3}$. Let $X^{\mu}$ for $\mu=1,2,3$ be a set of partially differentiable functions with respect to $x^{\nu}$ for $v=1,2,3$ in $D$, where $X^{\mu}=X^{\mu}\left(x^{\nu}\right)$. In the case of

$$
\oiint_{\partial D} X^{\mu} d^{2} \sigma_{\mu}=\iiint_{D} q d^{3} V
$$

where $q=q(x, y, z)$ is a 3-variable function and the index is summed over $\mu=1,2,3$, the following holds:

$$
\frac{\partial X^{\mu}}{\partial x^{\mu}}=q
$$

Proof Substituting (3.48) and (3.51) into (3.53), it is rewritten as

$$
\iiint_{D} \frac{\partial X^{\prime \prime \mu}}{\partial x^{\prime \prime \gamma}} d x^{\prime \prime \gamma} d^{2} \sigma_{\mu}=\frac{1}{3} \iiint_{D} q d x^{\prime \prime \gamma} d^{2} \sigma_{\gamma}
$$

where the indices are summed over $\gamma, \mu=1,2,3$. Substituting (3.45) and (3.52) into (3.55), it is expressed as

$$
\begin{aligned}
\lim _{n \rightarrow \infty} \sum_{k=1}^{n} \sum_{j=1}^{k}\left\{\left[\sum_{h=1}^{k} \frac{\Delta X^{\mu}\left[\Delta_{h}\left(x^{\gamma}\right)_{0, h}\right]}{\Delta_{h}\left(x^{\gamma}\right)_{0, h}} \Delta_{h}\left(x^{\gamma}\right)_{0, h}\right.\right. \\
\left.\quad+\sum_{i=1}^{j} \frac{\Delta X^{\mu}\left[\Delta_{i}\left(x^{\gamma}\right)_{i, k}\right]}{\Delta_{i}\left(x^{\gamma}\right)_{i, k}} \Delta_{i}\left(x^{\gamma}\right)_{i, k}\right] \frac{1}{2} \varepsilon_{\alpha \beta \mu}\left(\Delta^{2} \sigma^{\alpha \beta}\right)_{j, k} \\
\quad+\left[\sum_{h=1}^{k} \frac{\Delta X^{\mu}\left[\Delta_{h}\left(x^{\gamma}\right)_{h, 0}\right]}{\Delta_{h}\left(x^{\gamma}\right)_{h, 0}} \Delta_{h}\left(x^{\gamma}\right)_{h, 0}\right. \\
\left.\left.\quad+\sum_{i=1}^{j} \frac{\Delta X^{\mu}\left[\Delta_{i}\left(x^{\gamma}\right)_{k, i}\right]}{\Delta_{i}\left(x^{\gamma}\right)_{k, i}} \Delta_{i}\left(x^{\gamma}\right)_{k, i}\right] \frac{1}{2} \varepsilon_{\alpha \beta \mu}\left(\Delta^{2} \sigma^{\alpha \beta}\right)_{k, j}\right\} \\
=\frac{1}{3} \lim _{n \rightarrow \infty} \sum_{k=1}^{n} \sum_{j=1}^{k}\left\{\left[\sum_{h=1}^{k} q\left(\left(x^{\alpha}\right)_{j, k},\left(x^{\beta}\right)_{j, k},\left(x^{\gamma}\right)_{0, h}\right) \Delta_{h}\left(x^{\gamma}\right)_{0, h}\right.\right.
\end{aligned}
$$




$$
\begin{aligned}
& \left.+\sum_{i=1}^{j} q\left(\left(x^{\alpha}\right)_{j, k},\left(x^{\beta}\right)_{j, k},\left(x^{\gamma}\right)_{i, k}\right) \Delta_{i}\left(x^{\gamma}\right)_{i, k}\right] \frac{1}{2} \varepsilon_{\alpha \beta \gamma}\left(\Delta^{2} \sigma^{\alpha \beta}\right)_{j, k} \\
& +\left[\sum_{h=1}^{k} q\left(\left(x^{\alpha}\right)_{k, j},\left(x^{\beta}\right)_{k, j},\left(x^{\gamma}\right)_{h, 0}\right) \Delta_{h}\left(x^{\gamma}\right)_{h, 0}\right. \\
& \left.+\sum_{i=1}^{j} q\left(\left(x^{\alpha}\right)_{k, j},\left(x^{\beta}\right)_{k, j^{\prime}},\left(x^{\gamma}\right)_{k, i} \Delta_{i}\left(x^{\gamma}\right)_{k, i}\right] \frac{1}{2} \varepsilon_{\alpha \beta \gamma}\left(\Delta^{2} \sigma^{\alpha \beta}\right)_{k, j}\right\},
\end{aligned}
$$

where the indices are summed over $\alpha, \beta, \gamma, \mu=1,2,3$. In order for (3.56) to hold for any value of integral variables, the following $2 \times 2 !=4$ kinds of formulae in two categories are required.

1. The formulae for $\alpha, \beta, \gamma=1,2,3$ and $h=1,2, \ldots, k$,

$$
\begin{aligned}
\lim _{\Delta_{h}\left(x^{\gamma}\right)_{0, h} \rightarrow 0} \frac{\Delta X^{\mu}\left[\Delta_{h}\left(x^{\gamma}\right)_{0, h}\right]}{\Delta_{h}\left(x^{\gamma}\right)_{0, h}} \frac{1}{2} \varepsilon_{\alpha \beta \mu} & =q\left(\left(x^{\alpha}\right)_{j, k},\left(x^{\beta}\right)_{j, k^{\prime}},\left(x^{\gamma}\right)_{0, h}\right) \frac{1}{3 !} \varepsilon_{\alpha \beta \gamma}, \\
\lim _{\Delta_{h}\left(x^{\gamma}\right)_{h, 0} \rightarrow 0} \frac{\Delta X^{\mu}\left[\Delta_{h}\left(x^{\gamma}\right)_{h, 0}\right]}{\Delta_{h}\left(x^{\gamma}\right)_{h, 0}} \frac{1}{2} \varepsilon_{\alpha \beta \mu} & =q\left(\left(x^{\alpha}\right)_{k, j^{\prime}}\left(x^{\beta}\right)_{k, j^{\prime}}\left(x^{\gamma}\right)_{h, 0}\right) \frac{1}{3 !} \varepsilon_{\alpha \beta \gamma},
\end{aligned}
$$

where the index is summed over $\mu=1,2,3$.

2. The formulae for $\alpha, \beta, \gamma=1,2,3$ and $i=1,2, \ldots, j$ and $k=1,2, \ldots, n$,

$$
\begin{array}{r}
\lim _{\Delta_{i}\left(x^{\gamma}\right)_{i, k} \rightarrow 0} \frac{\Delta X^{\mu}\left[\Delta_{i}\left(x^{\gamma}\right)_{i, k}\right]}{\Delta_{i}\left(x^{\gamma}\right)_{i, k}} \frac{1}{2} \varepsilon_{\alpha \beta \mu}=q\left(\left(x^{\alpha}\right)_{j, k},\left(x^{\beta}\right)_{j, k^{\prime}}\left(x^{\gamma}\right)_{i, k}\right) \frac{1}{3 !} \varepsilon_{\alpha \beta \gamma}, \\
\lim _{\Delta_{i}\left(x^{\gamma}\right)_{k, i} \rightarrow 0} \frac{\Delta X^{\mu}\left[\Delta_{i}\left(x^{\gamma}\right)_{k, i}\right]}{\Delta_{i}\left(x^{\gamma}\right)_{k, i}} \frac{1}{2} \varepsilon_{\alpha \beta \mu}=q\left(\left(x^{\alpha}\right)_{k, j^{\prime}}\left(x^{\beta}\right)_{k, j^{\prime}}\left(x^{\gamma}\right)_{k, i}\right) \frac{1}{3 !} \varepsilon_{\alpha \beta \gamma},
\end{array}
$$

where the index is summed over $\mu=1,2,3$.

In the 3D space, $\frac{1}{2} \varepsilon_{\alpha \beta \mu} \varepsilon^{\alpha \beta \gamma}=\delta_{\mu}^{\gamma}$ holds for $\gamma, \mu=1,2,3$, where the indices are summed over $\alpha, \beta=1,2,3$. We therefore obtain $\frac{1}{3 !} \varepsilon_{\alpha \beta \gamma} \varepsilon^{\alpha \beta \gamma}=1$, where the indices are summed over $\alpha, \beta, \gamma=1,2,3$. Multiplying by $\varepsilon^{\alpha \beta \gamma}$ both sides of the four kinds of (3.57), (3.58), (3.59), and (3.60), they are reduced to the differential equation (3.54).

\subsection{Component representation and an example of it for a 3-variable function}

The left-hand side of (3.44) is expressed as

$$
\iint_{\partial D} X^{\mu} d^{2} \sigma_{\mu}=\iint_{\partial D} X d^{2} \sigma_{x}+\iint_{\partial D} Y d^{2} \sigma_{y}+\iint_{\partial D} Z d^{2} \sigma_{z}
$$

where the index is summed over $\mu=1,2,3$. Using (3.44) in Definition 4, each component of (3.61) is

$$
\begin{aligned}
& \iint_{\partial D} X d^{2} \sigma_{x}=\lim _{n \rightarrow \infty} \sum_{k=1}^{n} \sum_{j=1}^{k}\left[X_{j, k}\left(\Delta^{2} \sigma_{x}\right)_{j, k}+X_{k, j}\left(\Delta^{2} \sigma_{x}\right)_{k, j}\right] \\
& \iint_{\partial D} Y d^{2} \sigma_{y}=\lim _{n \rightarrow \infty} \sum_{k=1}^{n} \sum_{j=1}^{k}\left[Y_{j, k}\left(\Delta^{2} \sigma_{y}\right)_{j, k}+Y_{k, j}\left(\Delta^{2} \sigma_{y}\right)_{k, j}\right] \\
& \iint_{\partial D} Z d^{2} \sigma_{z}=\lim _{n \rightarrow \infty} \sum_{k=1}^{n} \sum_{j=1}^{k}\left[Z_{j, k}\left(\Delta^{2} \sigma_{z}\right)_{j, k}+Z_{k, j}\left(\Delta^{2} \sigma_{z}\right)_{k, j}\right]
\end{aligned}
$$


In component representation, (3.51) is expressed as

$$
\iiint_{D} q d^{3} V=\frac{1}{3} \iiint_{D} q d x^{\prime \prime} d^{2} \sigma_{x}+\frac{1}{3} \iiint_{D} q d y^{\prime \prime} d^{2} \sigma_{y}+\frac{1}{3} \iiint_{D} q d z^{\prime \prime} d^{2} \sigma_{z} .
$$

Using (3.52) in Definition 6, each component of (3.65) is

$$
\begin{aligned}
& \frac{1}{3} \iiint_{D} q d x^{\prime \prime} d^{2} \sigma_{x} \\
& =\frac{1}{3} \lim _{n \rightarrow \infty} \sum_{k=1}^{n} \sum_{j=1}^{k}\left\{\left[\sum_{h=1}^{k} q\left(x_{0, h}, y_{j, k}, z_{j, k}\right)_{h} x_{0, h}+\sum_{i=1}^{j} q\left(x_{i, k}, y_{j, k}, z_{j, k}\right)_{i} x_{i, k}\right]\left(\Delta^{2} \sigma_{x}\right)_{j, k}\right. \\
& \left.+\left[\sum_{h=1}^{k} q\left(x_{h, 0}, y_{k, j}, z_{k, j}\right) \Delta_{h} x_{h, 0}+\sum_{i=1}^{j} q\left(x_{k, i}, y_{k, j}, z_{k, j}\right) \Delta_{i} x_{k, i}\right]\left(\Delta^{2} \sigma_{x}\right)_{k, j}\right\}, \\
& \frac{1}{3} \iint_{D} q d y^{\prime \prime} d^{2} \sigma_{y} \\
& =\frac{1}{3} \lim _{n \rightarrow \infty} \sum_{k=1}^{n} \sum_{j=1}^{k}\left\{\left[\sum_{h=1}^{k} q\left(x_{j, k}, y_{0, h}, z_{j, k}\right) \Delta_{h} y_{0, h}+\sum_{i=1}^{j} q\left(x_{j, k}, y_{i, k}, z_{j, k}\right) \Delta_{i} y_{i, k}\right]\left(\Delta^{2} \sigma_{y}\right)_{j, k}\right. \\
& \left.\quad+\left[\sum_{h=1}^{k} q\left(x_{k, j}, y_{h, 0}, z_{k, j}\right) \Delta_{h} y_{h, 0}+\sum_{i=1}^{j} q\left(x_{k, j}, y_{k, i}, z_{k, j}\right) \Delta_{i} y_{k, i}\right]\left(\Delta^{2} \sigma_{y}\right)_{k, j}\right\}, \\
& \frac{1}{3} \iint_{D} q d z^{\prime \prime} d^{2} \sigma_{z} \\
& \left.\quad \frac{1}{3} \lim _{n \rightarrow \infty} \sum_{k=1}^{n} \sum_{j=1}^{k}\left\{\left[\sum_{h=1}^{k} q\left(x_{j, k}, y_{j, k}, z_{0, h}\right) \Delta_{h} z_{0, h}+\sum_{i=1}^{j} q\left(x_{j, k}, y_{j, k}, z_{i, k}\right) \Delta_{i} z_{i, k}\right]\left(\Delta_{h, 0}\right) \Delta_{h} z_{h, 0}+\sum_{i=1}^{j} q\left(x_{k, j}, y_{k, j}, z_{k, i}\right) \Delta_{i, k} z_{k, i}\right]\left(\Delta^{2} \sigma_{z}\right)_{k, j}\right\} .
\end{aligned}
$$

In Corollary 2, (3.53) is expressed as

$$
\oiint_{\partial D}\left(X d^{2} \sigma_{x}+Y d^{2} \sigma_{y}+Z d^{2} \sigma_{z}\right)=\iiint_{D} q d^{3} V
$$

and (3.54) is expressed as

$$
\frac{\partial X}{\partial x}+\frac{\partial Y}{\partial y}+\frac{\partial Z}{\partial z}=q
$$

We show an example of Corollary 2 in the following.

Example 2 In the case of

$$
\begin{aligned}
& X(x, y, z)=\frac{1}{2} x\left(y^{2}+z^{2}\right), \quad Y(x, y, z)=\frac{1}{2} y\left(z^{2}+x^{2}\right), \\
& Z(x, y, z)=\frac{1}{2} z\left(x^{2}+y^{2}\right) .
\end{aligned}
$$


Substituting (3.71) into (3.70), we obtain

$$
q(x, y, z)=x^{2}+y^{2}+z^{2}
$$

The boundary of the domain is a sphere (3.22).

Since $X_{0,0}=0, Y_{0,0}=0$, and $Z_{0,0}=0$, (3.71) satisfies the condition of a closed surface (3.50).

1. The value of the left-hand side of (3.69) is

$$
\oiint_{\partial D}\left(X d^{2} \sigma_{x}+Y d^{2} \sigma_{y}+Z d^{2} \sigma_{z}\right)=\frac{4}{5} \pi a^{5} .
$$

See (B.3), (B.6), and (B.9) in Appendix 2 for calculations in detail.

The value of the right-hand side of (3.69) is

$$
\begin{aligned}
\iiint_{D} q d^{3} V & =\frac{1}{3} \iiint q d x^{\prime \prime} d^{2} \sigma_{x}+\frac{1}{3} \iiint q d y^{\prime \prime} d^{2} \sigma_{y}+\frac{1}{3} \iiint q d z^{\prime \prime} d^{2} \sigma_{z} \\
& =\frac{4}{5} \pi a^{5} .
\end{aligned}
$$

See (B.14), (B.19), and (B.24) in Appendix 2 for calculations in detail.

We thus see the coincidence of the value of (3.73) and that of (3.74).

We consider the following approximation formula of (3.69) for $1 \ll n<\infty$ :

$$
\begin{aligned}
& \sum_{k=1}^{n} \sum_{j=1}^{k}\left[X\left(x_{j, k}, y_{j, k}, z_{j, k}\right)\left(\Delta^{2} \sigma_{x}\right)_{j, k}+X\left(x_{k, j}, y_{k, j}, z_{k, j}\right)\left(\Delta^{2} \sigma_{x}\right)_{k, j}+Y\left(x_{j, k}, y_{j, k}, z_{j, k}\right)\left(\Delta^{2} \sigma_{y}\right)_{j, k}\right. \\
& \left.+Y\left(x_{k, j}, y_{k, j}, z_{k, j}\right)\left(\Delta^{2} \sigma_{y}\right)_{k, j}+Z\left(x_{j, k}, y_{j, k}, z_{j, k}\right)\left(\Delta^{2} \sigma_{z}\right)_{j, k}+Z\left(x_{k, j}, y_{k, j}, z_{k, j}\right)\left(\Delta^{2} \sigma_{z}\right)_{k, j}\right] \\
& \simeq \frac{1}{3} \sum_{k=1}^{n} \sum_{j=1}^{k}\left[\sum_{h=1}^{k} q\left(x_{0, h}, y_{j, k}, z_{j, k}\right) \Delta_{h} x_{0, h}+\sum_{i=1}^{j} q\left(x_{i, k}, y_{j, k}, z_{j, k}\right) \Delta_{i} x_{i, k}\right]\left(\Delta^{2} \sigma_{x}\right)_{j, k} \\
& +\frac{1}{3} \sum_{k=1}^{n} \sum_{j=1}^{k}\left[\sum_{h=1}^{k} q\left(x_{h, 0}, y_{k, j}, z_{k, j}\right) \Delta_{h} x_{h, 0}+\sum_{i=1}^{j} q\left(x_{k, i}, y_{k, j}, z_{k, j}\right) \Delta_{i} x_{k, i}\right]\left(\Delta^{2} \sigma_{x}\right)_{k, j} \\
& +\frac{1}{3} \sum_{k=1}^{n} \sum_{j=1}^{k}\left[\sum_{h=1}^{k} q\left(x_{j, k}, y_{0, h}, z_{j, k}\right) \Delta_{h} y_{0, h}+\sum_{i=1}^{j} q\left(x_{j, k}, y_{i, k}, z_{j, k}\right) \Delta_{i} y_{i, k}\right]\left(\Delta^{2} \sigma_{y}\right)_{j, k} \\
& +\frac{1}{3} \sum_{k=1}^{n} \sum_{j=1}^{k}\left[\sum_{h=1}^{k} q\left(x_{k, j}, y_{h, 0}, z_{k, j}\right) \Delta_{h} y_{h, 0}+\sum_{i=1}^{j} q\left(x_{k, j}, y_{k, i}, z_{k, j}\right) \Delta_{i} y_{k, i}\right]\left(\Delta^{2} \sigma_{y}\right)_{k, j} \\
& +\frac{1}{3} \sum_{k=1}^{n} \sum_{j=1}^{k}\left[\sum_{h=1}^{k} q\left(x_{j, k}, y_{j, k}, z_{0, h}\right) \Delta_{h} z_{0, h}+\sum_{i=1}^{j} q\left(x_{j, k}, y_{j, k}, z_{i, k}\right) \Delta_{i} z_{i, k}\right]\left(\Delta^{2} \sigma_{z}\right)_{j, k} \\
& +\frac{1}{3} \sum_{k=1}^{n} \sum_{j=1}^{k}\left[\sum_{h=1}^{k} q\left(x_{k, j}, y_{k, j}, z_{h, 0}\right) \Delta_{h} z_{h, 0}\right. \\
& \left.+\sum_{i=1}^{j} q\left(x_{k, j}, y_{k, j}, z_{k, i}\right) \Delta_{i} z_{k, i}\right]\left(\Delta^{2} \sigma_{z}\right)_{k, j}
\end{aligned}
$$


In the case of $n \rightarrow \infty$, (3.75) coincides with (3.69). It is verified by (3.73) and (3.74) in Example 2. An approximation formula, see (3.69), for Example 2 is

$$
\begin{aligned}
\frac{1}{2} \sum_{k=1}^{n} & \sum_{j=1}^{k} x_{j, k}\left(y_{j, k}^{2}+z_{j, k}^{2}\right)\left(\Delta^{2} \sigma_{x}\right)_{j, k}+\frac{1}{2} \sum_{k=1}^{n} \sum_{j=1}^{k} x_{k, j}\left(y_{k, j}^{2}+z_{k, j}^{2}\right)\left(\Delta^{2} \sigma_{x}\right)_{k, j} \\
& +\frac{1}{2} \sum_{k=1}^{n} \sum_{j=1}^{k} y_{j, k}\left(z_{j, k}^{2}+x_{j, k}^{2}\right)\left(\Delta^{2} \sigma_{y}\right)_{j, k}+\frac{1}{2} \sum_{k=1}^{n} \sum_{j=1}^{k} y_{k, j}\left(z_{k, j}^{2}+x_{k, j}^{2}\right)\left(\Delta^{2} \sigma_{y}\right)_{k, j} \\
& +\frac{1}{2} \sum_{k=1}^{n} \sum_{j=1}^{k} z_{j, k}\left(x_{j, k}^{2}+y_{j, k}^{2}\right)\left(\Delta^{2} \sigma_{z}\right)_{j, k}+\frac{1}{2} \sum_{k=1}^{n} \sum_{j=1}^{k} z_{k, j}\left(x_{k, j}^{2}+y_{k, j}^{2}\right)\left(\Delta^{2} \sigma_{z}\right)_{k, j} \\
\simeq & \frac{1}{3} \sum_{k=1}^{n} \sum_{j=1}^{k}\left[\sum_{h=1}^{k}\left(x_{0, h}^{2}+y_{j, k}^{2}+z_{j, k}^{2}\right) \Delta_{h} x_{0, h}+\sum_{i=1}^{j}\left(x_{i, k}^{2}+y_{j, k}^{2}+z_{j, k}^{2}\right) \Delta_{i} x_{i, k}\right]\left(\Delta^{2} \sigma_{x}\right)_{j, k} \\
& +\frac{1}{3} \sum_{k=1}^{n} \sum_{j=1}^{k}\left[\sum_{h=1}^{k}\left(x_{h, 0}^{2}+y_{k, j}^{2}+z_{k, j}^{2}\right) \Delta_{h} x_{h, 0}+\sum_{i=1}^{j}\left(x_{k, i}^{2}+y_{k, j}^{2}+z_{k, j}^{2}\right) \Delta_{i} x_{k, i}\right]\left(\Delta^{2} \sigma_{x}\right)_{k, j} \\
& +\frac{1}{3} \sum_{k=1}^{n} \sum_{j=1}^{k}\left[\sum_{h=1}^{k}\left(x_{j, k}^{2}+y_{0, h}^{2}+z_{j, k}^{2}\right) \Delta_{h} y_{0, h}+\sum_{i=1}^{j}\left(x_{j, k}^{2}+y_{i, k}^{2}+z_{j, k}^{2}\right) \Delta_{i} y_{i, k}\right]\left(\Delta^{2} \sigma_{y}\right)_{j, k} \\
& +\frac{1}{3} \sum_{k=1}^{n} \sum_{j=1}^{k}\left[\sum_{h=1}^{k}\left(x_{k, j}^{2}+y_{h, 0}^{2}+z_{k, j}^{2}\right) \Delta_{h} y_{h, 0}+\sum_{i=1}^{j}\left(x_{k, j}^{2}+y_{k, i}^{2}+z_{k, j}^{2}\right) \Delta_{i} y_{k, i}\right]\left(\Delta^{2} \sigma_{y}\right)_{k, j} \\
& +\frac{1}{3} \sum_{k=1}^{n} \sum_{j=1}^{k}\left[\sum_{h=1}^{k}\left(x_{j, k}^{2}+y_{j, k}^{2}+z_{0, h}^{2}\right) \Delta_{h} z_{0, h}+\sum_{i=1}^{j}\left(x_{j, k}^{2}+y_{j, k}^{2}+z_{i, k}^{2}\right) \Delta_{i} z_{i, k}\right]\left(\Delta^{2} \sigma_{z}\right)_{j, k} \\
& +\frac{1}{3} \sum_{k=1}^{n} \sum_{j=1}^{k}\left[\sum_{h=1}^{k}\left(x_{k, j}^{2}+y_{k, j}^{2}+z_{h, 0}^{2}\right) \Delta_{h} z_{h, 0}\right. \\
& \left.\left.\sum_{k, j}^{2}+z_{k, i}^{2}\right) \Delta_{i} z_{k, i}\right]\left(\Delta^{2} \sigma_{z}\right)_{k, j} \\
&
\end{aligned}
$$

The left- and the right-hand sides of (3.76), respectively expressed as $L$ and $R$, are shown in Table 2 and plotted in Figure 2, where $a=1$.

\section{Triangular integral in the 4D time-space}

Six kinds of combined and antisymmetric finite hyper-surface element vectors in the 4D time-space are reviewed in Section 4.1. The triangular quadruple integral for a 4-variable function is shown in Section 4.2. In the following, the Cartesian coordinates are denoted $x^{0}=t, x^{1}=x, x^{2}=y$, and $x^{3}=z$.

\subsection{Six kinds of finite hyper-surface element vectors in the 4D time-space}

For the triangular quadruple integral, the following increments of a triple sequence of points in the 4D time-space are introduced.

1. The increments of a triple sequence of points at $(j, k, l)$ are denoted as follows:

$$
\Delta_{j}\left(x^{\alpha}\right)_{j, k, l} \equiv\left(x^{\alpha}\right)_{j, k, l}-\left(x^{\alpha}\right)_{j-1, k, l}
$$


Table 2 Approximate values of Example 2

\begin{tabular}{llll}
\hline $\boldsymbol{n}$ & $\boldsymbol{L}$ & $\boldsymbol{R}$ & $\boldsymbol{R}-\boldsymbol{L}$ \\
\hline 2 & 0 & 0 & 0 \\
4 & $1.06694173 \ldots$ & $2.18060515 \ldots$ & $+1.11366341 \ldots$ \\
8 & $2.40955699 \ldots$ & $2.64197558 \ldots$ & $+0.23241859 \ldots$ \\
16 & $2.56697581 \ldots$ & $2.62093273 \ldots$ & $+0.05395691 \ldots$ \\
32 & $2.56645589 \ldots$ & $2.57577633 \ldots$ & $+0.00932043 \ldots$ \\
64 & $2.54629327 \ldots$ & $2.54651836 \ldots$ & $+0.00022508 \ldots$ \\
128 & $2.53136275 \ldots$ & $2.53038066 \ldots$ & $-0.00098209 \ldots$ \\
256 & $2.52270918 \ldots$ & $2.52194728 \ldots$ & $-0.00076189 \ldots$ \\
512 & $2.51808880 \ldots$ & $2.51764056 \ldots$ & $-0.00044824 \ldots$ \\
1,024 & $2.51570568 \ldots$ & $2.51546479 \ldots$ & $-0.00024088 \ldots$ \\
2,048 & $2.51449594 \ldots$ & $2.51437132 \ldots$ & $-0.00012462 \ldots$ \\
4,096 & $2.51388654 \ldots$ & $2.51382318 \ldots$ & $-0.00006335 \ldots$ \\
8,192 & $2.51358071 \ldots$ & $2.51354877 \ldots$ & $-0.00003194 \ldots$ \\
16,384 & $2.51342750 \ldots$ & $2.51341147 \ldots$ & $-0.00001602 \ldots$ \\
32,768 & $2.51335080 \ldots$ & $2.51334280 \ldots$ & $-0.00000799 \ldots$ \\
$\vdots$ & $\vdots$ & $\vdots$ & $\vdots$ \\
$\infty$ & $2.51327412 \ldots$ & $2.51327412 \ldots$ & \pm 0 \\
\hline
\end{tabular}

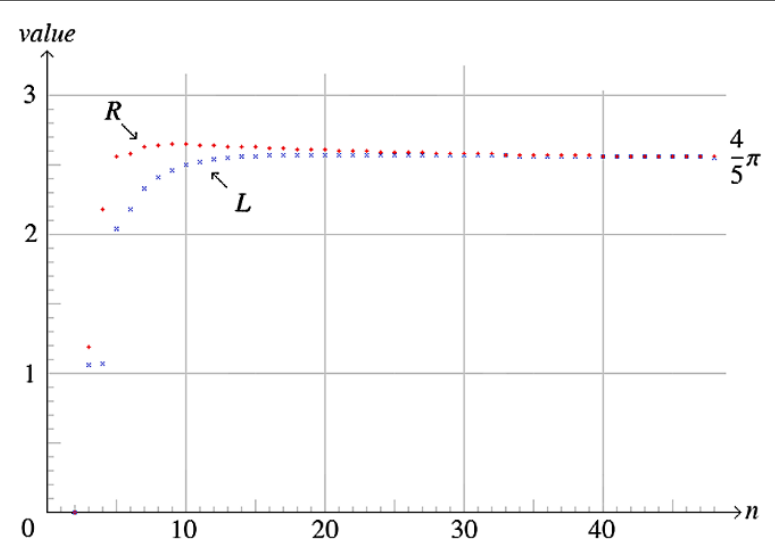

Figure 2 Approximate values of Example 2.

$$
\begin{aligned}
& \Delta_{k}\left(x^{\beta}\right)_{j-1, k, l} \equiv\left(x^{\beta}\right)_{j-1, k, l}-\left(x^{\beta}\right)_{j-1, k-1, l} \\
& \Delta_{l}\left(x^{\gamma}\right)_{j-1, k-1, l} \equiv\left(x^{\gamma}\right)_{j-1, k-1, l}-\left(x^{\gamma}\right)_{j-1, k-1, l-1}, \\
& \Delta_{k}\left(x^{\alpha}\right)_{j, k, l} \equiv\left(x^{\alpha}\right)_{j, k, l}-\left(x^{\alpha}\right)_{j, k-1, l}, \\
& \Delta_{l}\left(x^{\beta}\right)_{j, k-1, l} \equiv\left(x^{\beta}\right)_{j, k-1, l}-\left(x^{\beta}\right)_{j, k-1, l-1} \\
& \Delta_{j}\left(x^{\gamma}\right)_{j, k-1, l-1} \equiv\left(x^{\gamma}\right)_{j, k-1, l-1}-\left(x^{\gamma}\right)_{j-1, k-1, l-1}, \\
& \Delta_{l}\left(x^{\alpha}\right)_{j, k, l} \equiv\left(x^{\alpha}\right)_{j, k, l}-\left(x^{\alpha}\right)_{j, k, l-1} \\
& \Delta_{j}\left(x^{\beta}\right)_{j, k, l-1} \equiv\left(x^{\beta}\right)_{j, k, l-1}-\left(x^{\beta}\right)_{j-1, k, l-1} \\
& \Delta_{k}\left(x^{\gamma}\right)_{j-1, k, l-1} \equiv\left(x^{\gamma}\right)_{j-1, k, l-1}-\left(x^{\gamma}\right)_{j-1, k-1, l-1}
\end{aligned}
$$

for $\alpha, \beta, \gamma=0,1,2,3$ and $l=1,2, \ldots, j$ and $j=1,2, \ldots, k$ and $k=1,2, \ldots, n$. 
The first combined finite hyper-surface element vector $\left(\Delta^{3} V^{\alpha \beta \gamma}\right)_{j, k, l}$ for $\alpha, \beta, \gamma=0,1,2,3$ and $l=1,2, \ldots, j$ and $j=1,2, \ldots, k$ and $k=1,2, \ldots, n$ is introduced as

$$
\begin{aligned}
\left(\Delta^{3} V^{\alpha \beta \gamma}\right)_{j, k, l}= & -\Delta_{j}\left(x^{\alpha}\right)_{j, k, l} \Delta_{k}\left(x^{\beta}\right)_{j-1, k, l} \Delta_{l}\left(x^{\gamma}\right)_{j-1, k-1, l} \\
& -\Delta_{k}\left(x^{\alpha}\right)_{j, k, l} \Delta_{l}\left(x^{\beta}\right)_{j, k-1, l} \Delta_{j}\left(x^{\gamma}\right)_{j, k-1, l-1} \\
& -\Delta_{l}\left(x^{\alpha}\right)_{j, k, l} \Delta_{j}\left(x^{\beta}\right)_{j, k, l-1} \Delta_{k}\left(x^{\gamma}\right)_{j-1, k, l-1} .
\end{aligned}
$$

2. The increments of a triple sequence of points at $(j, l, k)$ are denoted as follows:

$$
\begin{aligned}
& \Delta_{j}\left(x^{\alpha}\right)_{j, l, k} \equiv\left(x^{\alpha}\right)_{j, l, k}-\left(x^{\alpha}\right)_{j-1, l, k} \\
& \Delta_{l}\left(x^{\beta}\right)_{j-1, l, k} \equiv\left(x^{\beta}\right)_{j-1, l, k}-\left(x^{\beta}\right)_{j-1, l-1, k} \\
& \Delta_{k}\left(x^{\gamma}\right)_{j-1, l-1, k} \equiv\left(x^{\gamma}\right)_{j-1, l-1, k}-\left(x^{\gamma}\right)_{j-1, l-1, k-1}, \\
& \Delta_{l}\left(x^{\alpha}\right)_{j, l, k} \equiv\left(x^{\alpha}\right)_{j, l, k}-\left(x^{\alpha}\right)_{j, l-1, k} \\
& \Delta_{k}\left(x^{\beta}\right)_{j, l-1, k} \equiv\left(x^{\beta}\right)_{j, l-1, k}-\left(x^{\beta}\right)_{j, l-1, k-1}, \\
& \Delta_{j}\left(x^{\gamma}\right)_{j, l-1, k-1} \equiv\left(x^{\gamma}\right)_{j, l-1, k-1}-\left(x^{\gamma}\right)_{j-1, l-1, k-1} \\
& \Delta_{k}\left(x^{\alpha}\right)_{j, l, k} \equiv\left(x^{\alpha}\right)_{j, l, k}-\left(x^{\alpha}\right)_{j, l, k-1} \\
& \Delta_{j}\left(x^{\beta}\right)_{j, l, k-1} \equiv\left(x^{\beta}\right)_{j, l, k-1}-\left(x^{\beta}\right)_{j-1, l, k-1} \\
& \Delta_{l}\left(x^{\gamma}\right)_{j-1, l, k-1} \equiv\left(x^{\gamma}\right)_{j-1, l, k-1}-\left(x^{\gamma}\right)_{j-1, l-1, k-1}
\end{aligned}
$$

for $\alpha, \beta, \gamma=0,1,2,3$ and $l=1,2, \ldots, j$ and $j=1,2, \ldots, k$ and $k=1,2, \ldots, n$.

The second combined finite hyper-surface element vector $\left(\Delta^{3} V^{\alpha \beta \gamma}\right)_{j, l, k}$ for $\alpha, \beta, \gamma=$ $0,1,2,3$ and $l=1,2, \ldots, j$ and $j=1,2, \ldots, k$ and $k=1,2, \ldots, n$ is introduced as

$$
\begin{aligned}
\left(\Delta^{3} V^{\alpha \beta \gamma}\right)_{j, l, k}= & -\Delta_{j}\left(x^{\alpha}\right)_{j, l, k} \Delta_{l}\left(x^{\beta}\right)_{j-1, l, k} \Delta_{k}\left(x^{\gamma}\right)_{j-1, l-1, k} \\
& -\Delta_{l}\left(x^{\alpha}\right)_{j, l, k} \Delta_{k}\left(x^{\beta}\right)_{j, l-1, k} \Delta_{j}\left(x^{\gamma}\right)_{j, l-1, k-1} \\
& -\Delta_{k}\left(x^{\alpha}\right)_{j, l, k} \Delta_{j}\left(x^{\beta}\right)_{j, l, k-1} \Delta_{l}\left(x^{\gamma}\right)_{j-1, l, k-1} .
\end{aligned}
$$

3. The increments of a triple sequence of points at $(l, j, k)$ are denoted as follows:

$$
\begin{aligned}
& \Delta_{l}\left(x^{\alpha}\right)_{l, j, k} \equiv\left(x^{\alpha}\right)_{l, j, k}-\left(x^{\alpha}\right)_{l-1, j, k} \\
& \Delta_{j}\left(x^{\beta}\right)_{l-1, j, k} \equiv\left(x^{\beta}\right)_{l-1, j, k}-\left(x^{\beta}\right)_{l-1, j-1, k}, \\
& \Delta_{k}\left(x^{\gamma}\right)_{l-1, j-1, k} \equiv\left(x^{\gamma}\right)_{l-1, j-1, k}-\left(x^{\gamma}\right)_{l-1, j-1, k-1} \\
& \Delta_{j}\left(x^{\alpha}\right)_{l, j, k} \equiv\left(x^{\alpha}\right)_{l, j, k}-\left(x^{\alpha}\right)_{l, j-1, k} \\
& \Delta_{k}\left(x^{\beta}\right)_{l, j-1, k} \equiv\left(x^{\beta}\right)_{l, j-1, k}-\left(x^{\beta}\right)_{l, j-1, k-1} \\
& \Delta_{l}\left(x^{\gamma}\right)_{l, j-1, k-1} \equiv\left(x^{\gamma}\right)_{l, j-1, k-1}-\left(x^{\gamma}\right)_{l-1, j-1, k-1} \\
& \Delta_{k}\left(x^{\alpha}\right)_{l, j, k} \equiv\left(x^{\alpha}\right)_{l, j, k}-\left(x^{\alpha}\right)_{l, j, k-1}
\end{aligned}
$$




$$
\begin{aligned}
& \Delta_{l}\left(x^{\beta}\right)_{l, j, k-1} \equiv\left(x^{\beta}\right)_{l, j, k-1}-\left(x^{\beta}\right)_{l-1, j, k-1}, \\
& \Delta_{j}\left(x^{\gamma}\right)_{l-1, j, k-1} \equiv\left(x^{\gamma}\right)_{l-1, j, k-1}-\left(x^{\gamma}\right)_{l-1, j-1, k-1}
\end{aligned}
$$

for $\alpha, \beta, \gamma=0,1,2,3$ and $l=1,2, \ldots, j$ and $j=1,2, \ldots, k$ and $k=1,2, \ldots, n$.

The third combined finite hyper-surface element vector $\left(\Delta^{3} V^{\alpha \beta \gamma}\right)_{l, j, k}$ for $\alpha, \beta, \gamma=$ $0,1,2,3$ and $l=1,2, \ldots, j$ and $j=1,2, \ldots, k$ and $k=1,2, \ldots, n$ is introduced as

$$
\begin{aligned}
\left(\Delta^{3} V^{\alpha \beta \gamma}\right)_{l, j, k}= & -\Delta_{l}\left(x^{\alpha}\right)_{l, j, k} \Delta_{j}\left(x^{\beta}\right)_{l-1, j, k} \Delta_{k}\left(x^{\gamma}\right)_{l-1, j-1, k} \\
& -\Delta_{j}\left(x^{\alpha}\right)_{l, j, k} \Delta_{k}\left(x^{\beta}\right)_{l, j-1, k} \Delta_{l}\left(x^{\gamma}\right)_{l, j-1, k-1} \\
& -\Delta_{k}\left(x^{\alpha}\right)_{l, j, k} \Delta_{l}\left(x^{\beta}\right)_{l, j, k-1} \Delta_{j}\left(x^{\gamma}\right)_{l-1, j, k-1} .
\end{aligned}
$$

4. The increments of a triple sequence of points at $(l, k, j)$ are denoted as follows:

$$
\begin{aligned}
& \Delta_{l}\left(x^{\alpha}\right)_{l, k, j} \equiv\left(x^{\alpha}\right)_{l, k, j}-\left(x^{\alpha}\right)_{l-1, k, j} \\
& \Delta_{k}\left(x^{\beta}\right)_{l-1, k, j} \equiv\left(x^{\beta}\right)_{l-1, k, j}-\left(x^{\beta}\right)_{l-1, k-1, j} \\
& \Delta_{j}\left(x^{\gamma}\right)_{l-1, k-1, j} \equiv\left(x^{\gamma}\right)_{l-1, k-1, j}-\left(x^{\gamma}\right)_{l-1, k-1, j-1}, \\
& \Delta_{k}\left(x^{\alpha}\right)_{l, k, j} \equiv\left(x^{\alpha}\right)_{l, k, j}-\left(x^{\alpha}\right)_{l, k-1, j} \\
& \Delta_{j}\left(x^{\beta}\right)_{l, k-1, j} \equiv\left(x^{\beta}\right)_{l, k-1, j}-\left(x^{\beta}\right)_{l, k-1, j-1}, \\
& \Delta_{l}\left(x^{\gamma}\right)_{l, k-1, j-1} \equiv\left(x^{\gamma}\right)_{l, k-1, j-1}-\left(x^{\gamma}\right)_{l-1, k-1, j-1}, \\
& \Delta_{j}\left(x^{\alpha}\right)_{l, k, j} \equiv\left(x^{\alpha}\right)_{l, k, j}-\left(x^{\alpha}\right)_{l, k, j-1}, \\
& \Delta_{l}\left(x^{\beta}\right)_{l, k, j-1} \equiv\left(x^{\beta}\right)_{l, k, j-1}-\left(x^{\beta}\right)_{l-1, k, j-1} \\
& \Delta_{k}\left(x^{\gamma}\right)_{l-1, k, j-1} \equiv\left(x^{\gamma}\right)_{l-1, k, j-1}-\left(x^{\gamma}\right)_{l-1, k-1, j-1}
\end{aligned}
$$

for $\alpha, \beta, \gamma=0,1,2,3$ and $l=1,2, \ldots, j$ and $j=1,2, \ldots, k$ and $k=1,2, \ldots, n$.

The fourth combined finite hyper-surface element vector $\left(\Delta^{3} V^{\alpha \beta \gamma}\right)_{l, k, j}$ for $\alpha, \beta, \gamma=$ $0,1,2,3$ and $l=1,2, \ldots, j$ and $j=1,2, \ldots, k$ and $k=1,2, \ldots, n$ is introduced as

$$
\begin{aligned}
\left(\Delta^{3} V^{\alpha \beta \gamma}\right)_{l, k, j}= & -\Delta_{l}\left(x^{\alpha}\right)_{l, k, j} \Delta_{k}\left(x^{\beta}\right)_{l-1, k, j} \Delta_{j}\left(x^{\gamma}\right)_{l-1, k-1, j} \\
& -\Delta_{k}\left(x^{\alpha}\right)_{l, k, j} \Delta_{j}\left(x^{\beta}\right)_{l, k-1, j} \Delta_{l}\left(x^{\gamma}\right)_{l, k-1, j-1} \\
& -\Delta_{j}\left(x^{\alpha}\right)_{l, k, j} \Delta_{l}\left(x^{\beta}\right)_{l, k, j-1} \Delta_{k}\left(x^{\gamma}\right)_{l-1, k, j-1} .
\end{aligned}
$$

5. The increments of a triple sequence of points at $(k, l, j)$ are denoted as follows:

$$
\begin{aligned}
& \Delta_{k}\left(x^{\alpha}\right)_{k, l, j} \equiv\left(x^{\alpha}\right)_{k, l, j}-\left(x^{\alpha}\right)_{k-1, l, j} \\
& \Delta_{l}\left(x^{\beta}\right)_{k-1, l, j} \equiv\left(x^{\beta}\right)_{k-1, l, j}-\left(x^{\beta}\right)_{k-1, l-1, j} \\
& \Delta_{j}\left(x^{\gamma}\right)_{k-1, l-1, j} \equiv\left(x^{\gamma}\right)_{k-1, l-1, j}-\left(x^{\gamma}\right)_{k-1, l-1, j-1} \\
& \Delta_{l}\left(x^{\alpha}\right)_{k, l, j} \equiv\left(x^{\alpha}\right)_{k, l, j}-\left(x^{\alpha}\right)_{k, l-1, j} \\
& \Delta_{j}\left(x^{\beta}\right)_{k, l-1, j} \equiv\left(x^{\beta}\right)_{k, l-1, j}-\left(x^{\beta}\right)_{k, l-1, j-1}
\end{aligned}
$$




$$
\begin{aligned}
& \Delta_{k}\left(x^{\gamma}\right)_{k, l-1, j-1} \equiv\left(x^{\gamma}\right)_{k, l-1, j-1}-\left(x^{\gamma}\right)_{k-1, l-1, j-1}, \\
& \Delta_{j}\left(x^{\alpha}\right)_{k, l, j} \equiv\left(x^{\alpha}\right)_{k, l, j}-\left(x^{\alpha}\right)_{k, l, j-1}, \\
& \Delta_{k}\left(x^{\beta}\right)_{k, l, j-1} \equiv\left(x^{\beta}\right)_{k, l, j-1}-\left(x^{\beta}\right)_{k-1, l, j-1}, \\
& \Delta_{l}\left(x^{\gamma}\right)_{k-1, l, j-1} \equiv\left(x^{\gamma}\right)_{k-1, l, j-1}-\left(x^{\gamma}\right)_{k-1, l-1, j-1}
\end{aligned}
$$

for $\alpha, \beta, \gamma=0,1,2,3$ and $l=1,2, \ldots, j$ and $j=1,2, \ldots, k$ and $k=1,2, \ldots, n$.

The fifth combined finite hyper-surface element vector $\left(\Delta^{3} V^{\alpha \beta \gamma}\right)_{k, l, j}$ for $\alpha, \beta, \gamma=0,1,2,3$ and $l=1,2, \ldots, j$ and $j=1,2, \ldots, k$ and $k=1,2, \ldots, n$ is introduced as

$$
\begin{aligned}
\left(\Delta^{3} V^{\alpha \beta \gamma}\right)_{k, l, j}= & -\Delta_{k}\left(x^{\alpha}\right)_{k, l, j} \Delta_{l}\left(x^{\beta}\right)_{k-1, l, j} \Delta_{j}\left(x^{\gamma}\right)_{k-1, l-1, j} \\
& -\Delta_{l}\left(x^{\alpha}\right)_{k, l, j} \Delta_{j}\left(x^{\beta}\right)_{k, l-1, j} \Delta_{k}\left(x^{\gamma}\right)_{k, l-1, j-1} \\
& -\Delta_{j}\left(x^{\alpha}\right)_{k, l, j} \Delta_{k}\left(x^{\beta}\right)_{k, l, j-1} \Delta_{l}\left(x^{\gamma}\right)_{k-1, l, j-1} .
\end{aligned}
$$

6 . The increments of a triple sequence of points at $(k, j, l)$ are denoted as follows:

$$
\begin{aligned}
& \Delta_{k}\left(x^{\alpha}\right)_{k, j, l} \equiv\left(x^{\alpha}\right)_{k, j, l}-\left(x^{\alpha}\right)_{k-1, j, l}, \\
& \Delta_{j}\left(x^{\beta}\right)_{k-1, j, l} \equiv\left(x^{\beta}\right)_{k-1, j, l}-\left(x^{\beta}\right)_{k-1, j-1, l}, \\
& \Delta_{l}\left(x^{\gamma}\right)_{k-1, j-1, l} \equiv\left(x^{\gamma}\right)_{k-1, j-1, l}-\left(x^{\gamma}\right)_{k-1, j-1, l-1}, \\
& \Delta_{j}\left(x^{\alpha}\right)_{k, j, l} \equiv\left(x^{\alpha}\right)_{k, j, l}-\left(x^{\alpha}\right)_{k, j-1, l,} \\
& \Delta_{l}\left(x^{\beta}\right)_{k, j-1, l} \equiv\left(x^{\beta}\right)_{k, j-1, l}-\left(x^{\beta}\right)_{k, j-1, l-1}, \\
& \Delta_{k}\left(x^{\gamma}\right)_{k, j-1, l-1} \equiv\left(x^{\gamma}\right)_{k, j-1, l-1}-\left(x^{\gamma}\right)_{k-1, j-1, l-1}, \\
& \Delta_{l}\left(x^{\alpha}\right)_{k, j, l} \equiv\left(x^{\alpha}\right)_{k, j, l}-\left(x^{\alpha}\right)_{k, j, l-1}, \\
& \Delta_{k}\left(x^{\beta}\right)_{k, j, l-1} \equiv\left(x^{\beta}\right)_{k, j, l-1}-\left(x^{\beta}\right)_{k-1, j, l-1}, \\
& \Delta_{j}\left(x^{\gamma}\right)_{k-1, j, l-1} \equiv\left(x^{\gamma}\right)_{k-1, j, l-1}-\left(x^{\gamma}\right)_{k-1, j-1, l-1}
\end{aligned}
$$

for $\alpha, \beta, \gamma=0,1,2,3$ and $l=1,2, \ldots, j$ and $j=1,2, \ldots, k$ and $k=1,2, \ldots, n$.

The sixth combined finite hyper-surface element vector $\left(\Delta^{3} V^{\alpha \beta \gamma}\right)_{k, j, l}$ for $\alpha, \beta, \gamma=$ $0,1,2,3$ and $l=1,2, \ldots, j$ and $j=1,2, \ldots, k$ and $k=1,2, \ldots, n$ is introduced as

$$
\begin{aligned}
\left(\Delta^{3} V^{\alpha \beta \gamma}\right)_{k, j, l}= & -\Delta_{k}\left(x^{\alpha}\right)_{k, j, l} \Delta_{j}\left(x^{\beta}\right)_{k-1, j, l} \Delta_{l}\left(x^{\gamma}\right)_{k-1, j-1, l} \\
& -\Delta_{j}\left(x^{\alpha}\right)_{k, j, l} \Delta_{l}\left(x^{\beta}\right)_{k, j-1, l} \Delta_{k}\left(x^{\gamma}\right)_{k, j-1, l-1} \\
& -\Delta_{l}\left(x^{\alpha}\right)_{k, j, l} \Delta_{k}\left(x^{\beta}\right)_{k, j, l-1} \Delta_{j}\left(x^{\gamma}\right)_{k-1, j, l-1} .
\end{aligned}
$$

The antisymmetric symbol in the $4 \mathrm{D}$ time-space is

$$
\varepsilon^{\alpha \beta \gamma \delta}=\varepsilon_{\alpha \beta \gamma \delta}= \begin{cases}+1 & \text { for even permutation of }\{0,1,2,3\} \\ -1 & \text { for odd permutation of }\{0,1,2,3\} \\ 0 & \text { otherwise. }\end{cases}
$$


Using the antisymmetric symbol in (4.61), the first antisymmetric finite hyper-surface element vector $\left(\Delta^{3} V_{\mu}\right)_{j, k, l}$, the second antisymmetric finite hyper-surface element vector $\left(\Delta^{3} V_{\mu}\right)_{j, l, k}$, the third antisymmetric finite hyper-surface element vector $\left(\Delta^{3} V_{\mu}\right)_{l, j, k}$, the fourth antisymmetric finite hyper-surface element vector $\left(\Delta^{3} V_{\mu}\right)_{l, k, j}$, the fifth antisymmetric finite hyper-surface element vector $\left(\Delta^{3} V_{\mu}\right)_{k, l, j}$ and the sixth antisymmetric finite hyper-surface element vector $\left(\Delta^{3} V_{\mu}\right)_{k, j, l}$ for $\mu=0,1,2,3$ and $l=1,2, \ldots, j$ and $j=1,2, \ldots, k$ and $k=1,2, \ldots, n$ are respectively introduced as

$$
\begin{aligned}
& \left(\Delta^{3} V_{\mu}\right)_{j, k, l}=\frac{1}{3 !} \varepsilon_{\alpha \beta \gamma \mu}\left(\Delta^{3} V^{\alpha \beta \gamma}\right)_{j, k, l}, \\
& \left(\Delta^{3} V_{\mu}\right)_{j, l, k}=\frac{1}{3 !} \varepsilon_{\alpha \beta \gamma \mu}\left(\Delta^{3} V^{\alpha \beta \gamma}\right)_{j, l, k}, \\
& \left(\Delta^{3} V_{\mu}\right)_{l, j, k}=\frac{1}{3 !} \varepsilon_{\alpha \beta \gamma \mu}\left(\Delta^{3} V^{\alpha \beta \gamma}\right)_{l, j, k}, \\
& \left(\Delta^{3} V_{\mu}\right)_{l, k, j}=\frac{1}{3 !} \varepsilon_{\alpha \beta \gamma \mu}\left(\Delta^{3} V^{\alpha \beta \gamma}\right)_{l, k, j}, \\
& \left(\Delta^{3} V_{\mu}\right)_{k, l, j}=\frac{1}{3 !} \varepsilon_{\alpha \beta \gamma \mu}\left(\Delta^{3} V^{\alpha \beta \gamma}\right)_{k, l, j}, \\
& \left(\Delta^{3} V_{\mu}\right)_{k, j, l}=\frac{1}{3 !} \varepsilon_{\alpha \beta \gamma \mu}\left(\Delta^{3} V^{\alpha \beta \gamma}\right)_{k, j, l},
\end{aligned}
$$

where the indices are summed over $\alpha, \beta, \gamma=0,1,2,3$.

\subsection{Triangular quadruple integral for a 4-variable function}

Assume that $D$ is a domain and $\partial D$ is the boundary of the domain in the $4 \mathrm{D}$ timespace, expressed in the Cartesian coordinates $(t, x, y, z) \in \mathbb{R}^{4}$. Let $X^{0}=T=T(t, x, y, z)$, $X^{1}=X=X(t, x, y, z), X^{2}=Y=Y(t, x, y, z)$, and $X^{3}=Z=Z(t, x, y, z)$ be partially differentiable functions with respect to $x^{0}=t, x^{1}=x, x^{2}=y$, and $x^{3}=z$ in $D$. There are $3 !=6$ kinds of triple sequences,

$$
\begin{aligned}
& \left(X^{\mu}\right)_{j, k, l}=\left(T_{j, k, l}, X_{j, k, l}, Y_{j, k, l}, Z_{j, k, l}\right), \\
& \left(X^{\mu}\right)_{j, l, k}=\left(T_{j, l, k}, X_{j, l, k}, Y_{j, l, k}, Z_{j, l, k}\right), \\
& \left(X^{\mu}\right)_{l, j, k}=\left(T_{l, j, k}, X_{l, j, k}, Y_{l, j, k}, Z_{l, j, k}\right), \\
& \left(X^{\mu}\right)_{l, k, j}=\left(T_{l, k, j}, X_{l, k, j}, Y_{l, k, j}, Z_{l, k, j}\right), \\
& \left(X^{\mu}\right)_{k, l, j}=\left(T_{k, l, j}, X_{k, l, j}, Y_{k, l, j}, Z_{k, l, j}\right), \\
& \left(X^{\mu}\right)_{k, j, l}=\left(T_{k, j, l}, X_{k, j, l}, Y_{k, j, l}, Z_{k, j, l}\right)
\end{aligned}
$$

for $\mu=0,1,2,3$ and $l=0,1,2, \ldots, j$ and $j=0,1,2, \ldots, k$ and $k=0,1,2, \ldots, n$. There are six alternatives for decomposition of six kinds of triple sequences $\left(X^{\mu}\right)_{j, k, l},\left(X^{\mu}\right)_{j, l, k},\left(X^{\mu}\right)_{l, j, k}$, $\left(X^{\mu}\right)_{l, k, j},\left(X^{\mu}\right)_{k, l, j}$, and $\left(X^{\mu}\right)_{k, j, l}$ for $\mu=0,1,2,3$ and $l=1,2, \ldots, j$ and $j=1,2, \ldots, k$ and $k=$ $1,2, \ldots, n$.

As mentioned in the Introduction, the inappropriate kind of six sets of increments, used in the previous article [6], is replaced by the appropriate kind of six sets of increments $\left\{\Delta_{h}\left(x^{\delta}\right)_{0, h, 0}, \Delta_{i}\left(x^{\delta}\right)_{i, k, 0}, \Delta_{m}\left(x^{\delta}\right)_{j, k, m}\right\},\left\{\Delta_{h}\left(x^{\delta}\right)_{0,0, h}, \Delta_{i}\left(x^{\delta}\right)_{i, 0, k}, \Delta_{m}\left(x^{\delta}\right)_{j, m, k}\right\},\left\{\Delta_{h}\left(x^{\delta}\right)_{0,0, h}\right.$, 
$\left.\Delta_{i}\left(x^{\delta}\right)_{0, i, k}, \Delta_{m}\left(x^{\delta}\right)_{m, j, k}\right\}, \quad\left\{\Delta_{h}\left(x^{\delta}\right)_{0, h, 0}, \Delta_{i}\left(x^{\delta}\right)_{0, k, i}, \Delta_{m}\left(x^{\delta}\right)_{m, k, j}\right\}, \quad\left\{\Delta_{h}\left(x^{\delta}\right)_{h, 0,0}, \Delta_{i}\left(x^{\delta}\right)_{k, 0, i}\right.$, $\left.\Delta_{m}\left(x^{\delta}\right)_{k, m, j}\right\}$ and $\left\{\Delta_{h}\left(x^{\delta}\right)_{h, 0,0}, \Delta_{i}\left(x^{\delta}\right)_{k, i, 0}, \Delta_{m}\left(x^{\delta}\right)_{k, j, m}\right\}$ for $\delta=0,1,2,3$ and $m=1,2, \ldots, l$ and $i=1,2, \ldots, j$ and $j, h=1,2, \ldots, k$ and $k=1,2, \ldots, n$ to calculate appropriate numerical values.

The following formulae have been revised based on the appropriate set of increments. In order to prove Theorem $3,\left(X^{\mu}\right)_{j, k, l},\left(X^{\mu}\right)_{j, l, k},\left(X^{\mu}\right)_{l, j, k},\left(X^{\mu}\right)_{l, k, j},\left(X^{\mu}\right)_{k, l, j}$, and $\left(X^{\mu}\right)_{k, j, l}$ for $\mu=0,1,2,3$ and $l=1,2, \ldots, j$ and $j=1,2, \ldots, k$ and $k=1,2, \ldots, n$ are respectively modified in Lemmata $4,5,6,7,8$, and 9.

There are $4 !=24$ sets of possible partial increments for a 4 -variable function.

1. The total increments of $\left(X^{\mu}\right)_{0, h, 0}$ for $\mu=0,1,2,3$ and $h=1,2, \ldots, k$ are denoted

$$
\begin{aligned}
\Delta_{h}\left(X^{\mu}\right)_{0, h, 0} & \equiv\left(X^{\mu}\right)_{0, h, 0}-\left(X^{\mu}\right)_{0, h-1,0} \\
& =X^{\mu}\left(t_{0, h, 0}, x_{0, h, 0}, y_{0, h, 0}, z_{0, h, 0}\right)-X^{\mu}\left(t_{0, h-1,0}, x_{0, h-1,0}, y_{0, h-1,0}, z_{0, h-1,0}\right) .
\end{aligned}
$$

The increments of $\Delta_{h}\left(x^{\delta}\right)_{0, h, 0}$ for $\delta=0,1,2,3$ and $h=1,2, \ldots, k$ are denoted

$$
\Delta_{h}\left(x^{\delta}\right)_{0, h, 0} \equiv\left(x^{\delta}\right)_{0, h, 0}-\left(x^{\delta}\right)_{0, h-1,0} .
$$

The sets of possible partial increments of $\Delta_{h}\left(X^{\mu}\right)_{0, h, 0}$ for $\mu=0,1,2,3$ and $h=1,2, \ldots, k$ are denoted

$$
\begin{aligned}
\Delta X^{\mu}\left[\Delta_{h} t_{0, h, 0}\right]= & X^{\mu}\left(t_{0, h, 0}, x_{0, h, 0}, y_{0, h, 0}, z_{0, h, 0}\right)-X^{\mu}\left(t_{0, h-1,0}, x_{0, h, 0}, y_{0, h, 0}, z_{0, h, 0}\right), \\
\Delta X^{\mu}\left[\Delta_{h} x_{0, h, 0}\right]= & X^{\mu}\left(t_{0, h-1,0}, x_{0, h, 0}, y_{0, h, 0}, z_{0, h, 0}\right) \\
& -X^{\mu}\left(t_{0, h-1,0}, x_{0, h-1,0}, y_{0, h, 0}, z_{0, h, 0}\right), \\
\Delta X^{\mu}\left[\Delta_{h} y_{0, h, 0}\right]= & X^{\mu}\left(t_{0, h-1,0}, x_{0, h-1,0}, y_{0, h, 0}, z_{0, h, 0}\right) \\
& -X^{\mu}\left(t_{0, h-1,0}, x_{0, h-1,0}, y_{0, h-1,0}, z_{0, h, 0}\right), \\
\Delta X^{\mu}\left[\Delta_{h} z_{0, h, 0}\right]= & X^{\mu}\left(t_{0, h-1,0}, x_{0, h-1,0}, y_{0, h-1,0}, z_{0, h, 0}\right) \\
& -X^{\mu}\left(t_{0, h-1,0}, x_{0, h-1,0}, y_{0, h-1,0}, z_{0, h-1,0}\right) .
\end{aligned}
$$

2. The total increments of $\left(X^{\mu}\right)_{i, k, 0}$ for $\mu=0,1,2,3$ and $i=1,2, \ldots, j$ and $k=1,2, \ldots, n$ are denoted

$$
\begin{aligned}
\Delta_{i}\left(X^{\mu}\right)_{i, k, 0} & \equiv\left(X^{\mu}\right)_{i, k, 0}-\left(X^{\mu}\right)_{i-1, k, 0} \\
& =X^{\mu}\left(t_{i, k, 0}, x_{i, k, 0}, y_{i, k, 0}, z_{i, k, 0}\right)-X^{\mu}\left(t_{i-1, k, 0}, x_{i-1, k, 0}, y_{i-1, k, 0}, z_{i-1, k, 0}\right) .
\end{aligned}
$$

The increments of $\Delta_{i}\left(x^{\delta}\right)_{i, k, 0}$ for $\delta=0,1,2,3$ and $i=1,2, \ldots, j$ and $k=1,2, \ldots, n$ are denoted

$$
\Delta_{i}\left(x^{\delta}\right)_{i, k, 0} \equiv\left(x^{\delta}\right)_{i, k, 0}-\left(x^{\delta}\right)_{i-1, k, 0} .
$$

The sets of possible partial increments of $\Delta_{i}\left(X^{\mu}\right)_{i, k, 0}$ for $\mu=0,1,2,3$ and $i=1,2, \ldots, j$ and $k=1,2, \ldots, n$ are denoted

$$
\begin{aligned}
& \Delta X^{\mu}\left[\Delta_{i} t_{i, k, 0}\right]=X^{\mu}\left(t_{i, k, 0}, x_{i, k, 0}, y_{i, k, 0}, z_{i, k, 0}\right)-X^{\mu}\left(t_{i-1, k, 0}, x_{i, k, 0}, y_{i, k, 0}, z_{i, k, 0}\right), \\
& \Delta X^{\mu}\left[\Delta_{i} x_{i, k, 0}\right]=X^{\mu}\left(t_{i-1, k, 0}, x_{i, k, 0}, y_{i, k, 0}, z_{i, k, 0}\right)-X^{\mu}\left(t_{i-1, k, 0}, x_{i-1, k, 0}, y_{i, k, 0}, z_{i, k, 0}\right),
\end{aligned}
$$




$$
\begin{aligned}
\Delta X^{\mu}\left[\Delta_{i} y_{i, k, 0}\right]= & X^{\mu}\left(t_{i-1, k, 0}, x_{i-1, k, 0}, y_{i, k, 0}, z_{i, k, 0}\right) \\
& -X^{\mu}\left(t_{i-1, k, 0}, x_{i-1, k, 0}, y_{i-1, k, 0}, z_{i, k, 0}\right), \\
\Delta X^{\mu}\left[\Delta_{i} z_{i, k, 0}\right]= & X^{\mu}\left(t_{i-1, k, 0}, x_{i-1, k, 0}, y_{i-1, k, 0}, z_{i, k, 0}\right) \\
& -X^{\mu}\left(t_{i-1, k, 0}, x_{i-1, k, 0}, y_{i-1, k, 0}, z_{i-1, k, 0}\right) .
\end{aligned}
$$

3. The total increments of $\left(X^{\mu}\right)_{j, k, m}$ for $\mu=0,1,2,3$ and $m=1,2, \ldots, l$ and $j=1,2, \ldots, k$ and $k=1,2, \ldots, n$ are denoted

$$
\begin{aligned}
\Delta_{m}\left(X^{\mu}\right)_{j, k, m} \equiv & \left(X^{\mu}\right)_{j, k, m}-\left(X^{\mu}\right)_{j, k, m-1} \\
= & X^{\mu}\left(t_{j, k, m}, x_{j, k, m}, y_{j, k, m}, z_{j, k, m}\right) \\
& -X^{\mu}\left(t_{j, k, m-1}, x_{j, k, m-1}, y_{j, k, m-1}, z_{j, k, m-1}\right) .
\end{aligned}
$$

The increments of $\left(x^{\delta}\right)_{j, k, m}$ for $\delta=0,1,2,3$ and $m=1,2, \ldots, l$ and $j=1,2, \ldots, k$ and $k=$ $1,2, \ldots, n$ are denoted

$$
\Delta_{m}\left(x^{\delta}\right)_{j, k, m} \equiv\left(x^{\delta}\right)_{j, k, m}-\left(x^{\delta}\right)_{j, k, m-1} .
$$

The sets of possible partial increments of $\left(X^{\mu}\right)_{j, k, m}$ for $\mu=0,1,2,3$ and $m=1,2, \ldots, l$ and $j=1,2, \ldots, k$ and $k=1,2, \ldots, n$ are denoted

$$
\begin{aligned}
\Delta X^{\mu}\left[\Delta_{m} t_{j, k, m}\right]= & X^{\mu}\left(t_{j, k, m}, x_{j, k, m}, y_{j, k, m}, z_{j, k, m}\right)-X^{\mu}\left(t_{j, k, m-1}, x_{j, k, m}, y_{j, k, m}, z_{j, k, m}\right), \\
\Delta X^{\mu}\left[\Delta_{m} x_{j, k, m}\right]= & X^{\mu}\left(t_{j, k, m-1}, x_{j, k, m}, y_{j, k, m}, z_{j, k, m}\right) \\
& -X^{\mu}\left(t_{j, k, m-1}, x_{j, k, m-1}, y_{j, k, m}, z_{j, k, m}\right), \\
\Delta X^{\mu}\left[\Delta_{m} y_{j, k, m}\right]= & X^{\mu}\left(t_{j, k, m-1}, x_{j, k, m-1}, y_{j, k, m}, z_{j, k, m}\right) \\
& -X^{\mu}\left(t_{j, k, m-1}, x_{j, k, m-1}, y_{j, k, m-1}, z_{j, k, m}\right), \\
\Delta X^{\mu}\left[\Delta_{m} z_{j, k, m}\right]= & X^{\mu}\left(t_{j, k, m-1}, x_{j, k, m-1}, y_{j, k, m-1}, z_{j, k, m}\right) \\
& -X^{\mu}\left(t_{j, k, m-1}, x_{j, k, m-1}, y_{j, k, m-1}, z_{j, k, m-1}\right) .
\end{aligned}
$$

Lemma 4 In the case of $(j, k, l)$ for $\mu=0,1,2,3$ and $l=1,2, \ldots, j$ and $j=1,2, \ldots, k$ and $k=$ $1,2, \ldots, n$, the following holds:

$$
\begin{aligned}
\left(X^{\mu}\right)_{j, k, l}= & \left(X^{\mu}\right)_{0,0,0}+\sum_{h=1}^{k} \frac{\Delta X^{\mu}\left[\Delta_{h}\left(x^{\delta}\right)_{0, h, 0}\right]}{\Delta_{h}\left(x^{\delta}\right)_{0, h, 0}} \Delta_{h}\left(x^{\delta}\right)_{0, h, 0} \\
& +\sum_{i=1}^{j} \frac{\Delta X^{\mu}\left[\Delta_{i}\left(x^{\delta}\right)_{i, k, 0}\right]}{\Delta_{i}\left(x^{\delta}\right)_{i, k, 0}} \Delta_{i}\left(x^{\delta}\right)_{i, k, 0} \\
& +\sum_{m=1}^{l} \frac{\Delta X^{\mu}\left[\Delta_{m}\left(x^{\delta}\right)_{j, k, m}\right]}{\Delta_{m}\left(x^{\delta}\right)_{j, k, m}} \Delta_{m}\left(x^{\delta}\right)_{j, k, m^{\prime}}
\end{aligned}
$$

where the index is summed over $\delta=0,1,2,3$. 
Proof Using (4.74), (4.80), and (4.86), $\left(X^{\mu}\right)_{j, k, l}$ for $\mu=0,1,2,3$ and $l=1,2, \ldots, j$ and $j=$ $1,2, \ldots, k$ and $k=1,2, \ldots, n$ is split into

$$
\left(X^{\mu}\right)_{j, k, l}=\left(X^{\mu}\right)_{0,0,0}+\sum_{h=1}^{k} \Delta_{h}\left(X^{\mu}\right)_{0, h, 0}+\sum_{i=1}^{j} \Delta_{i}\left(X^{\mu}\right)_{i, k, 0}+\sum_{m=1}^{l} \Delta_{m}\left(X^{\mu}\right)_{j, k, m} .
$$

1. Substituting (4.76), (4.77), (4.78), and (4.79) into (4.74) for $\mu=0,1,2,3$ and $h=$ $1,2, \ldots, k$, we obtain

$$
\begin{aligned}
\Delta_{h}\left(X^{\mu}\right)_{0, h, 0} & =\Delta X^{\mu}\left[\Delta_{h} t_{0, h, 0}\right]+\Delta X^{\mu}\left[\Delta_{h} x_{0, h, 0}\right]+\Delta X^{\mu}\left[\Delta_{h} y_{0, h, 0}\right]+\Delta X^{\mu}\left[\Delta_{h} z_{0, h, 0}\right] \\
& =\frac{\Delta X^{\mu}\left[\Delta_{h}\left(x^{\delta}\right)_{0, h, 0}\right]}{\Delta_{h}\left(x^{\delta}\right)_{0, h, 0}} \Delta_{h}\left(x^{\delta}\right)_{0, h, 0}
\end{aligned}
$$

where the index is summed over $\delta=0,1,2,3$.

2. Substituting (4.82), (4.83), (4.84), and (4.85) into (4.80) for $\mu=0,1,2,3$ and $i=$ $1,2, \ldots, j$ and $k=1,2, \ldots, n$, we obtain

$$
\begin{aligned}
\Delta_{i}\left(X^{\mu}\right)_{i, k, 0} & =\Delta X^{\mu}\left[\Delta_{i} t_{i, k, 0}\right]+\Delta X^{\mu}\left[\Delta_{i} x_{i, k, 0}\right]+\Delta X^{\mu}\left[\Delta_{i} y_{i, k, 0}\right]+\Delta X^{\mu}\left[\Delta_{i} z_{i, k, 0}\right] \\
& =\frac{\Delta X^{\mu}\left[\Delta_{i}\left(x^{\delta}\right)_{i, k, 0}\right]}{\Delta_{i}\left(x^{\delta}\right)_{i, k, 0}} \Delta_{i}\left(x^{\delta}\right)_{i, k, 0}
\end{aligned}
$$

where the index is summed over $\delta=0,1,2,3$.

3. Substituting (4.88), (4.89), (4.90), and (4.91) into (4.86) for $\mu=0,1,2,3$ and $m=$ $1,2, \ldots, l$ and $j=1,2, \ldots, k$ and $k=1,2, \ldots, n$, we obtain

$$
\begin{aligned}
\Delta_{m}\left(X^{\mu}\right)_{j, k, m} & =\Delta X^{\mu}\left[\Delta_{m} t_{j, k, m}\right]+\Delta X^{\mu}\left[\Delta_{m} x_{j, k, m}\right]+\Delta X^{\mu}\left[\Delta_{m} y_{j, k, m}\right]+\Delta X^{\mu}\left[\Delta_{m} z_{j, k, m}\right] \\
& =\frac{\Delta X^{\mu}\left[\Delta_{m}\left(x^{\delta}\right)_{j, k, m}\right]}{\Delta_{m}\left(x^{\delta}\right)_{j, k, m}} \Delta_{m}\left(x^{\delta}\right)_{j, k, m},
\end{aligned}
$$

where the index is summed over $\delta=0,1,2,3$.

Substituting (4.94), (4.95), and (4.96) into (4.93), we obtain (4.92).

In a similar manner, we obtain the cases of $(j, l, k),(l, j, k),(l, k, j),(k, l, j)$, and $(k, j, l)$ as follows.

Lemma 5 In the case of $(j, l, k)$ for $\mu=0,1,2,3$ and $l=1,2, \ldots, j$ and $j=1,2, \ldots, k$ and $k=$ $1,2, \ldots, n$, the following holds:

$$
\begin{aligned}
\left(X^{\mu}\right)_{j, l, k}= & \left(X^{\mu}\right)_{0,0,0}+\sum_{h=1}^{k} \frac{\Delta X^{\mu}\left[\Delta_{h}\left(x^{\delta}\right)_{0,0, h}\right]}{\Delta_{h}\left(x^{\delta}\right)_{0,0, h}} \Delta_{h}\left(x^{\delta}\right)_{0,0, h} \\
& +\sum_{i=1}^{j} \frac{\Delta X^{\mu}\left[\Delta_{i}\left(x^{\delta}\right)_{i, 0, k}\right]}{\Delta_{i}\left(x^{\delta}\right)_{i, 0, k}} \Delta_{i}\left(x^{\delta}\right)_{i, 0, k} \\
& +\sum_{m=1}^{l} \frac{\Delta X^{\mu}\left[\Delta_{m}\left(x^{\delta}\right)_{j, m, k}\right]}{\Delta_{m}\left(x^{\delta}\right)_{j, m, k}} \Delta_{m}\left(x^{\delta}\right)_{j, m, k^{\prime}}
\end{aligned}
$$

where the index is summed over $\delta=0,1,2,3$. 
Lemma 6 In the case of $(l, j, k)$ for $\mu=0,1,2,3$ and $l=1,2, \ldots, j$ and $j=1,2, \ldots, k$ and $k=$ $1,2, \ldots, n$, the following holds:

$$
\begin{aligned}
\left(X^{\mu}\right)_{l, j, k}= & \left(X^{\mu}\right)_{0,0,0}+\sum_{h=1}^{k} \frac{\Delta X^{\mu}\left[\Delta_{h}\left(x^{\delta}\right)_{0,0, h}\right]}{\Delta_{h}\left(x^{\delta}\right)_{0,0, h}} \Delta_{h}\left(x^{\delta}\right)_{0,0, h} \\
& +\sum_{i=1}^{j} \frac{\Delta X^{\mu}\left[\Delta_{i}\left(x^{\delta}\right)_{0, i, k}\right]}{\Delta_{i}\left(x^{\delta}\right)_{0, i, k}} \Delta_{i}\left(x^{\delta}\right)_{0, i, k} \\
& +\sum_{m=1}^{l} \frac{\Delta X^{\mu}\left[\Delta_{m}\left(x^{\delta}\right)_{m, j, k}\right]}{\Delta_{m}\left(x^{\delta}\right)_{m, j, k}} \Delta_{m}\left(x^{\delta}\right)_{m, j, k},
\end{aligned}
$$

where the index is summed over $\delta=0,1,2,3$.

Lemma 7 In the case of $(l, k, j)$ for $\mu=0,1,2,3$ and $l=1,2, \ldots, j$ and $j=1,2, \ldots, k$ and $k=$ $1,2, \ldots, n$, the following holds:

$$
\begin{aligned}
\left(X^{\mu}\right)_{l, k, j}= & \left(X^{\mu}\right)_{0,0,0}+\sum_{h=1}^{k} \frac{\Delta X^{\mu}\left[\Delta_{h}\left(x^{\delta}\right)_{0, h, 0}\right]}{\Delta_{h}\left(x^{\delta}\right)_{0, h, 0}} \Delta_{h}\left(x^{\delta}\right)_{0, h, 0} \\
& +\sum_{i=1}^{j} \frac{\Delta X^{\mu}\left[\Delta_{i}\left(x^{\delta}\right)_{0, k, i}\right]}{\Delta_{i}\left(x^{\delta}\right)_{0, k, i}} \Delta_{i}\left(x^{\delta}\right)_{0, k, i} \\
& +\sum_{m=1}^{l} \frac{\Delta X^{\mu}\left[\Delta_{m}\left(x^{\delta}\right)_{m, k, j}\right]}{\Delta_{m}\left(x^{\delta}\right)_{m, k, j}} \Delta_{m}\left(x^{\delta}\right)_{m, k, j}
\end{aligned}
$$

where the index is summed over $\delta=0,1,2,3$.

Lemma 8 In the case of $(k, l, j)$ for $\mu=0,1,2,3$ and $l=1,2, \ldots, j$ and $j=1,2, \ldots, k$ and $k=$ $1,2, \ldots, n$, the following holds:

$$
\begin{aligned}
\left(X^{\mu}\right)_{k, l, j}= & \left(X^{\mu}\right)_{0,0,0}+\sum_{h=1}^{k} \frac{\Delta X^{\mu}\left[\Delta_{h}\left(x^{\delta}\right)_{h, 0,0}\right]}{\Delta_{h}\left(x^{\delta}\right)_{h, 0,0}} \Delta_{h}\left(x^{\delta}\right)_{h, 0,0} \\
& +\sum_{i=1}^{j} \frac{\Delta X^{\mu}\left[\Delta_{i}\left(x^{\delta}\right)_{k, 0, i}\right]}{\Delta_{i}\left(x^{\delta}\right)_{k, 0, i}} \Delta_{i}\left(x^{\delta}\right)_{k, 0, i} \\
& +\sum_{m=1}^{l} \frac{\Delta X^{\mu}\left[\Delta_{m}\left(x^{\delta}\right)_{k, m, j}\right]}{\Delta_{m}\left(x^{\delta}\right)_{k, m, j}} \Delta_{m}\left(x^{\delta}\right)_{k, m, j}
\end{aligned}
$$

where the index is summed over $\delta=0,1,2,3$.

Lemma 9 In the case of $(k, j, l)$ for $\mu=0,1,2,3$ and $l=1,2, \ldots, j$ and $j=1,2, \ldots, k$ and $k=$ $1,2, \ldots, n$, the following holds:

$$
\left(X^{\mu}\right)_{k, j, l}=\left(X^{\mu}\right)_{0,0,0}+\sum_{h=1}^{k} \frac{\Delta X^{\mu}\left[\Delta_{h}\left(x^{\delta}\right)_{h, 0,0}\right]}{\Delta_{h}\left(x^{\delta}\right)_{h, 0,0}} \Delta_{h}\left(x^{\delta}\right)_{h, 0,0}
$$




$$
\begin{aligned}
& +\sum_{i=1}^{j} \frac{\Delta X^{\mu}\left[\Delta_{i}\left(x^{\delta}\right)_{k, i, 0}\right]}{\Delta_{i}\left(x^{\delta}\right)_{k, i, 0}} \Delta_{i}\left(x^{\delta}\right)_{k, i, 0} \\
& +\sum_{m=1}^{l} \frac{\Delta X^{\mu}\left[\Delta_{m}\left(x^{\delta}\right)_{k, j, m}\right]}{\Delta_{m}\left(x^{\delta}\right)_{k, j, m}} \Delta_{m}\left(x^{\delta}\right)_{k, j, m},
\end{aligned}
$$

where the index is summed over $\delta=0,1,2,3$.

Our triangular triple and quadruple integrals and the divergence theorem in the 4D time-space are shown as follows.

Definition 7 The triangular hyper-surface triple integral in the 4D time-space $\iiint_{\partial D} X^{\mu} d^{3} V_{\mu}$ is defined as

$$
\begin{aligned}
& \iiint_{\partial D} X^{\mu} d^{3} V_{\mu} \\
& =\lim _{n \rightarrow \infty} \sum_{k=1}^{n} \sum_{j=1}^{k} \sum_{l=1}^{j}\left[\left(X^{\mu}\right)_{j, k, l}\left(\Delta^{3} V_{\mu}\right)_{j, k, l}+\left(X^{\mu}\right)_{j, l, k}\left(\Delta^{3} V_{\mu}\right)_{j, l, k}+\left(X^{\mu}\right)_{l, j, k}\left(\Delta^{3} V_{\mu}\right)_{l, j, k}\right. \\
& \left.\quad+\left(X^{\mu}\right)_{l, k, j}\left(\Delta^{3} V_{\mu}\right)_{l, k, j}+\left(X^{\mu}\right)_{k, l, j}\left(\Delta^{3} V_{\mu}\right)_{k, l, j}+\left(X^{\mu}\right)_{k, j, l}\left(\Delta^{3} V_{\mu}\right)_{k, j, l}\right]
\end{aligned}
$$

where $\partial D$ is the boundary of a domain, $d^{3} V_{\mu}$ is the antisymmetric infinitesimal hypersurface element vector and the index is summed over $\mu=0,1,2,3$.

Definition 8 The triangular quadruple integral for integrands of which are partial differentials in the $4 \mathrm{D}$ time-space $\iiint \int_{D} \frac{\partial X^{\prime \prime \prime \mu}}{\partial x^{\prime \prime \prime}} d x^{\prime \prime \prime \delta} d^{3} V_{\mu}$ is defined as

$$
\begin{aligned}
& \iiint \int_{D} \frac{\partial X^{\prime \prime \prime} \mu}{\partial x^{\prime \prime \prime} \delta} d x^{\prime \prime \prime \delta} d^{3} V_{\mu} \\
& =\lim _{n \rightarrow \infty} \sum_{k=1}^{n} \sum_{j=1}^{k} \sum_{l=1}^{j}\left\{\left[\sum_{h=1}^{k} \frac{\Delta X^{\mu}\left[\Delta_{h}\left(x^{\delta}\right)_{0, h, 0}\right]}{\Delta_{h}\left(x^{\delta}\right)_{0, h, 0}} \Delta_{h}\left(x^{\delta}\right)_{0, h, 0}\right.\right. \\
& +\sum_{i=1}^{j} \frac{\Delta X^{\mu}\left[\Delta_{i}\left(x^{\delta}\right)_{i, k, 0}\right]}{\Delta_{i}\left(x^{\delta}\right)_{i, k, 0}} \Delta_{i}\left(x^{\delta}\right)_{i, k, 0} \\
& \left.+\sum_{m=1}^{l} \frac{\Delta X^{\mu}\left[\Delta_{m}\left(x^{\delta}\right)_{j, k, m}\right]}{\Delta_{m}\left(x^{\delta}\right)_{j, k, m}} \Delta_{m}\left(x^{\delta}\right)_{j, k, m}\right]\left(\Delta^{3} V_{\mu}\right)_{j, k, l} \\
& +\left[\sum_{h=1}^{k} \frac{\Delta X^{\mu}\left[\Delta_{h}\left(x^{\delta}\right)_{0,0, h}\right]}{\Delta_{h}\left(x^{\delta}\right)_{0,0, h}} \Delta_{h}\left(x^{\delta}\right)_{0,0, h}+\sum_{i=1}^{j} \frac{\Delta X^{\mu}\left[\Delta_{i}\left(x^{\delta}\right)_{i, 0, k}\right]}{\Delta_{i}\left(x^{\delta}\right)_{i, 0, k}} \Delta_{i}\left(x^{\delta}\right)_{i, 0, k}\right. \\
& \left.+\sum_{m=1}^{l} \frac{\Delta X^{\mu}\left[\Delta_{m}\left(x^{\delta}\right)_{j, m, k}\right]}{\Delta_{m}\left(x^{\delta}\right)_{j, m, k}} \Delta_{m}\left(x^{\delta}\right)_{j, m, k}\right]\left(\Delta^{3} V_{\mu}\right)_{j, l, k} \\
& +\left[\sum_{h=1}^{k} \frac{\Delta X^{\mu}\left[\Delta_{h}\left(x^{\delta}\right)_{0,0, h}\right]}{\Delta_{h}\left(x^{\delta}\right)_{0,0, h}} \Delta_{h}\left(x^{\delta}\right)_{0,0, h}+\sum_{i=1}^{j} \frac{\Delta X^{\mu}\left[\Delta_{i}\left(x^{\delta}\right)_{0, i, k}\right]}{\Delta_{i}\left(x^{\delta}\right)_{0, i, k}} \Delta_{i}\left(x^{\delta}\right)_{0, i, k}\right. \\
& \left.+\sum_{m=1}^{l} \frac{\Delta X^{\mu}\left[\Delta_{m}\left(x^{\delta}\right)_{m, j, k}\right]}{\Delta_{m}\left(x^{\delta}\right)_{m, j, k}} \Delta_{m}\left(x^{\delta}\right)_{m, j, k}\right]\left(\Delta^{3} V_{\mu}\right)_{l, j, k}
\end{aligned}
$$




$$
\begin{aligned}
& +\left[\sum_{h=1}^{k} \frac{\Delta X^{\mu}\left[\Delta_{h}\left(x^{\delta}\right)_{0, h, 0}\right]}{\Delta_{h}\left(x^{\delta}\right)_{0, h, 0}} \Delta_{h}\left(x^{\delta}\right)_{0, h, 0}+\sum_{i=1}^{j} \frac{\Delta X^{\mu}\left[\Delta_{i}\left(x^{\delta}\right)_{0, k, i}\right]}{\Delta_{i}\left(x^{\delta}\right)_{0, k, i}} \Delta_{i}\left(x^{\delta}\right)_{0, k, i}\right. \\
& \left.+\sum_{m=1}^{l} \frac{\Delta X^{\mu}\left[\Delta_{m}\left(x^{\delta}\right)_{m, k, j}\right]}{\Delta_{m}\left(x^{\delta}\right)_{m, k, j}} \Delta_{m}\left(x^{\delta}\right)_{m, k, j}\right]\left(\Delta^{3} V_{\mu}\right)_{l, k, j} \\
& +\left[\sum_{h=1}^{k} \frac{\Delta X^{\mu}\left[\Delta_{h}\left(x^{\delta}\right)_{h, 0,0}\right]}{\Delta_{h}\left(x^{\delta}\right)_{h, 0,0}} \Delta_{h}\left(x^{\delta}\right)_{h, 0,0}+\sum_{i=1}^{j} \frac{\Delta X^{\mu}\left[\Delta_{i}\left(x^{\delta}\right)_{k, 0, i}\right]}{\Delta_{i}\left(x^{\delta}\right)_{k, 0, i}} \Delta_{i}\left(x^{\delta}\right)_{k, 0, i}\right. \\
& \left.+\sum_{m=1}^{l} \frac{\Delta X^{\mu}\left[\Delta_{m}\left(x^{\delta}\right)_{k, m, j}\right]}{\Delta_{m}\left(x^{\delta}\right)_{k, m, j}} \Delta_{m}\left(x^{\delta}\right)_{k, m, j}\right]\left(\Delta^{3} V_{\mu}\right)_{k, l, j} \\
& +\left[\sum_{h=1}^{k} \frac{\Delta X^{\mu}\left[\Delta_{h}\left(x^{\delta}\right)_{h, 0,0}\right]}{\Delta_{h}\left(x^{\delta}\right)_{h, 0,0}} \Delta_{h}\left(x^{\delta}\right)_{h, 0,0}+\sum_{i=1}^{j} \frac{\Delta X^{\mu}\left[\Delta_{i}\left(x^{\delta}\right)_{k, i, 0}\right]}{\Delta_{i}\left(x^{\delta}\right)_{k, i, 0}} \Delta_{i}\left(x^{\delta}\right)_{k, i, 0}\right. \\
& \left.\left.+\sum_{m=1}^{l} \frac{\Delta X^{\mu}\left[\Delta_{m}\left(x^{\delta}\right)_{k, j, m}\right]}{\Delta_{m}\left(x^{\delta}\right)_{k, j, m}} \Delta_{m}\left(x^{\delta}\right)_{k, j, m}\right]\left(\Delta^{3} V_{\mu}\right)_{k, j, l}\right\}
\end{aligned}
$$

where $D$ is a domain and the indices are summed over $\delta, \mu=0,1,2,3$.

The following proposition is necessary for the condition (4.105) in Theorem 3.

Proposition 3 Denote constants as $C^{0}=C^{t}, C^{1}=C^{x}, C^{2}=C^{y}$, and $C^{3}=C^{z}$, then

$$
\begin{aligned}
C^{\mu} \iiint_{\partial D} d^{3} V_{\mu}= & C^{\mu} \lim _{n \rightarrow \infty} \sum_{k=1}^{n} \sum_{j=1}^{k} \sum_{l=1}^{j}\left[\left(\Delta^{3} V_{\mu}\right)_{j, k, l}+\left(\Delta^{3} V_{\mu}\right)_{j, l, k}+\left(\Delta^{3} V_{\mu}\right)_{l, j, k}\right. \\
& \left.+\left(\Delta^{3} V_{\mu}\right)_{l, k, j}+\left(\Delta^{3} V_{\mu}\right)_{k, l, j}+\left(\Delta^{3} V_{\mu}\right)_{k, j, l}\right]
\end{aligned}
$$

holds, where the index is summed over $\mu=0,1,2,3$.

Proof The proof of this proposition is shown in The divergence theorem of a triangular integral [6].

The following is the revised version of the theorem shown in The divergence theorem of a triangular integral [6].

Theorem 3 (The divergence theorem of the triangular integral in the 4D time-space) Assume that $D$ is a domain and $\partial D$ is the boundary of the domain in the $4 D$ time-space, expressed in the Cartesian coordinates $(t, x, y, z) \in \mathbb{R}^{4}$. Let $X^{\mu}$ for $\mu=0,1,2,3$ be a set of partially differentiable functions with respect to $x^{\nu}$ for $v=0,1,2,3$ in $D$, where $X^{\mu}=X^{\mu}\left(x^{\nu}\right)$. In the case of a closed $3 D$ hyper-surface which satisfies

$$
C^{\mu} \iiint_{\partial D} d^{3} V_{\mu}=0
$$

the divergence theorem of the triangular integral in the $4 D$ time-space holds:

$$
\oiiint_{\partial D} X^{\mu} d^{3} V_{\mu}=\iiint \int_{D} \frac{\partial X^{\prime \prime \prime \mu}}{\partial x^{\prime \prime \prime}} d x^{\prime \prime \prime \delta} d^{3} V_{\mu}
$$

where the indices are summed over $\delta, \mu=0,1,2,3$ and $C^{\mu}$ are constants. 
Proof Combining (4.92) with (4.62), combining (4.97) with (4.63), combining (4.98) with (4.64), combining (4.99) with (4.65), combining (4.100) with (4.66) and combining (4.101) with (4.67) for $l=1,2, \ldots, j$ and $j=1,2, \ldots, k$ and $k=1,2, \ldots, n$, we obtain

$$
\begin{aligned}
& \sum_{k=1}^{n} \sum_{j=1}^{k} \sum_{l=1}^{j}\left[\left(X^{\mu}\right)_{j, k, l}\left(\Delta^{3} V_{\mu}\right)_{j, k, l}+\left(X^{\mu}\right)_{j, l, k}\left(\Delta^{3} V_{\mu}\right)_{j, l, k}+\left(X^{\mu}\right)_{l, j, k}\left(\Delta^{3} V_{\mu}\right)_{l, j, k}\right. \\
& \left.+\left(X^{\mu}\right)_{l, k, j}\left(\Delta^{3} V_{\mu}\right)_{l, k, j}+\left(X^{\mu}\right)_{k, l, j}\left(\Delta^{3} V_{\mu}\right)_{k, l, j}+\left(X^{\mu}\right)_{k, j, l}\left(\Delta^{3} V_{\mu}\right)_{k, j, l}\right] \\
& =\sum_{k=1}^{n} \sum_{j=1}^{k} \sum_{l=1}^{j}\left\{\left[\sum_{h=1}^{k} \frac{\Delta X^{\mu}\left[\Delta_{h}\left(x^{\delta}\right)_{0, h, 0}\right]}{\Delta_{h}\left(x^{\delta}\right)_{0, h, 0}} \Delta_{h}\left(x^{\delta}\right)_{0, h, 0}+\sum_{i=1}^{j} \frac{\Delta X^{\mu}\left[\Delta_{i}\left(x^{\delta}\right)_{i, k, 0}\right]}{\Delta_{i}\left(x^{\delta}\right)_{i, k, 0}} \Delta_{i}\left(x^{\delta}\right)_{i, k, 0}\right.\right. \\
& \left.+\sum_{m=1}^{l} \frac{\Delta X^{\mu}\left[\Delta_{m}\left(x^{\delta}\right)_{j, k, m}\right]}{\Delta_{m}\left(x^{\delta}\right)_{j, k, m}} \Delta_{m}\left(x^{\delta}\right)_{j, k, m}\right]\left(\Delta^{3} V_{\mu}\right)_{j, k, l} \\
& +\left[\sum_{h=1}^{k} \frac{\Delta X^{\mu}\left[\Delta_{h}\left(x^{\delta}\right)_{0,0, h}\right]}{\Delta_{h}\left(x^{\delta}\right)_{0,0, h}} \Delta_{h}\left(x^{\delta}\right)_{0,0, h}+\sum_{i=1}^{j} \frac{\Delta X^{\mu}\left[\Delta_{i}\left(x^{\delta}\right)_{i, 0, k}\right]}{\Delta_{i}\left(x^{\delta}\right)_{i, 0, k}} \Delta_{i}\left(x^{\delta}\right)_{i, 0, k}\right. \\
& \left.+\sum_{m=1}^{l} \frac{\Delta X^{\mu}\left[\Delta_{m}\left(x^{\delta}\right)_{j, m, k}\right]}{\Delta_{m}\left(x^{\delta}\right)_{j, m, k}} \Delta_{m}\left(x^{\delta}\right)_{j, m, k}\right]\left(\Delta^{3} V_{\mu}\right)_{j, l, k} \\
& +\left[\sum_{h=1}^{k} \frac{\Delta X^{\mu}\left[\Delta_{h}\left(x^{\delta}\right)_{0,0, h}\right]}{\Delta_{h}\left(x^{\delta}\right)_{0,0, h}} \Delta_{h}\left(x^{\delta}\right)_{0,0, h}+\sum_{i=1}^{j} \frac{\Delta X^{\mu}\left[\Delta_{i}\left(x^{\delta}\right)_{0, i, k}\right]}{\Delta_{i}\left(x^{\delta}\right)_{0, i, k}} \Delta_{i}\left(x^{\delta}\right)_{0, i, k}\right. \\
& \left.+\sum_{m=1}^{l} \frac{\Delta X^{\mu}\left[\Delta_{m}\left(x^{\delta}\right)_{m, j, k}\right]}{\Delta_{m}\left(x^{\delta}\right)_{m, j, k}} \Delta_{m}\left(x^{\delta}\right)_{m, j, k}\right]\left(\Delta^{3} V_{\mu}\right)_{l, j, k} \\
& +\left[\sum_{h=1}^{k} \frac{\Delta X^{\mu}\left[\Delta_{h}\left(x^{\delta}\right)_{0, h, 0}\right]}{\Delta_{h}\left(x^{\delta}\right)_{0, h, 0}} \Delta_{h}\left(x^{\delta}\right)_{0, h, 0}+\sum_{i=1}^{j} \frac{\Delta X^{\mu}\left[\Delta_{i}\left(x^{\delta}\right)_{0, k, i}\right]}{\Delta_{i}\left(x^{\delta}\right)_{0, k, i}} \Delta_{i}\left(x^{\delta}\right)_{0, k, i}\right. \\
& \left.+\sum_{m=1}^{l} \frac{\Delta X^{\mu}\left[\Delta_{m}\left(x^{\delta}\right)_{m, k, j}\right]}{\Delta_{m}\left(x^{\delta}\right)_{m, k, j}} \Delta_{m}\left(x^{\delta}\right)_{m, k, j}\right]\left(\Delta^{3} V_{\mu}\right)_{l, k, j} \\
& +\left[\sum_{h=1}^{k} \frac{\Delta X^{\mu}\left[\Delta_{h}\left(x^{\delta}\right)_{h, 0,0}\right]}{\Delta_{h}\left(x^{\delta}\right)_{h, 0,0}} \Delta_{h}\left(x^{\delta}\right)_{h, 0,0}+\sum_{i=1}^{j} \frac{\Delta X^{\mu}\left[\Delta_{i}\left(x^{\delta}\right)_{k, 0, i}\right]}{\Delta_{i}\left(x^{\delta}\right)_{k, 0, i}} \Delta_{i}\left(x^{\delta}\right)_{k, 0, i}\right. \\
& \left.+\sum_{m=1}^{l} \frac{\Delta X^{\mu}\left[\Delta_{m}\left(x^{\delta}\right)_{k, m, j}\right]}{\Delta_{m}\left(x^{\delta}\right)_{k, m, j}} \Delta_{m}\left(x^{\delta}\right)_{k, m, j}\right]\left(\Delta^{3} V_{\mu}\right)_{k, l, j} \\
& +\left[\sum_{h=1}^{k} \frac{\Delta X^{\mu}\left[\Delta_{h}\left(x^{\delta}\right)_{h, 0,0}\right]}{\Delta_{h}\left(x^{\delta}\right)_{h, 0,0}} \Delta_{h}\left(x^{\delta}\right)_{h, 0,0}+\sum_{i=1}^{j} \frac{\Delta X^{\mu}\left[\Delta_{i}\left(x^{\delta}\right)_{k, i, 0}\right]}{\Delta_{i}\left(x^{\delta}\right)_{k, i, 0}} \Delta_{i}\left(x^{\delta}\right)_{k, i, 0}\right. \\
& \left.\left.+\sum_{m=1}^{l} \frac{\Delta X^{\mu}\left[\Delta_{m}\left(x^{\delta}\right)_{k, j, m}\right]}{\Delta_{m}\left(x^{\delta}\right)_{k, j, m}} \Delta_{m}\left(x^{\delta}\right)_{k, j, m}\right]\left(\Delta^{3} V_{\mu}\right)_{k, j, l}\right\} \\
& +\left(X^{\mu}\right)_{0,0,0} \sum_{k=1}^{n} \sum_{j=1}^{k} \sum_{l=1}^{j}\left[\left(\Delta^{3} V_{\mu}\right)_{j, k, l}+\left(\Delta^{3} V_{\mu}\right)_{j, l, k}+\left(\Delta^{3} V_{\mu}\right)_{l, j, k}\right. \\
& \left.+\left(\Delta^{3} V_{\mu}\right)_{l, k, j}+\left(\Delta^{3} V_{\mu}\right)_{k, l, j}+\left(\Delta^{3} V_{\mu}\right)_{k, j, l}\right] \text {, }
\end{aligned}
$$


where the indices are summed over $\delta, \mu=0,1,2,3$. Using Proposition 3, (4.105) is rewritten as

$$
\begin{aligned}
& \left(X^{\mu}\right)_{0,0,0} \lim _{n \rightarrow \infty} \sum_{k=1}^{n} \sum_{j=1}^{k} \sum_{l=1}^{j}\left[\left(\Delta^{3} V_{\mu}\right)_{j, k, l}+\left(\Delta^{3} V_{\mu}\right)_{j, l, k}+\left(\Delta^{3} V_{\mu}\right)_{l, j, k}\right. \\
& \left.+\left(\Delta^{3} V_{\mu}\right)_{l, k, j}+\left(\Delta^{3} V_{\mu}\right)_{k, l, j}+\left(\Delta^{3} V_{\mu}\right)_{k, j, l}\right]=0,
\end{aligned}
$$

where the index is summed over $\mu=0,1,2,3$. The limit at infinity $n \rightarrow \infty$ of (4.107) is expressed as (4.106) by Definitions 7 and 8 under the condition of a closed hyper-surface, (4.108).

The triangular quadruple integral for a 4-variable function $w=w(t, x, y, z)$ in the 4D time-space by the infinitesimal hyper-volume element $d^{4} \Omega$ is given as

$$
\iiint \int_{D} w d^{4} \Omega=\frac{1}{4} \iiint \int_{D} w d x^{\prime \prime \prime} d^{3} V_{\delta}
$$

where the index is summed over $\delta=0,1,2,3$. In component representation, (4.109) is expressed as

$$
\begin{aligned}
\iiint \int_{D} w d^{4} \Omega= & \frac{1}{4} \iiint \int_{D} w d t^{\prime \prime \prime} d^{3} V_{t}+\frac{1}{4} \iiint \int_{D} w d x^{\prime \prime \prime} d^{3} V_{x} \\
& +\frac{1}{4} \iiint \int_{D} w d y^{\prime \prime \prime} d^{3} V_{y}+\frac{1}{4} \iiint \int_{D} w d z^{\prime \prime \prime} d^{3} V_{z}
\end{aligned}
$$

The calculation process of the triangular quadruple integral in the 4D time-space is precisely defined as follows.

Let $w=w(t, x, y, z)$ be a piecewise smooth function in the 4D time-space, expressed in the Cartesian coordinates $(t, x, y, z) \in \mathbb{R}^{4}$.

Definition 9 The triangular quadruple integral for a 4-variable function $w=w(t, x, y, z)$ in the $4 \mathrm{D}$ time-space $\frac{1}{4} \iiint \int_{D} w d x^{\prime \prime \prime} \delta d^{3} V_{\delta}$ is defined as

$$
\begin{aligned}
& \frac{1}{4} \iiint \int_{D} w d x^{\prime \prime \prime l} d^{3} V_{\delta} \\
& =\frac{1}{4} \lim _{n \rightarrow \infty} \sum_{k=1}^{n} \sum_{j=1}^{k} \sum_{l=1}^{j}\left\{\left[\sum_{h=1}^{k} w\left(\left(x^{\alpha}\right)_{j, k, l}\left(x^{\beta}\right)_{j, k, l}\left(x^{\gamma}\right)_{j, k, l}\left(x^{\delta}\right)_{0, h, 0}\right) \Delta_{h}\left(x^{\delta}\right)_{0, h, 0}\right.\right. \\
& \quad+\sum_{i=1}^{j} w\left(\left(x^{\alpha}\right)_{j, k, l}\left(x^{\beta}\right)_{j, k, l},\left(x^{\gamma}\right)_{j, k, l}\left(x^{\delta}\right)_{i, k, 0}\right) \Delta_{i}\left(x^{\delta}\right)_{i, k, 0} \\
& \left.\quad+\sum_{m=1}^{l} w\left(\left(x^{\alpha}\right)_{j, k, l}\left(x^{\beta}\right)_{j, k, l},\left(x^{\gamma}\right)_{j, k, l}\left(x^{\delta}\right)_{j, k, m}\right) \Delta_{m}\left(x^{\delta}\right)_{j, k, m}\right]\left(\Delta^{3} V_{\delta}\right)_{j, k, l} \\
& \quad+\left[\sum_{h=1}^{k} w\left(\left(x^{\alpha}\right)_{j, l, k},\left(x^{\beta}\right)_{j, l, k},\left(x^{\gamma}\right)_{j, l, k},\left(x^{\delta}\right)_{0,0, h}\right) \Delta_{h}\left(x^{\delta}\right)_{0,0, h}\right. \\
& \quad+\sum_{i=1}^{j} w\left(\left(x^{\alpha}\right)_{j, l, k},\left(x^{\beta}\right)_{j, l, k},\left(x^{\gamma}\right)_{j, l, k},\left(x^{\delta}\right)_{i, 0, k}\right) \Delta_{i}\left(x^{\delta}\right)_{i, 0, k}
\end{aligned}
$$




$$
\begin{aligned}
& \left.+\sum_{m=1}^{l} w\left(\left(x^{\alpha}\right)_{j, l, k},\left(x^{\beta}\right)_{j, l, k},\left(x^{\gamma}\right)_{j, l, k},\left(x^{\delta}\right)_{j, m, k}\right) \Delta_{m}\left(x^{\delta}\right)_{j, m, k}\right]\left(\Delta^{3} V_{\delta}\right)_{j, l, k} \\
& +\left[\sum_{h=1}^{k} w\left(\left(x^{\alpha}\right)_{l, j, k},\left(x^{\beta}\right)_{l, j, k},\left(x^{\gamma}\right)_{l, j, k},\left(x^{\delta}\right)_{0,0, h}\right) \Delta_{h}\left(x^{\delta}\right)_{0,0, h}\right. \\
& +\sum_{i=1}^{j} w\left(\left(x^{\alpha}\right)_{l, j, k},\left(x^{\beta}\right)_{l, j, k},\left(x^{\gamma}\right)_{l, j, k},\left(x^{\delta}\right)_{0, i, k}\right) \Delta_{i}\left(x^{\delta}\right)_{0, i, k} \\
& \left.+\sum_{m=1}^{l} w\left(\left(x^{\alpha}\right)_{l, j, k},\left(x^{\beta}\right)_{l, j, k},\left(x^{\gamma}\right)_{l, j, k},\left(x^{\delta}\right)_{m, j, k}\right) \Delta_{m}\left(x^{\delta}\right)_{m, j, k}\right]\left(\Delta^{3} V_{\delta}\right)_{l, j, k} \\
& +\left[\sum_{h=1}^{k} w\left(\left(x^{\alpha}\right)_{l, k, j},\left(x^{\beta}\right)_{l, k, j^{\prime}}\left(x^{\gamma}\right)_{l, k, j^{\prime}}\left(x^{\delta}\right)_{0, h, 0}\right) \Delta_{h}\left(x^{\delta}\right)_{0, h, 0}\right. \\
& +\sum_{i=1}^{j} w\left(\left(x^{\alpha}\right)_{l, k, j^{\prime}}\left(x^{\beta}\right)_{l, k, j^{\prime}}\left(x^{\gamma}\right)_{l, k, j^{\prime}}\left(x^{\delta}\right)_{0, k, i}\right) \Delta_{i}\left(x^{\delta}\right)_{0, k, i} \\
& \left.+\sum_{m=1}^{l} w\left(\left(x^{\alpha}\right)_{l, k, j^{\prime}}\left(x^{\beta}\right)_{l, k, j^{\prime}}\left(x^{\gamma}\right)_{l, k, j^{\prime}},\left(x^{\delta}\right)_{m, k, j}\right) \Delta_{m}\left(x^{\delta}\right)_{m, k, j}\right]\left(\Delta^{3} V_{\delta}\right)_{l, k, j} \\
& +\left[\sum_{h=1}^{k} w\left(\left(x^{\alpha}\right)_{k, l, j},\left(x^{\beta}\right)_{k, l, j},\left(x^{\gamma}\right)_{k, l, j},\left(x^{\delta}\right)_{h, 0,0}\right) \Delta_{h}\left(x^{\delta}\right)_{h, 0,0}\right. \\
& +\sum_{i=1}^{j} w\left(\left(x^{\alpha}\right)_{k, l, j},\left(x^{\beta}\right)_{k, l, j},\left(x^{\gamma}\right)_{k, l, j},\left(x^{\delta}\right)_{k, 0, i}\right) \Delta_{i}\left(x^{\delta}\right)_{k, 0, i} \\
& \left.+\sum_{m=1}^{l} w\left(\left(x^{\alpha}\right)_{k, l, j^{\prime}}\left(x^{\beta}\right)_{k, l, j},\left(x^{\gamma}\right)_{k, l, j^{\prime}}\left(x^{\delta}\right)_{k, m, j}\right) \Delta_{m}\left(x^{\delta}\right)_{k, m, j}\right]\left(\Delta^{3} V_{\delta}\right)_{k, l, j} \\
& +\left[\sum_{h=1}^{k} w\left(\left(x^{\alpha}\right)_{k, j, l},\left(x^{\beta}\right)_{k, j, l},\left(x^{\gamma}\right)_{k, j, l},\left(x^{\delta}\right)_{h, 0,0}\right) \Delta_{h}\left(x^{\delta}\right)_{h, 0,0}\right. \\
& +\sum_{i=1}^{j} w\left(\left(x^{\alpha}\right)_{k, j, l},\left(x^{\beta}\right)_{k, j, l},\left(x^{\gamma}\right)_{k, j, l},\left(x^{\delta}\right)_{k, i, 0}\right) \Delta_{i}\left(x^{\delta}\right)_{k, i, 0} \\
& \left.\left.+\sum_{m=1}^{l} w\left(\left(x^{\alpha}\right)_{k, j, l},\left(x^{\beta}\right)_{k, j, l}\left(x^{\gamma}\right)_{k, j, l},\left(x^{\delta}\right)_{k, j, m}\right) \Delta_{m}\left(x^{\delta}\right)_{k, j, m}\right]\left(\Delta^{3} V_{\delta}\right)_{k, j, l}\right\}
\end{aligned}
$$

for $\alpha \neq \beta, \alpha \neq \gamma, \alpha \neq \delta, \beta \neq \gamma, \beta \neq \delta, \gamma \neq \delta$ and $\alpha, \beta, \gamma=0,1,2,3$ as the indices of variable of function $w=w(t, x, y, z)$, where $D$ is a domain and the index is summed over $\delta=0,1,2,3$.

The revised corollary shown below derived from Theorem 3 is the $4 \mathrm{D}$ version of Corollary 2.

Corollary 3 (A corollary of Theorem 3) Assume that $D$ is a domain and $\partial D$ is the boundary of the domain in the $4 D$ time-space, expressed in the Cartesian coordinates $(t, x, y, z) \in \mathbb{R}^{4}$. Let $X^{\mu}$ for $\mu=0,1,2,3$ be a set of partially differentiable functions with respect to $x^{v}$ for $v=0,1,2,3$ in $D$, where $X^{\mu}=X^{\mu}\left(x^{v}\right)$. In the case of

$$
\oiiint_{\partial D} X^{\mu} d^{3} V_{\mu}=\iiint \int_{D} w d^{4} \Omega
$$


where $w=w(t, x, y, z)$ is a 4-variable function and the index is summed over $\mu=0,1,2,3$, the following holds:

$$
\frac{\partial X^{\mu}}{\partial x^{\mu}}=w
$$

Proof Substituting (4.106) and (4.109) into (4.112), it is rewritten as

$$
\iiint \int_{D} \frac{\partial X^{\prime \prime \prime} \mu}{\partial x^{\prime \prime \prime}} d x^{\prime \prime \prime \delta} d^{3} V_{\mu}=\frac{1}{4} \iiint \int_{D} w d x^{\prime \prime \prime \delta} d^{3} V_{\delta}
$$

where the indices are summed over $\delta, \mu=0,1,2,3$. Substituting (4.103) and (4.111) into (4.114), it is expressed as

$$
\begin{aligned}
& \lim _{n \rightarrow \infty} \sum_{k=1}^{n} \sum_{j=1}^{k} \sum_{l=1}^{j}\left\{\left[\sum_{h=1}^{k} \frac{\Delta X^{\mu}\left[\Delta_{h}\left(x^{\delta}\right)_{0, h, 0}\right]}{\Delta_{h}\left(x^{\delta}\right)_{0, h, 0}} \Delta_{h}\left(x^{\delta}\right)_{0, h, 0}\right.\right. \\
& +\sum_{i=1}^{j} \frac{\Delta X^{\mu}\left[\Delta_{i}\left(x^{\delta}\right)_{i, k, 0}\right]}{\Delta_{i}\left(x^{\delta}\right)_{i, k, 0}} \Delta_{i}\left(x^{\delta}\right)_{i, k, 0} \\
& \left.+\sum_{m=1}^{l} \frac{\Delta X^{\mu}\left[\Delta_{m}\left(x^{\delta}\right)_{j, k, m}\right]}{\Delta_{m}\left(x^{\delta}\right)_{j, k, m}} \Delta_{m}\left(x^{\delta}\right)_{j, k, m}\right] \frac{1}{3 !} \varepsilon_{\alpha \beta \gamma \mu}\left(\Delta^{3} V^{\alpha \beta \gamma}\right)_{j, k, l} \\
& +\left[\sum_{h=1}^{k} \frac{\Delta X^{\mu}\left[\Delta_{h}\left(x^{\delta}\right)_{0,0, h}\right]}{\Delta_{h}\left(x^{\delta}\right)_{0,0, h}} \Delta_{h}\left(x^{\delta}\right)_{0,0, h}+\sum_{i=1}^{j} \frac{\Delta X^{\mu}\left[\Delta_{i}\left(x^{\delta}\right)_{i, 0, k}\right]}{\Delta_{i}\left(x^{\delta}\right)_{i, 0, k}} \Delta_{i}\left(x^{\delta}\right)_{i, 0, k}\right. \\
& \left.+\sum_{m=1}^{l} \frac{\Delta X^{\mu}\left[\Delta_{m}\left(x^{\delta}\right)_{j, m, k}\right]}{\Delta_{m}\left(x^{\delta}\right)_{j, m, k}} \Delta_{m}\left(x^{\delta}\right)_{j, m, k}\right] \frac{1}{3 !} \varepsilon_{\alpha \beta \gamma \mu}\left(\Delta^{3} V^{\alpha \beta \gamma}\right)_{j, l, k} \\
& +\left[\sum_{h=1}^{k} \frac{\Delta X^{\mu}\left[\Delta_{h}\left(x^{\delta}\right)_{0,0, h}\right]}{\Delta_{h}\left(x^{\delta}\right)_{0,0, h}} \Delta_{h}\left(x^{\delta}\right)_{0,0, h}+\sum_{i=1}^{j} \frac{\Delta X^{\mu}\left[\Delta_{i}\left(x^{\delta}\right)_{0, i, k}\right]}{\Delta_{i}\left(x^{\delta}\right)_{0, i, k}} \Delta_{i}\left(x^{\delta}\right)_{0, i, k}\right. \\
& \left.+\sum_{m=1}^{l} \frac{\Delta X^{\mu}\left[\Delta_{m}\left(x^{\delta}\right)_{m, j, k}\right]}{\Delta_{m}\left(x^{\delta}\right)_{m, j, k}} \Delta_{m}\left(x^{\delta}\right)_{m, j, k}\right] \frac{1}{3 !} \varepsilon_{\alpha \beta \gamma \mu}\left(\Delta^{3} V^{\alpha \beta \gamma}\right)_{l, j, k} \\
& +\left[\sum_{h=1}^{k} \frac{\Delta X^{\mu}\left[\Delta_{h}\left(x^{\delta}\right)_{0, h, 0}\right]}{\Delta_{h}\left(x^{\delta}\right)_{0, h, 0}} \Delta_{h}\left(x^{\delta}\right)_{0, h, 0}+\sum_{i=1}^{j} \frac{\Delta X^{\mu}\left[\Delta_{i}\left(x^{\delta}\right)_{0, k, i}\right]}{\Delta_{i}\left(x^{\delta}\right)_{0, k, i}} \Delta_{i}\left(x^{\delta}\right)_{0, k, i}\right. \\
& \left.+\sum_{m=1}^{l} \frac{\Delta X^{\mu}\left[\Delta_{m}\left(x^{\delta}\right)_{m, k, j}\right]}{\Delta_{m}\left(x^{\delta}\right)_{m, k, j}} \Delta_{m}\left(x^{\delta}\right)_{m, k, j}\right] \frac{1}{3 !} \varepsilon_{\alpha \beta \gamma \mu}\left(\Delta^{3} V^{\alpha \beta \gamma}\right)_{l, k, j} \\
& +\left[\sum_{h=1}^{k} \frac{\Delta X^{\mu}\left[\Delta_{h}\left(x^{\delta}\right)_{h, 0,0}\right]}{\Delta_{h}\left(x^{\delta}\right)_{h, 0,0}} \Delta_{h}\left(x^{\delta}\right)_{h, 0,0}+\sum_{i=1}^{j} \frac{\Delta X^{\mu}\left[\Delta_{i}\left(x^{\delta}\right)_{k, 0, i}\right]}{\Delta_{i}\left(x^{\delta}\right)_{k, 0, i}} \Delta_{i}\left(x^{\delta}\right)_{k, 0, i}\right. \\
& \left.+\sum_{m=1}^{l} \frac{\Delta X^{\mu}\left[\Delta_{m}\left(x^{\delta}\right)_{k, m, j}\right]}{\Delta_{m}\left(x^{\delta}\right)_{k, m, j}} \Delta_{m}\left(x^{\delta}\right)_{k, m, j}\right] \frac{1}{3 !} \varepsilon_{\alpha \beta \gamma \mu}\left(\Delta^{3} V^{\alpha \beta \gamma}\right)_{k, l, j} \\
& +\left[\sum_{h=1}^{k} \frac{\Delta X^{\mu}\left[\Delta_{h}\left(x^{\delta}\right)_{h, 0,0}\right]}{\Delta_{h}\left(x^{\delta}\right)_{h, 0,0}} \Delta_{h}\left(x^{\delta}\right)_{h, 0,0}+\sum_{i=1}^{j} \frac{\Delta X^{\mu}\left[\Delta_{i}\left(x^{\delta}\right)_{k, i, 0}\right]}{\Delta_{i}\left(x^{\delta}\right)_{k, i, 0}} \Delta_{i}\left(x^{\delta}\right)_{k, i, 0}\right.
\end{aligned}
$$


Tokunaga Advances in Difference Equations 2014, 2014:89

Page 38 of 53

http://www.advancesindifferenceequations.com/content/2014/1/89

$$
\begin{aligned}
& \left.\left.+\sum_{m=1}^{l} \frac{\Delta X^{\mu}\left[\Delta_{m}\left(x^{\delta}\right)_{k, j, m}\right]}{\Delta_{m}\left(x^{\delta}\right)_{k, j, m}} \Delta_{m}\left(x^{\delta}\right)_{k, j, m}\right] \frac{1}{3 !} \varepsilon_{\alpha \beta \gamma \mu}\left(\Delta^{3} V^{\alpha \beta \gamma}\right)_{k, j, l}\right\} \\
& =\frac{1}{4} \lim _{n \rightarrow \infty} \sum_{k=1}^{n} \sum_{j=1}^{k} \sum_{l=1}^{j}\left\{\left[\sum_{h=1}^{k} w\left(\left(x^{\alpha}\right)_{j, k, l},\left(x^{\beta}\right)_{j, k, l},\left(x^{\gamma}\right)_{j, k, l},\left(x^{\delta}\right)_{0, h, 0}\right) \Delta_{h}\left(x^{\delta}\right)_{0, h, 0}\right.\right. \\
& +\sum_{i=1}^{j} w\left(\left(x^{\alpha}\right)_{j, k, l}\left(x^{\beta}\right)_{j, k, l},\left(x^{\gamma}\right)_{j, k, l},\left(x^{\delta}\right)_{i, k, 0}\right) \Delta_{i}\left(x^{\delta}\right)_{i, k, 0} \\
& \left.+\sum_{m=1}^{l} w\left(\left(x^{\alpha}\right)_{j, k, l}\left(x^{\beta}\right)_{j, k, l}\left(x^{\gamma}\right)_{j, k, l}\left(x^{\delta}\right)_{j, k, m}\right) \Delta_{m}\left(x^{\delta}\right)_{j, k, m}\right] \frac{1}{3 !} \varepsilon_{\alpha \beta \gamma \delta}\left(\Delta^{3} V^{\alpha \beta \gamma}\right)_{j, k, l} \\
& +\left[\sum_{h=1}^{k} w\left(\left(x^{\alpha}\right)_{j, l, k},\left(x^{\beta}\right)_{j, l, k},\left(x^{\gamma}\right)_{j, l, k},\left(x^{\delta}\right)_{0,0, h}\right) \Delta_{h}\left(x^{\delta}\right)_{0,0, h}\right. \\
& +\sum_{i=1}^{j} w\left(\left(x^{\alpha}\right)_{j, l, k},\left(x^{\beta}\right)_{j, l, k},\left(x^{\gamma}\right)_{j, l, k},\left(x^{\delta}\right)_{i, 0, k}\right) \Delta_{i}\left(x^{\delta}\right)_{i, 0, k} \\
& \left.+\sum_{m=1}^{l} w\left(\left(x^{\alpha}\right)_{j, l, k},\left(x^{\beta}\right)_{j, l, k},\left(x^{\gamma}\right)_{j, l, k},\left(x^{\delta}\right)_{j, m, k}\right) \Delta_{m}\left(x^{\delta}\right)_{j, m, k}\right] \frac{1}{3 !} \varepsilon_{\alpha \beta \gamma \delta}\left(\Delta^{3} V^{\alpha \beta \gamma}\right)_{j, l, k} \\
& +\left[\sum_{h=1}^{k} w\left(\left(x^{\alpha}\right)_{l, j, k},\left(x^{\beta}\right)_{l, j, k},\left(x^{\gamma}\right)_{l, j, k},\left(x^{\delta}\right)_{0,0, h}\right) \Delta_{h}\left(x^{\delta}\right)_{0,0, h}\right. \\
& +\sum_{i=1}^{j} w\left(\left(x^{\alpha}\right)_{l, j, k},\left(x^{\beta}\right)_{l, j, k},\left(x^{\gamma}\right)_{l, j, k},\left(x^{\delta}\right)_{0, i, k}\right) \Delta_{i}\left(x^{\delta}\right)_{0, i, k} \\
& \left.+\sum_{m=1}^{l} w\left(\left(x^{\alpha}\right)_{l, j, k},\left(x^{\beta}\right)_{l, j, k},\left(x^{\gamma}\right)_{l, j, k},\left(x^{\delta}\right)_{m, j, k}\right) \Delta_{m}\left(x^{\delta}\right)_{m, j, k}\right] \frac{1}{3 !} \varepsilon_{\alpha \beta \gamma \delta}\left(\Delta^{3} V^{\alpha \beta \gamma}\right)_{l, j, k} \\
& +\left[\sum_{h=1}^{k} w\left(\left(x^{\alpha}\right)_{l, k, j},\left(x^{\beta}\right)_{l, k, j},\left(x^{\gamma}\right)_{l, k, j},\left(x^{\delta}\right)_{0, h, 0}\right) \Delta_{h}\left(x^{\delta}\right)_{0, h, 0}\right. \\
& +\sum_{i=1}^{j} w\left(\left(x^{\alpha}\right)_{l, k, j},\left(x^{\beta}\right)_{l, k, j},\left(x^{\gamma}\right)_{l, k, j},\left(x^{\delta}\right)_{0, k, i}\right) \Delta_{i}\left(x^{\delta}\right)_{0, k, i} \\
& \left.+\sum_{m=1}^{l} w\left(\left(x^{\alpha}\right)_{l, k, j},\left(x^{\beta}\right)_{l, k, j},\left(x^{\gamma}\right)_{l, k, j},\left(x^{\delta}\right)_{m, k, j}\right) \Delta_{m}\left(x^{\delta}\right)_{m, k, j}\right] \frac{1}{3 !} \varepsilon_{\alpha \beta \gamma \delta}\left(\Delta^{3} V^{\alpha \beta \gamma}\right)_{l, k, j} \\
& +\left[\sum_{h=1}^{k} w\left(\left(x^{\alpha}\right)_{k, l, j},\left(x^{\beta}\right)_{k, l, j},\left(x^{\gamma}\right)_{k, l, j},\left(x^{\delta}\right)_{h, 0,0}\right) \Delta_{h}\left(x^{\delta}\right)_{h, 0,0}\right. \\
& +\sum_{i=1}^{j} w\left(\left(x^{\alpha}\right)_{k, l, j},\left(x^{\beta}\right)_{k, l, j},\left(x^{\gamma}\right)_{k, l, j},\left(x^{\delta}\right)_{k, 0, i}\right) \Delta_{i}\left(x^{\delta}\right)_{k, 0, i} \\
& \left.+\sum_{m=1}^{l} w\left(\left(x^{\alpha}\right)_{k, l, j},\left(x^{\beta}\right)_{k, l, j},\left(x^{\gamma}\right)_{k, l, j},\left(x^{\delta}\right)_{k, m, j}\right) \Delta_{m}\left(x^{\delta}\right)_{k, m, j}\right] \frac{1}{3 !} \varepsilon_{\alpha \beta \gamma \delta}\left(\Delta^{3} V^{\alpha \beta \gamma}\right)_{k, l, j} \\
& +\left[\sum_{h=1}^{k} w\left(\left(x^{\alpha}\right)_{k, j, l},\left(x^{\beta}\right)_{k, j, l},\left(x^{\gamma}\right)_{k, j, l},\left(x^{\delta}\right)_{h, 0,0}\right) \Delta_{h}\left(x^{\delta}\right)_{h, 0,0}\right.
\end{aligned}
$$




$$
\begin{aligned}
& +\sum_{i=1}^{j} w\left(\left(x^{\alpha}\right)_{k, j, l},\left(x^{\beta}\right)_{k, j, l}\left(x^{\gamma}\right)_{k, j, l},\left(x^{\delta}\right)_{k, i, 0}\right) \Delta_{i}\left(x^{\delta}\right)_{k, i, 0} \\
& \left.+\sum_{m=1}^{l} w\left(\left(x^{\alpha}\right)_{k, j, l},\left(x^{\beta}\right)_{k, j, l}\left(x^{\gamma}\right)_{k, j, l},\left(x^{\delta}\right)_{k, j, m}\right) \Delta_{m}\left(x^{\delta}\right)_{k, j, m}\right] \\
& \left.\times \frac{1}{3 !} \varepsilon_{\alpha \beta \gamma \delta}\left(\Delta^{3} V^{\alpha \beta \gamma}\right)_{k, j, l}\right\}
\end{aligned}
$$

where the indices are summed over $\alpha, \beta, \gamma, \delta, \mu=0,1,2,3$. In order for (4.115) to hold for any value of integral variables, the following $3 \times 3$ ! = 18 kinds of formulae in three categories are required.

1. The formulae for $\alpha, \beta, \gamma, \delta=0,1,2,3$ and $h=1,2, \ldots, k$,

$$
\begin{aligned}
& \lim _{\Delta_{h}\left(x^{\delta}\right)_{0, h, 0} \rightarrow 0} \frac{\Delta X^{\mu}\left[\Delta_{h}\left(x^{\delta}\right)_{0, h, 0}\right]}{\Delta_{h}\left(x^{\delta}\right)_{0, h, 0}} \frac{1}{3 !} \varepsilon_{\alpha \beta \gamma \mu} \\
& =w\left(\left(x^{\alpha}\right)_{j, k, l},\left(x^{\beta}\right)_{j, k, l},\left(x^{\gamma}\right)_{j, k, l}\left(x^{\delta}\right)_{0, h, 0}\right) \frac{1}{4 !} \varepsilon_{\alpha \beta \gamma \delta}, \\
& \lim _{\Delta_{h}\left(x^{\delta}\right)_{0,0, h} \rightarrow 0} \frac{\Delta X^{\mu}\left[\Delta_{h}\left(x^{\delta}\right)_{0,0, h}\right]}{\Delta_{h}\left(x^{\delta}\right)_{0,0, h}} \frac{1}{3 !} \varepsilon_{\alpha \beta \gamma \mu} \\
& =w\left(\left(x^{\alpha}\right)_{j, l, k^{\prime}}\left(x^{\beta}\right)_{j, l, k},\left(x^{\gamma}\right)_{j, l, k},\left(x^{\delta}\right)_{0,0, h}\right) \frac{1}{4 !} \varepsilon_{\alpha \beta \gamma \delta}, \\
& \lim _{\Delta_{h}\left(x^{\delta}\right)_{0,0, h} \rightarrow 0} \frac{\Delta X^{\mu}\left[\Delta_{h}\left(x^{\delta}\right)_{0,0, h}\right]}{\Delta_{h}\left(x^{\delta}\right)_{0,0, h}} \frac{1}{3 !} \varepsilon_{\alpha \beta \gamma \mu} \\
& =w\left(\left(x^{\alpha}\right)_{l, j, k^{\prime}}\left(x^{\beta}\right)_{l, j, k},\left(x^{\gamma}\right)_{l, j, k^{\prime}},\left(x^{\delta}\right)_{0,0, h}\right) \frac{1}{4 !} \varepsilon_{\alpha \beta \gamma \delta}, \\
& \lim _{\Delta_{h}\left(x^{\delta}\right)_{0, h, 0} \rightarrow 0} \frac{\Delta X^{\mu}\left[\Delta_{h}\left(x^{\delta}\right)_{0, h, 0}\right]}{\Delta_{h}\left(x^{\delta}\right)_{0, h, 0}} \frac{1}{3 !} \varepsilon_{\alpha \beta \gamma \mu} \\
& =w\left(\left(x^{\alpha}\right)_{l, k, j}\left(x^{\beta}\right)_{l, k, j}\left(x^{\gamma}\right)_{l, k, j}\left(x^{\delta}\right)_{0, h, 0}\right) \frac{1}{4 !} \varepsilon_{\alpha \beta \gamma \delta}, \\
& \lim _{\Delta_{h}\left(x^{\delta}\right)_{h, 0,0} \rightarrow 0} \frac{\Delta X^{\mu}\left[\Delta_{h}\left(x^{\delta}\right)_{h, 0,0}\right]}{\Delta_{h}\left(x^{\delta}\right)_{h, 0,0}} \frac{1}{3 !} \varepsilon_{\alpha \beta \gamma \mu} \\
& =w\left(\left(x^{\alpha}\right)_{k, l, j},\left(x^{\beta}\right)_{k, l, j^{\prime}},\left(x^{\gamma}\right)_{k, l, j},\left(x^{\delta}\right)_{h, 0,0}\right) \frac{1}{4 !} \varepsilon_{\alpha \beta \gamma \delta}, \\
& \lim _{\Delta_{h}\left(x^{\delta}\right)_{h, 0,0} \rightarrow 0} \frac{\Delta X^{\mu}\left[\Delta_{h}\left(x^{\delta}\right)_{h, 0,0}\right]}{\Delta_{h}\left(x^{\delta}\right)_{h, 0,0}} \frac{1}{3 !} \varepsilon_{\alpha \beta \gamma \mu} \\
& =w\left(\left(x^{\alpha}\right)_{k, j, l},\left(x^{\beta}\right)_{k, j, l},\left(x^{\gamma}\right)_{k, j, l},\left(x^{\delta}\right)_{h, 0,0}\right) \frac{1}{4 !} \varepsilon_{\alpha \beta \gamma \delta},
\end{aligned}
$$

where the index is summed over $\mu=0,1,2,3$.

2. The formulae for $\alpha, \beta, \gamma, \delta=0,1,2,3$ and $i=1,2, \ldots, j$ and $k=1,2, \ldots, n$,

$$
\begin{aligned}
& \lim _{\Delta_{i}\left(x^{\delta}\right)_{i, k, 0} \rightarrow 0} \frac{\Delta X^{\mu}\left[\Delta_{i}\left(x^{\delta}\right)_{i, k, 0}\right]}{\Delta_{i}\left(x^{\delta}\right)_{i, k, 0}} \frac{1}{3 !} \varepsilon_{\alpha \beta \gamma \mu} \\
& =w\left(\left(x^{\alpha}\right)_{j, k, l}\left(x^{\beta}\right)_{j, k, l}\left(x^{\gamma}\right)_{j, k, l},\left(x^{\delta}\right)_{i, k, 0}\right) \frac{1}{4 !} \varepsilon_{\alpha \beta \gamma \delta},
\end{aligned}
$$




$$
\begin{aligned}
& \lim _{\Delta_{i}\left(x^{\delta}\right)_{k, 0, i} \rightarrow 0} \frac{\Delta X^{\mu}\left[\Delta_{i}\left(x^{\delta}\right)_{k, 0, i}\right]}{\Delta_{i}\left(x^{\delta}\right)_{k, 0, i}} \frac{1}{3 !} \varepsilon_{\alpha \beta \gamma \mu} \\
& =w\left(\left(x^{\alpha}\right)_{k, l, j},\left(x^{\beta}\right)_{k, l, j},\left(x^{\gamma}\right)_{k, l, j},\left(x^{\delta}\right)_{k, 0, i}\right) \frac{1}{4 !} \varepsilon_{\alpha \beta \gamma \delta}, \\
& \lim _{\Delta_{i}\left(x^{\delta}\right)_{k, i, 0} \rightarrow 0} \frac{\Delta X^{\mu}\left[\Delta_{i}\left(x^{\delta}\right)_{k, i, 0}\right]}{\Delta_{i}\left(x^{\delta}\right)_{k, i, 0}} \frac{1}{3 !} \varepsilon_{\alpha \beta \gamma \mu} \\
& =w\left(\left(x^{\alpha}\right)_{k, j, l},\left(x^{\beta}\right)_{k, j, l},\left(x^{\gamma}\right)_{k, j, l},\left(x^{\delta}\right)_{k, i, 0}\right) \frac{1}{4 !} \varepsilon_{\alpha \beta \gamma \delta},
\end{aligned}
$$

where the index is summed over $\mu=0,1,2,3$.

3. The formulae for $\alpha, \beta, \gamma, \delta=0,1,2,3$ and $m=1,2, \ldots, l$ and $j=1,2, \ldots, k$ and $k=$ $1,2, \ldots, n$,

$$
\begin{aligned}
& \lim _{\Delta_{m}\left(x^{\delta}\right)_{j, k, m} \rightarrow 0} \frac{\Delta X^{\mu}\left[\Delta_{m}\left(x^{\delta}\right)_{j, k, m}\right]}{\Delta_{m}\left(x^{\delta}\right)_{j, k, m}} \frac{1}{3 !} \varepsilon_{\alpha \beta \gamma \mu} \\
& =w\left(\left(x^{\alpha}\right)_{j, k, l},\left(x^{\beta}\right)_{j, k, l}\left(x^{\gamma}\right)_{j, k, l},\left(x^{\delta}\right)_{j, k, m}\right) \frac{1}{4 !} \varepsilon_{\alpha \beta \gamma \delta}, \\
& \lim _{\Delta_{m}\left(x^{\delta}\right)_{j, m, k \rightarrow 0}} \frac{\Delta X^{\mu}\left[\Delta_{m}\left(x^{\delta}\right)_{j, m, k}\right]}{\Delta_{m}\left(x^{\delta}\right)_{j, m, k}} \frac{1}{3 !} \varepsilon_{\alpha \beta \gamma \mu} \\
& =w\left(\left(x^{\alpha}\right)_{j, l, k},\left(x^{\beta}\right)_{j, l, k}\left(x^{\gamma}\right)_{j, l, k},\left(x^{\delta}\right)_{j, m, k}\right) \frac{1}{4 !} \varepsilon_{\alpha \beta \gamma \delta}, \\
& =\lim _{\Delta_{m}\left(x^{\delta}\right)_{m, j, k} \rightarrow 0} \frac{\Delta X^{\mu}\left[\Delta_{m}\left(x^{\delta}\right)_{m, j, k}\right]}{\Delta_{j}\left(x^{\delta}\right)_{m, j, k}} \frac{1}{3 !} \varepsilon_{\alpha \beta \gamma \mu} \\
& =w\left(\left(x^{\alpha}\right)_{l, j, k},\left(x^{\beta}\right)_{l, j, k}\left(x^{\gamma}\right)_{l, j, k},\left(x^{\delta}\right)_{m, j, k}\right) \frac{1}{4 !} \varepsilon_{\alpha \beta \gamma \delta}, \\
& \lim _{\Delta_{m}\left(x^{\delta}\right)_{m, k, j} \rightarrow 0} \frac{\Delta X^{\mu}\left[\Delta_{m}\left(x^{\delta}\right)_{m, k, j}\right]}{\Delta_{m}\left(x^{\delta}\right)_{m, k, j}} \frac{1}{3 !} \varepsilon_{\alpha \beta \gamma \mu} \\
& =w\left(\left(x^{\alpha}\right)_{l, k, j}\left(x^{\beta}\right)_{l, k, j},\left(x^{\gamma}\right)_{l, k, j},\left(x^{\delta}\right)_{m, k, j}\right) \frac{1}{4 !} \varepsilon_{\alpha \beta \gamma \delta}, \\
& \lim _{\Delta_{m}\left(x^{\delta}\right)_{k, m, j} \rightarrow 0} \frac{\Delta X^{\mu}\left[\Delta_{m}\left(x^{\delta}\right)_{k, m, j}\right]}{\Delta_{m}\left(x^{\delta}\right)_{k, m, j}} \frac{1}{3 !} \varepsilon_{\alpha \beta \gamma \mu} \\
& =w\left(\left(x^{\alpha}\right)_{k, l, j},\left(x^{\beta}\right)_{k, l, j},\left(x^{\gamma}\right)_{k, l, j},\left(x^{\delta}\right)_{k, m, j}\right) \frac{1}{4 !} \varepsilon_{\alpha \beta \gamma \delta},
\end{aligned}
$$




$$
\begin{aligned}
& \lim _{\Delta_{m}\left(x^{\delta}\right)_{k, j, m} \rightarrow 0} \frac{\Delta X^{\mu}\left[\Delta_{m}\left(x^{\delta}\right)_{k, j, m}\right]}{\Delta_{m}\left(x^{\delta}\right)_{k, j, m}} \frac{1}{3 !} \varepsilon_{\alpha \beta \gamma \mu} \\
& =w\left(\left(x^{\alpha}\right)_{k, j, l},\left(x^{\beta}\right)_{k, j, l}\left(x^{\gamma}\right)_{k, j, l}\left(x^{\delta}\right)_{k, j, m}\right) \frac{1}{4 !} \varepsilon_{\alpha \beta \gamma \delta},
\end{aligned}
$$

where the index is summed over $\mu=0,1,2,3$.

In the 4D time-space, $\frac{1}{3 !} \varepsilon_{\alpha \beta \gamma \mu} \varepsilon^{\alpha \beta \gamma \delta}=\delta_{\mu}^{\delta}$ holds for $\delta, \mu=0,1,2,3$, where the indices are summed over $\alpha, \beta, \gamma=0,1,2,3$. We therefore obtain $\frac{1}{4 !} \varepsilon_{\alpha \beta \gamma \delta} \varepsilon^{\alpha \beta \gamma \delta}=1$, where the indices are summed over $\alpha, \beta, \gamma, \delta=0,1,2,3$. Multiplying by $\varepsilon^{\alpha \beta \gamma \delta}$ both sides of the 18 kinds of formulae (4.116), (4.117), (4.118), (4.119), (4.120), (4.121), (4.122), (4.123), (4.124), (4.125), (4.126), (4.127), (4.128), (4.129), (4.130), (4.131), (4.132), and (4.133), they are reduced to the differential equation (4.113).

\section{Appendix 1: Calculations for Example 1 in detail}

The value of $\lim _{n \rightarrow \infty} \sum_{k=1}^{n} x_{k} y_{k}^{2} \Delta y_{k}$ in (2.43) is

$$
\begin{aligned}
& \lim _{n \rightarrow \infty} \sum_{k=1}^{n} x_{k} y_{k}^{2} \Delta y_{k} \\
& =a b^{3} \lim _{n \rightarrow \infty} \sum_{k=1}^{n} \cos \varphi_{k} \sin ^{2} \varphi_{k}\left(\sin \varphi_{k}-\sin \varphi_{k-1}\right) \\
& =a b^{3} \lim _{n \rightarrow \infty} \sum_{k=1}^{n} \cos \left(k \frac{2 \pi}{n}\right) \sin ^{2}\left(k \frac{2 \pi}{n}\right)\left\{\sin \left[k \frac{2 \pi}{n}\right]-\sin \left[(k-1) \frac{2 \pi}{n}\right]\right\} \\
& =\frac{1}{4} \pi a b^{3} .
\end{aligned}
$$

The value of $\lim _{n \rightarrow \infty} \sum_{k=1}^{n} x_{k}^{2} y_{k} \Delta x_{k}$ in (2.43) is

$$
\begin{aligned}
& \lim _{n \rightarrow \infty} \sum_{k=1}^{n} x_{k}^{2} y_{k} \Delta x_{k} \\
& \quad=a^{3} b \lim _{n \rightarrow \infty} \sum_{k=1}^{n} \cos ^{2} \varphi_{k} \sin \varphi_{k}\left(\cos \varphi_{k}-\cos \varphi_{k-1}\right) \\
& \quad=a^{3} b \lim _{n \rightarrow \infty} \sum_{k=1}^{n} \cos ^{2}\left(k \frac{2 \pi}{n}\right) \sin \left(k \frac{2 \pi}{n}\right)\left\{\cos \left[k \frac{2 \pi}{n}\right]-\cos \left[(k-1) \frac{2 \pi}{n}\right]\right\} \\
& \quad=-\frac{1}{4} \pi a^{3} b .
\end{aligned}
$$

The value of $\lim _{n \rightarrow \infty} \sum_{k=1}^{n} \sum_{j=1}^{k} x_{j}^{2} \Delta x_{j} \Delta y_{k}$ in (2.44) is

$$
\begin{aligned}
& \lim _{n \rightarrow \infty} \sum_{k=1}^{n} \sum_{j=1}^{k} x_{j}^{2} \Delta x_{j} \Delta y_{k} \\
& \quad=a^{3} b \lim _{n \rightarrow \infty} \sum_{k=1}^{n} \sum_{j=1}^{k} \cos ^{2} \varphi_{j}\left(\cos \varphi_{j}-\cos \varphi_{j-1}\right)\left(\sin \varphi_{k}-\sin \varphi_{k-1}\right)
\end{aligned}
$$




$$
\begin{aligned}
= & a^{3} b \lim _{n \rightarrow \infty} \sum_{k=1}^{n} \sum_{j=1}^{k} \cos ^{2}\left(j \frac{2 \pi}{n}\right)\left\{\cos \left[j \frac{2 \pi}{n}\right]-\cos \left[(j-1) \frac{2 \pi}{n}\right]\right\} \\
& \times\left\{\sin \left[k \frac{2 \pi}{n}\right]-\sin \left[(k-1) \frac{2 \pi}{n}\right]\right\} \\
= & \frac{1}{4} \pi a^{3} b .
\end{aligned}
$$

The value of $\lim _{n \rightarrow \infty} \sum_{k=1}^{n} \sum_{j=1}^{k} y_{k}^{2} \Delta x_{j} \Delta y_{k}$ in (2.44) is

$$
\begin{aligned}
\lim _{n \rightarrow \infty} \sum_{k=1}^{n} \sum_{j=1}^{k} y_{k}^{2} \Delta x_{j} \Delta y_{k} \\
=a b^{3} \lim _{n \rightarrow \infty} \sum_{k=1}^{n} \sum_{j=1}^{k} \sin ^{2} \varphi_{k}\left(\cos \varphi_{j}-\cos \varphi_{j-1}\right)\left(\sin \varphi_{k}-\sin \varphi_{k-1}\right) \\
=a b^{3} \lim _{n \rightarrow \infty} \sum_{k=1}^{n} \sum_{j=1}^{k} \sin ^{2}\left(k \frac{2 \pi}{n}\right)\left\{\cos \left[j \frac{2 \pi}{n}\right]-\cos \left[(j-1) \frac{2 \pi}{n}\right]\right\} \\
\quad \times\left\{\sin \left[k \frac{2 \pi}{n}\right]-\sin \left[(k-1) \frac{2 \pi}{n}\right]\right\} \\
=\frac{1}{4} \pi a b^{3} .
\end{aligned}
$$

The value of $\lim _{n \rightarrow \infty} \sum_{k=1}^{n} \sum_{j=1}^{k} x_{k}^{2} \Delta x_{k} \Delta y_{j}$ in (2.44) is

$$
\begin{aligned}
\lim _{n \rightarrow \infty} \sum_{k=1}^{n} \sum_{j=1}^{k} x_{k}^{2} \Delta x_{k} \Delta y_{j} \\
=a^{3} b \lim _{n \rightarrow \infty} \sum_{k=1}^{n} \sum_{j=1}^{k} \cos ^{2} \varphi_{k}\left(\cos \varphi_{k}-\cos \varphi_{k-1}\right)\left(\sin \varphi_{j}-\sin \varphi_{j-1}\right) \\
=a^{3} b \lim _{n \rightarrow \infty} \sum_{k=1}^{n} \sum_{j=1}^{k} \cos ^{2}\left(k \frac{2 \pi}{n}\right)\left\{\cos \left[k \frac{2 \pi}{n}\right]-\cos \left[(k-1) \frac{2 \pi}{n}\right]\right\} \\
\quad \times\left\{\sin \left[j \frac{2 \pi}{n}\right]-\sin \left[(j-1) \frac{2 \pi}{n}\right]\right\} \\
=-\frac{1}{4} \pi a^{3} b .
\end{aligned}
$$

The value of $\lim _{n \rightarrow \infty} \sum_{k=1}^{n} \sum_{j=1}^{k} y_{j}^{2} \Delta x_{k} \Delta y_{j}$ in (2.44) is

$$
\begin{aligned}
& \lim _{n \rightarrow \infty} \sum_{k=1}^{n} \sum_{j=1}^{k} y_{j}^{2} \Delta x_{k} \Delta y_{j} \\
& \quad=a b^{3} \lim _{n \rightarrow \infty} \sum_{k=1}^{n} \sum_{j=1}^{k} \sin ^{2} \varphi_{j}\left(\cos \varphi_{k}-\cos \varphi_{k-1}\right)\left(\sin \varphi_{j}-\sin \varphi_{j-1}\right)
\end{aligned}
$$




$$
\begin{aligned}
= & a b^{3} \lim _{n \rightarrow \infty} \sum_{k=1}^{n} \sum_{j=1}^{k} \sin ^{2}\left(j \frac{2 \pi}{n}\right)\left\{\cos \left[k \frac{2 \pi}{n}\right]-\cos \left[(k-1) \frac{2 \pi}{n}\right]\right\} \\
& \times\left\{\sin \left[j \frac{2 \pi}{n}\right]-\sin \left[(j-1) \frac{2 \pi}{n}\right]\right\} \\
= & -\frac{1}{4} \pi a b^{3} .
\end{aligned}
$$

\section{Appendix 2: Calculations for Example 2 in detail}

1. Calculation of (3.62) for Example 2 in detail.

Since $\Delta_{k} z_{j-1, k}=0$ and $\Delta_{k} z_{j, k}=0$, the value of $\lim _{n \rightarrow \infty} \sum_{k=1}^{n} \sum_{j=1}^{k} X_{j, k}\left(\Delta^{2} \sigma_{x}\right)_{j, k}$ is

$$
\begin{aligned}
& \lim _{n \rightarrow \infty} \sum_{k=1}^{n} \sum_{j=1}^{k} X_{j, k}\left(\Delta^{2} \sigma_{x}\right)_{j, k} \\
& =\frac{1}{4} \lim _{n \rightarrow \infty} \sum_{k=1}^{n} \sum_{j=1}^{k} x_{j, k}\left(y_{j, k}^{2}+z_{j, k}^{2}\right)\left(\Delta_{j} y_{j, k} \Delta_{k} z_{j-1, k}+\Delta_{k} z_{j, k} \Delta_{j} y_{j, k-1}\right) \\
& -\frac{1}{4} \lim _{n \rightarrow \infty} \sum_{k=1}^{n} \sum_{j=1}^{k} x_{j, k}\left(y_{j, k}^{2}+z_{j, k}^{2}\right)\left(\Delta_{j} z_{j, k} \Delta_{k} y_{j-1, k}+\Delta_{k} y_{j, k} \Delta_{j} z_{j, k-1}\right) \\
& =0-\frac{a^{5}}{4} \lim _{n \rightarrow \infty} \sum_{k=1}^{n} \sum_{j=1}^{k} \sin \theta_{j} \cos \varphi_{k}\left(\sin ^{2} \theta_{j} \sin ^{2} \varphi_{k}+\cos ^{2} \theta_{j}\right) \\
& \times\left(\cos \theta_{j}-\cos \theta_{j-1}\right)\left(\sin \theta_{j-1}+\sin \theta_{j}\right)\left(\sin \varphi_{k}-\sin \varphi_{k-1}\right) \\
& =-\frac{a^{5}}{4} \lim _{n \rightarrow \infty} \sum_{k=1}^{n} \sum_{j=1}^{k} \sin \left(\frac{j}{n} \pi\right) \cos \left(2 \frac{k}{n} \pi\right)\left[\sin ^{2}\left(\frac{j}{n} \pi\right) \sin ^{2}\left(2 \frac{k}{n} \pi\right)+\cos ^{2}\left(\frac{j}{n} \pi\right)\right] \\
& \times\left[\cos \left(\frac{j}{n} \pi\right)-\cos \left(\frac{j-1}{n} \pi\right)\right]\left[\sin \left(\frac{j-1}{n} \pi\right)+\sin \left(\frac{j}{n} \pi\right)\right] \\
& \times\left[\sin \left(2 \frac{k}{n} \pi\right)-\sin \left(2 \frac{k-1}{n} \pi\right)\right] \\
& =\frac{2}{15} \pi a^{5} \text {. }
\end{aligned}
$$

Since $\Delta_{j} z_{k-1, j}=0$ and $\Delta_{j} z_{k, j}=0$, the value of $\lim _{n \rightarrow \infty} \sum_{k=1}^{n} \sum_{j=1}^{k} X_{k, j}\left(\Delta^{2} \sigma_{x}\right)_{k, j}$ is

$$
\begin{aligned}
\lim _{n \rightarrow \infty} \sum_{k=1}^{n} \sum_{j=1}^{k} X_{k, j}\left(\Delta^{2} \sigma_{x}\right)_{k, j} \\
=\frac{1}{4} \lim _{n \rightarrow \infty} \sum_{k=1}^{n} \sum_{j=1}^{k} x_{k, j}\left(y_{k, j}^{2}+z_{k, j}^{2}\right)\left(\Delta_{k} y_{k, j} \Delta_{j} z_{k-1, j}+\Delta_{j} z_{k, j} \Delta_{k} y_{k, j-1}\right) \\
\quad-\frac{1}{4} \lim _{n \rightarrow \infty} \sum_{k=1}^{n} \sum_{j=1}^{k} x_{k, j}\left(y_{k, j}^{2}+z_{k, j}^{2}\right)\left(\Delta_{k} z_{k, j} \Delta_{j} y_{k-1, j}+\Delta_{j} y_{k, j} \Delta_{k} z_{k, j-1}\right) \\
=0-\frac{a^{5}}{4} \lim _{n \rightarrow \infty} \sum_{k=1}^{n} \sum_{j=1}^{k} \sin \theta_{k} \cos \varphi_{j}\left(\sin ^{2} \theta_{k} \sin ^{2} \varphi_{j}+\cos ^{2} \theta_{k}\right)
\end{aligned}
$$




$$
\begin{aligned}
& \times\left(\cos \theta_{k}-\cos \theta_{k-1}\right)\left(\sin \theta_{k-1}+\sin \theta_{k}\right)\left(\sin \varphi_{j}-\sin \varphi_{j-1}\right) \\
= & -\frac{a^{5}}{4} \lim _{n \rightarrow \infty} \sum_{k=1}^{n} \sum_{j=1}^{k} \sin \left(\frac{k}{n} \pi\right) \cos \left(2 \frac{j}{n} \pi\right)\left[\sin ^{2}\left(\frac{k}{n} \pi\right) \sin ^{2}\left(2 \frac{j}{n} \pi\right)+\cos ^{2}\left(\frac{k}{n} \pi\right)\right] \\
& \times\left[\cos \left(\frac{k}{n} \pi\right)-\cos \left(\frac{k-1}{n} \pi\right)\right]\left[\sin \left(\frac{k-1}{n} \pi\right)+\sin \left(\frac{k}{n} \pi\right)\right] \\
& \times\left[\sin \left(2 \frac{j}{n} \pi\right)-\sin \left(2 \frac{j-1}{n} \pi\right)\right] \\
= & \frac{2}{15} \pi a^{5} .
\end{aligned}
$$

Substituting (B.1) and (B.2) into (3.62), we obtain

$$
\iint_{\partial D} X d^{2} \sigma_{x}=\frac{4}{15} \pi a^{5}
$$

2. Calculation of (3.63) for Example 2 in detail.

Since $\Delta_{k} z_{j-1, k}=0$ and $\Delta_{k} z_{j, k}=0$, the value of $\lim _{n \rightarrow \infty} \sum_{k=1}^{n} \sum_{j=1}^{k} Y_{j, k}\left(\Delta^{2} \sigma_{y}\right)_{j, k}$ is

$$
\begin{aligned}
\lim _{n \rightarrow \infty} \sum_{k=1}^{n} \sum_{j=1}^{k} Y_{j, k}\left(\Delta^{2} \sigma_{y}\right)_{j, k} \\
=\frac{1}{4} \lim _{n \rightarrow \infty} \sum_{k=1}^{n} \sum_{j=1}^{k} y_{j, k}\left(z_{j, k}^{2}+x_{j, k}^{2}\right)\left(\Delta_{j} z_{j, k} \Delta_{k} x_{j-1, k}+\Delta_{k} x_{j, k} \Delta_{j} z_{j, k-1}\right) \\
-\frac{1}{4} \lim _{n \rightarrow \infty} \sum_{k=1}^{n} \sum_{j=1}^{k} y_{j, k}\left(z_{j, k}^{2}+x_{j, k}^{2}\right)\left(\Delta_{j} x_{j, k} \Delta_{k} z_{j-1, k}+\Delta_{k} z_{j, k} \Delta_{j} x_{j, k-1}\right) \\
=\frac{a^{5}}{4} \lim _{n \rightarrow \infty} \sum_{k=1}^{n} \sum_{j=1}^{k} \sin \theta_{j} \sin \varphi_{k}\left(\cos ^{2} \theta_{j}+\sin ^{2} \theta_{j} \cos ^{2} \varphi_{k}\right) \\
\times\left(\cos \theta_{j}-\cos \theta_{j-1}\right)\left(\sin \theta_{j-1}+\sin \theta_{j}\right)\left(\cos _{\varphi_{k}}-\cos _{k-1}\right)+0 \\
=\frac{a^{5}}{4} \lim _{n \rightarrow \infty} \sum_{k=1}^{n} \sum_{j=1}^{k} \sin \left(\frac{j}{n} \pi\right) \sin \left(2 \frac{k}{n} \pi\right)\left[\cos ^{2}\left(\frac{j}{n} \pi\right)+\sin ^{2}\left(\frac{j}{n} \pi\right) \cos ^{2}\left(2 \frac{k}{n} \pi\right)\right] \\
\quad \times\left[\cos \left(\frac{j}{n} \pi\right)-\cos \left(\frac{j-1}{n} \pi\right)\right]\left[\sin \left(\frac{j-1}{n} \pi\right)+\sin \left(\frac{j}{n} \pi\right)\right] \\
\quad \times\left[\cos \left(2 \frac{k}{n} \pi\right)-\cos \left(2 \frac{k-1}{n} \pi\right)\right] \\
=\frac{2}{15} \pi a^{5} .
\end{aligned}
$$

Since $\Delta_{j} z_{k-1, j}=0$ and $\Delta_{j} z_{k, j}=0$, the value of $\lim _{n \rightarrow \infty} \sum_{k=1}^{n} \sum_{j=1}^{k} Y_{k, j}\left(\Delta^{2} \sigma_{y}\right)_{k, j}$ is

$$
\begin{aligned}
& \lim _{n \rightarrow \infty} \sum_{k=1}^{n} \sum_{j=1}^{k} Y_{k, j}\left(\Delta^{2} \sigma_{y}\right)_{k, j} \\
& =\frac{1}{4} \lim _{n \rightarrow \infty} \sum_{k=1}^{n} \sum_{j=1}^{k} y_{k, j}\left(z_{k, j}^{2}+x_{k, j}^{2}\right)\left(\Delta_{k} z_{k, j} \Delta_{j} x_{k-1, j}+\Delta_{j} x_{k, j} \Delta_{k} z_{k, j-1}\right)
\end{aligned}
$$




$$
\begin{aligned}
& -\frac{1}{4} \lim _{n \rightarrow \infty} \sum_{k=1}^{n} \sum_{j=1}^{k} y_{k, j}\left(z_{k, j}^{2}+x_{k, j}^{2}\right)\left(\Delta_{k} x_{k, j} \Delta_{j} z_{k-1, j}+\Delta_{j} z_{k, j} \Delta_{k} x_{k, j-1}\right) \\
= & \frac{a^{5}}{4} \lim _{n \rightarrow \infty} \sum_{k=1}^{n} \sum_{j=1}^{k} \sin \theta_{k} \sin \varphi_{j}\left(\cos ^{2} \theta_{k}+\sin ^{2} \theta_{k} \cos ^{2} \varphi_{j}\right) \\
& \times\left(\cos \theta_{k}-\cos \theta_{k-1}\right)\left(\sin \theta_{k-1}+\sin \theta_{k}\right)\left(\cos \varphi_{j}-\cos \varphi_{j-1}\right)+0 \\
= & \frac{a^{5}}{4} \lim _{n \rightarrow \infty} \sum_{k=1}^{n} \sum_{j=1}^{k} \sin \left(\frac{k}{n} \pi\right) \sin \left(2 \frac{j}{n} \pi\right)\left[\cos ^{2}\left(\frac{k}{n} \pi\right)+\sin ^{2}\left(\frac{k}{n} \pi\right) \cos ^{2}\left(2 \frac{j}{n} \pi\right)\right] \\
& \times\left[\cos \left(\frac{k}{n} \pi\right)-\cos \left(\frac{k-1}{n} \pi\right)\right]\left[\sin \left(\frac{k-1}{n} \pi\right)+\sin \left(\frac{k}{n} \pi\right)\right] \\
& \times\left[\cos \left(2 \frac{j}{n} \pi\right)-\cos \left(2 \frac{j-1}{n} \pi\right)\right] \\
= & \frac{2}{15} \pi a^{5} .
\end{aligned}
$$

Substituting (B.4) and (B.5) into (3.63), we obtain

$$
\iint_{\partial D} Y d^{2} \sigma_{y}=\frac{4}{15} \pi a^{5}
$$

3. Calculation of (3.64) for Example 2 in detail.

The value of $\lim _{n \rightarrow \infty} \sum_{k=1}^{n} \sum_{j=1}^{k} Z_{j, k}\left(\Delta^{2} \sigma_{z}\right)_{j, k}$ is

$$
\begin{aligned}
\lim _{n \rightarrow \infty} \sum_{k=1}^{n} \sum_{j=1}^{k} Z_{j, k}\left(\Delta^{2} \sigma_{z}\right)_{j, k} \\
=\frac{1}{4} \lim _{n \rightarrow \infty} \sum_{k=1}^{n} \sum_{j=1}^{k} z_{j, k}\left(x_{j, k}^{2}+y_{j, k}^{2}\right)\left(\Delta_{j} x_{j, k} \Delta_{k} y_{j-1, k}+\Delta_{k} y_{j, k} \Delta_{j} x_{j, k-1}\right) \\
-\frac{1}{4} \lim _{n \rightarrow \infty} \sum_{k=1}^{n} \sum_{j=1}^{k} z_{j, k}\left(x_{j, k}^{2}+y_{j, k}^{2}\right)\left(\Delta_{j} y_{j, k} \Delta_{k} x_{j-1, k}+\Delta_{k} x_{j, k} \Delta_{j} y_{j, k-1}\right) \\
=\frac{a^{5}}{4} \lim _{n \rightarrow \infty} \sum_{k=1}^{n} \sum_{j=1}^{k} \cos \theta_{j} \sin ^{2} \theta_{j}\left(\sin \theta_{j}-\sin \theta_{j-1}\right)\left(\sin \varphi_{k}-\sin \varphi_{k-1}\right) \\
\quad \times\left(\cos \varphi_{k} \sin \theta_{j-1}+\cos \varphi_{k-1} \sin \theta_{j}\right) \\
-\frac{a^{5}}{4} \lim _{n \rightarrow \infty} \sum_{k=1}^{n} \sum_{j=1}^{k} \cos \theta_{j} \sin { }^{2} \theta_{j}\left(\sin \theta_{j}-\sin \theta_{j-1}\right)\left(\cos \varphi_{k}-\cos \varphi_{k-1}\right) \\
\quad \times\left(\sin \varphi_{k} \sin \theta_{j-1}+\sin \varphi_{k-1} \sin \theta_{j}\right) \\
=\frac{a^{5}}{4} \lim _{n \rightarrow \infty} \sum_{k=1}^{n} \sum_{j=1}^{k} \cos \left(\frac{j}{n} \pi\right) \sin ^{2}\left(\frac{j}{n} \pi\right) \\
\quad \times\left[\sin \left(\frac{j}{n} \pi\right)-\sin \left(\frac{j-1}{n} \pi\right)\right]\left[\sin \left(2 \frac{k}{n} \pi\right)-\sin \left(2 \frac{k-1}{n} \pi\right)\right]
\end{aligned}
$$




$$
\begin{aligned}
\times & {\left[\cos \left(2 \frac{k}{n} \pi\right) \sin \left(\frac{j-1}{n} \pi\right)+\cos \left(2 \frac{k-1}{n} \pi\right) \sin \left(\frac{j}{n} \pi\right)\right] } \\
- & \frac{a^{5}}{4} \lim _{n \rightarrow \infty} \sum_{k=1}^{n} \sum_{j=1}^{k} \cos \left(\frac{j}{n} \pi\right) \sin ^{2}\left(\frac{j}{n} \pi\right) \\
\times & {\left[\sin \left(\frac{j}{n} \pi\right)-\sin \left(\frac{j-1}{n} \pi\right)\right]\left[\cos \left(2 \frac{k}{n} \pi\right)-\cos \left(2 \frac{k-1}{n} \pi\right)\right] } \\
& \times\left[\sin \left(2 \frac{k}{n} \pi\right) \sin \left(\frac{j-1}{n} \pi\right)+\sin \left(2 \frac{k-1}{n} \pi\right) \sin \left(\frac{j}{n} \pi\right)\right] \\
= & \frac{1}{15} \pi a^{5}+\frac{1}{15} \pi a^{5} \\
= & \frac{2}{15} \pi a^{5} .
\end{aligned}
$$

The value of $\lim _{n \rightarrow \infty} \sum_{k=1}^{n} \sum_{j=1}^{k} Z_{k, j}\left(\Delta^{2} \sigma_{z}\right)_{k, j}$ is

$$
\begin{aligned}
& \lim _{n \rightarrow \infty} \sum_{k=1}^{n} \sum_{j=1}^{k} Z_{k, j}\left(\Delta^{2} \sigma_{z}\right)_{k, j} \\
& =\frac{1}{4} \lim _{n \rightarrow \infty} \sum_{k=1}^{n} \sum_{j=1}^{k} z_{k, j}\left(x_{k, j}^{2}+y_{k, j}^{2}\right)\left(\Delta_{k} x_{k, j} \Delta_{j} y_{k-1, j}+\Delta_{j} y_{k, j} \Delta_{k} x_{k, j-1}\right) \\
& -\frac{1}{4} \lim _{n \rightarrow \infty} \sum_{k=1}^{n} \sum_{j=1}^{k} z_{k, j}\left(x_{k, j}^{2}+y_{k, j}^{2}\right)\left(\Delta_{k} y_{k, j} \Delta_{j} x_{k-1, j}+\Delta_{j} x_{k, j} \Delta_{k} y_{k, j-1}\right) \\
& =\frac{a^{5}}{4} \lim _{n \rightarrow \infty} \sum_{k=1}^{n} \sum_{j=1}^{k} \cos \theta_{k} \sin ^{2} \theta_{k}\left(\sin \theta_{k}-\sin \theta_{k-1}\right)\left(\sin \varphi_{j}-\sin \varphi_{j-1}\right) \\
& \times\left(\cos \varphi_{j} \sin \theta_{k-1}+\cos \varphi_{j-1} \sin \theta_{k}\right) \\
& -\frac{a^{5}}{4} \lim _{n \rightarrow \infty} \sum_{k=1}^{n} \sum_{j=1}^{k} \cos \theta_{k} \sin ^{2} \theta_{k}\left(\sin \theta_{k}-\sin \theta_{k-1}\right)\left(\cos \varphi_{j}-\cos \varphi_{j-1}\right) \\
& \times\left(\sin \varphi_{j} \sin \theta_{k-1}+\sin \varphi_{j-1} \sin \theta_{k}\right) \\
& =\frac{a^{5}}{4} \lim _{n \rightarrow \infty} \sum_{k=1}^{n} \sum_{j=1}^{k} \cos \left(\frac{k}{n} \pi\right) \sin ^{2}\left(\frac{k}{n} \pi\right) \\
& \times\left[\sin \left(\frac{k}{n} \pi\right)-\sin \left(\frac{k-1}{n} \pi\right)\right]\left[\sin \left(2 \frac{j}{n} \pi\right)-\sin \left(2 \frac{j-1}{n} \pi\right)\right] \\
& \times\left[\cos \left(2 \frac{j}{n} \pi\right) \sin \left(\frac{k-1}{n} \pi\right)+\cos \left(2 \frac{j-1}{n} \pi\right) \sin \left(\frac{k}{n} \pi\right)\right] \\
& -\frac{a^{5}}{4} \lim _{n \rightarrow \infty} \sum_{k=1}^{n} \sum_{j=1}^{k} \cos \left(\frac{k}{n} \pi\right) \sin ^{2}\left(\frac{k}{n} \pi\right) \\
& \times\left[\sin \left(\frac{k}{n} \pi\right)-\sin \left(\frac{k-1}{n} \pi\right)\right]\left[\cos \left(2 \frac{j}{n} \pi\right)-\cos \left(2 \frac{j-1}{n} \pi\right)\right] \\
& \times\left[\sin \left(2 \frac{j}{n} \pi\right) \sin \left(\frac{k-1}{n} \pi\right)+\sin \left(2 \frac{j-1}{n} \pi\right) \sin \left(\frac{k}{n} \pi\right)\right]
\end{aligned}
$$




$$
\begin{aligned}
& =\frac{1}{15} \pi a^{5}+\frac{1}{15} \pi a^{5} \\
& =\frac{2}{15} \pi a^{5} .
\end{aligned}
$$

Substituting (B.7) and (B.8) into (3.64), we obtain

$$
\iint_{\partial D} Z d^{2} \sigma_{z}=\frac{4}{15} \pi a^{5}
$$

4. Calculation of (3.66) for Example 2 in detail.

Since $\Delta_{h} x_{0, h}=0$, the value of $\frac{1}{3} \lim _{n \rightarrow \infty} \sum_{k=1}^{n} \sum_{j=1}^{k} \sum_{h=1}^{k} q\left(x_{0, h}, y_{j, k}, z_{j, k}\right) \Delta_{h} x_{0, h}\left(\Delta^{2} \sigma_{x}\right)_{j, k}$ is

$$
\frac{1}{3} \lim _{n \rightarrow \infty} \sum_{k=1}^{n} \sum_{j=1}^{k} \sum_{h=1}^{k} q\left(x_{0, h}, y_{j, k}, z_{j, k}\right) \Delta_{h} x_{0, h}\left(\Delta^{2} \sigma_{x}\right)_{j, k}=0 .
$$

Since $\Delta_{k} z_{j-1, k}=0$ and $\Delta_{k} z_{j, k}=0$, the value of $\frac{1}{3} \lim _{n \rightarrow \infty} \sum_{k=1}^{n} \sum_{j=1}^{k} \sum_{i=1}^{j} q\left(x_{i, k}, y_{j, k}, z_{j, k}\right) \times$ $\Delta_{i} x_{i, k}\left(\Delta^{2} \sigma_{x}\right)_{j, k}$ is

$$
\begin{aligned}
& \frac{1}{3} \lim _{n \rightarrow \infty} \sum_{k=1}^{n} \sum_{j=1}^{k} \sum_{i=1}^{j} q\left(x_{i, k}, y_{j, k}, z_{j, k}\right) \Delta_{i} x_{i, k}\left(\Delta^{2} \sigma_{x}\right)_{j, k} \\
& =\frac{1}{6} \lim _{n \rightarrow \infty} \sum_{k=1}^{n} \sum_{j=1}^{k} \sum_{i=1}^{j}\left(x_{i, k}^{2}+y_{j, k}^{2}+z_{j, k}^{2}\right) \Delta_{i} x_{i, k}\left(\Delta_{j} y_{j, k} \Delta_{k} z_{j-1, k}+\Delta_{k} z_{j, k} \Delta_{j} y_{j, k-1}\right) \\
& -\frac{1}{6} \lim _{n \rightarrow \infty} \sum_{k=1}^{n} \sum_{j=1}^{k} \sum_{i=1}^{j}\left(x_{i, k}^{2}+y_{j, k}^{2}+z_{j, k}^{2}\right) \Delta_{i} x_{i, k}\left(\Delta_{j} z_{j, k} \Delta_{k} y_{j-1, k}+\Delta_{k} y_{j, k} \Delta_{j} z_{j, k-1}\right) \\
& =0-\frac{a^{5}}{6} \lim _{n \rightarrow \infty} \sum_{k=1}^{n} \sum_{j=1}^{k} \sum_{i=1}^{j}\left(\sin ^{2} \theta_{i} \cos ^{2} \varphi_{k}+\sin ^{2} \theta_{j} \sin ^{2} \varphi_{k}+\cos ^{2} \theta_{j}\right) \\
& \times\left(\sin \theta_{i}-\sin \theta_{i-1}\right) \cos \varphi_{k}\left(\cos \theta_{j}-\cos \theta_{j-1}\right) \\
& \times\left(\sin \theta_{j-1}+\sin \theta_{j}\right)\left(\sin \varphi_{k}-\sin \varphi_{k-1}\right) \\
& =-\frac{a^{5}}{6} \lim _{n \rightarrow \infty} \sum_{k=1}^{n} \sum_{j=1}^{k} \sum_{i=1}^{j}\left[\sin ^{2}\left(\frac{i}{n} \pi\right) \cos ^{2}\left(2 \frac{k}{n} \pi\right)\right. \\
& \left.+\sin ^{2}\left(\frac{j}{n} \pi\right) \sin ^{2}\left(2 \frac{k}{n} \pi\right)+\cos ^{2}\left(\frac{j}{n} \pi\right)\right] \\
& \times\left[\sin \left(\frac{i}{n} \pi\right)-\sin \left(\frac{i-1}{n} \pi\right)\right] \cos \left(2 \frac{k}{n} \pi\right) \\
& \times\left[\cos \left(\frac{j}{n} \pi\right)-\cos \left(\frac{j-1}{n} \pi\right)\right]\left[\sin \left(\frac{j-1}{n} \pi\right)+\sin \left(\frac{j}{n} \pi\right)\right] \\
& \times\left[\sin \left(2 \frac{k}{n} \pi\right)-\sin \left(2 \frac{k-1}{n} \pi\right)\right] \\
& =\frac{2}{15} \pi a^{5} \text {. }
\end{aligned}
$$


Since $\Delta_{j} z_{k-1, j}=0$ and $\Delta_{j} z_{k, j}=0$, the value of $\frac{1}{3} \lim _{n \rightarrow \infty} \sum_{k=1}^{n} \sum_{j=1}^{k} \sum_{h=1}^{k} q\left(x_{h, 0}, y_{k, j}, z_{k, j}\right) \times$ $\Delta_{h} x_{h, 0}\left(\Delta^{2} \sigma_{x}\right)_{k, j}$ is

$$
\begin{aligned}
\frac{1}{3} \lim _{n \rightarrow \infty} \sum_{k=1}^{n} \sum_{j=1}^{k} \sum_{h=1}^{k} q\left(x_{h, 0}, y_{k, j}, z_{k, j}\right) \Delta_{h} x_{h, 0}\left(\Delta^{2} \sigma_{x}\right)_{k, j} \\
=\frac{1}{6} \lim _{n \rightarrow \infty} \sum_{k=1}^{n} \sum_{j=1}^{k} \sum_{h=1}^{k}\left(x_{h, 0}^{2}+y_{k, j}^{2}+z_{k, j}^{2}\right) \Delta_{h} x_{h, 0}\left(\Delta_{k} y_{k, j} \Delta_{j} z_{k-1, j}+\Delta_{j} z_{k, j} \Delta_{k} y_{k, j-1}\right) \\
-\frac{1}{6} \lim _{n \rightarrow \infty} \sum_{k=1}^{n} \sum_{j=1}^{k} \sum_{h=1}^{k}\left(x_{h, 0}^{2}+y_{k, j}^{2}+z_{k, j}^{2}\right) \Delta_{h} x_{h, 0}\left(\Delta_{k} z_{k, j} \Delta_{j} y_{k-1, j}+\Delta_{j} y_{k, j} \Delta_{k} z_{k, j-1}\right) \\
=0-\frac{a^{5}}{6} \lim _{n \rightarrow \infty} \sum_{k=1}^{n} \sum_{j=1}^{k} \sum_{h=1}^{k}\left(\sin ^{2} \theta_{h}+\sin ^{2} \theta_{k} \sin ^{2} \varphi_{j}+\cos ^{2} \theta_{k}\right)\left(\sin \theta_{h}-\sin \theta_{h-1}\right) \\
\quad \times\left(\cos \theta_{k}-\cos \theta_{k-1}\right)\left(\sin \theta_{k-1}+\sin \theta_{k}\right)\left(\sin \varphi_{j}-\sin \varphi_{j-1}\right) \\
=-\frac{a^{5}}{6} \lim _{n \rightarrow \infty} \sum_{k=1}^{n} \sum_{j=1}^{k} \sum_{h=1}^{k}\left[\sin ^{2}\left(\frac{h}{n} \pi\right)+\sin ^{2}\left(\frac{k}{n} \pi\right) \sin \left(2 \frac{j}{n} \pi\right)+\cos ^{2}\left(\frac{k}{n} \pi\right)\right] \\
\quad \times\left[\sin \left(\frac{h}{n} \pi\right)-\sin \left(\frac{h-1}{n} \pi\right)\right]\left[\cos \left(\frac{k}{n} \pi\right)-\cos \left(\frac{k-1}{n} \pi\right)\right] \\
\quad \times\left[\sin \left(\frac{k-1}{n} \pi\right)+\sin \left(\frac{k}{n} \pi\right)\right]\left[\sin \left(2 \frac{j}{n} \pi\right)-\sin \left(2 \frac{j-1}{n} \pi\right)\right] \\
=0 .
\end{aligned}
$$

Since $\Delta_{j} z_{k-1, j}=0$ and $\Delta_{j} z_{k, j}=0$, the value of $\frac{1}{3} \lim _{n \rightarrow \infty} \sum_{k=1}^{n} \sum_{j=1}^{k} \sum_{i=1}^{j} q\left(x_{k, i}, y_{k, j}, z_{k, j}\right) \times$ $\Delta_{i} x_{k, i}\left(\Delta^{2} \sigma_{x}\right)_{k, j}$ is

$$
\begin{aligned}
& \frac{1}{3} \lim _{n \rightarrow \infty} \sum_{k=1}^{n} \sum_{j=1}^{k} \sum_{i=1}^{j} q\left(x_{k, i}, y_{k, j}, z_{k, j}\right) \Delta_{i} x_{k, i}\left(\Delta^{2} \sigma_{x}\right)_{k, j} \\
&=\frac{1}{6} \lim _{n \rightarrow \infty} \sum_{k=1}^{n} \sum_{j=1}^{k} \sum_{i=1}^{j}\left(x_{k, i}^{2}+y_{k, j}^{2}+z_{k, j}^{2}\right) \Delta_{i} x_{k, i}\left(\Delta_{k} y_{k, j} \Delta_{j} z_{k-1, j}+\Delta_{j} z_{k, j} \Delta_{k} y_{k, j-1}\right) \\
& \quad-\frac{1}{6} \lim _{n \rightarrow \infty} \sum_{k=1}^{n} \sum_{j=1}^{k} \sum_{i=1}^{j}\left(x_{k, i}^{2}+y_{k, j}^{2}+z_{k, j}^{2}\right) \Delta_{i} x_{k, i}\left(\Delta_{k} z_{k, j} \Delta_{j} y_{k-1, j}+\Delta_{j} y_{k, j} \Delta_{k} z_{k, j-1}\right) \\
&=0-\frac{a^{5}}{6} \lim _{n \rightarrow \infty} \sum_{k=1}^{n} \sum_{j=1}^{k} \sum_{i=1}^{j}\left(\sin ^{2} \theta_{k} \cos ^{2} \varphi_{i}+\sin ^{2} \theta_{k} \sin ^{2} \varphi_{j}+\cos ^{2} \theta_{k}\right) \\
& \times \sin \theta_{k}\left(\cos \varphi_{i}-\cos \varphi_{i-1}\right)\left(\cos \theta_{k}-\cos \theta_{k-1}\right) \\
& \times\left(\sin \theta_{k-1}+\sin \theta_{k}\right)\left(\sin \varphi_{j}-\sin \varphi_{j-1}\right) \\
&=-\frac{a^{5}}{6} \lim _{n \rightarrow \infty} \sum_{k=1}^{n} \sum_{j=1}^{k} \sum_{i=1}^{j}\left[\sin ^{2}\left(\frac{k}{n} \pi\right) \cos ^{2}\left(2 \frac{i}{n} \pi\right)\right. \\
&\left.+\sin ^{2}\left(\frac{k}{n} \pi\right) \sin ^{2}\left(2 \frac{j}{n} \pi\right)+\cos ^{2}\left(\frac{k}{n} \pi\right)\right]
\end{aligned}
$$




$$
\begin{aligned}
& \times \sin \left(\frac{k}{n} \pi\right)\left[\cos \left(2 \frac{i}{n} \pi\right)-\cos \left(2 \frac{i-1}{n} \pi\right)\right] \\
& \times\left[\cos \left(\frac{k}{n} \pi\right)-\cos \left(\frac{k-1}{n} \pi\right)\right]\left[\sin \left(\frac{k-1}{n} \pi\right)+\sin \left(\frac{k}{n} \pi\right)\right] \\
& \times\left[\sin \left(2 \frac{j}{n} \pi\right)-\sin \left(2 \frac{j-1}{n} \pi\right)\right] \\
& =\frac{2}{15} \pi a^{5} .
\end{aligned}
$$

Substituting (B.10), (B.11), (B.12), and (B.13) into (3.66), we obtain

$$
\frac{1}{3} \iiint_{D} q d x^{\prime \prime} d^{2} \sigma_{x}=\frac{4}{15} \pi a^{5}
$$

5. Calculation of (3.67) for Example 2 in detail.

Since $\Delta_{h} y_{0, h}=0$, the value of $\frac{1}{3} \lim _{n \rightarrow \infty} \sum_{k=1}^{n} \sum_{j=1}^{k} \sum_{h=1}^{k} q\left(x_{j, k}, y_{0, h}, z_{j, k}\right) \Delta_{h} y_{0, h}\left(\Delta^{2} \sigma_{y}\right)_{j, k}$ is

$$
\frac{1}{3} \lim _{n \rightarrow \infty} \sum_{k=1}^{n} \sum_{j=1}^{k} \sum_{h=1}^{k} q\left(x_{j, k}, y_{0, h}, z_{j, k}\right) \Delta_{h} y_{0, h}\left(\Delta^{2} \sigma_{y}\right)_{j, k}=0 .
$$

Since $\Delta_{k} z_{j-1, k}=0$ and $\Delta_{k} z_{j, k}=0$, the value of $\frac{1}{3} \lim _{n \rightarrow \infty} \sum_{k=1}^{n} \sum_{j=1}^{k} \sum_{i=1}^{j} q\left(x_{j, k}, y_{i, k}, z_{j, k}\right) \times$ $\Delta_{i} y_{i, k}\left(\Delta^{2} \sigma_{y}\right)_{j, k}$ is

$$
\begin{aligned}
& \frac{1}{3} \lim _{n \rightarrow \infty} \sum_{k=1}^{n} \sum_{j=1}^{k} \sum_{i=1}^{j} q\left(x_{j, k}, y_{i, k}, z_{j, k}\right) \Delta_{i} y_{i, k}\left(\Delta^{2} \sigma_{y}\right)_{j, k} \\
& =\frac{1}{6} \lim _{n \rightarrow \infty} \sum_{k=1}^{n} \sum_{j=1}^{k} \sum_{i=1}^{j}\left(x_{j, k}^{2}+y_{i, k}^{2}+z_{j, k}^{2}\right) \Delta_{i} y_{i, k}\left(\Delta_{j} z_{j, k} \Delta_{k} x_{j-1, k}+\Delta_{k} x_{j, k} \Delta_{j} z_{j, k-1}\right) \\
& -\frac{1}{6} \lim _{n \rightarrow \infty} \sum_{k=1}^{n} \sum_{j=1}^{k} \sum_{i=1}^{j}\left(x_{j, k}^{2}+y_{i, k}^{2}+z_{j, k}^{2}\right) \Delta_{i} y_{i, k}\left(\Delta_{j} x_{j, k} \Delta_{k} z_{j-1, k}+\Delta_{k} z_{j, k} \Delta_{j} x_{j, k-1}\right) \\
& =\frac{a^{5}}{6} \lim _{n \rightarrow \infty} \sum_{k=1}^{n} \sum_{j=1}^{k} \sum_{i=1}^{j}\left(\sin ^{2} \theta_{j} \cos ^{2} \varphi_{k}+\sin ^{2} \theta_{i} \sin ^{2} \varphi_{k}+\cos ^{2} \theta_{j}\right) \\
& \times\left(\sin \theta_{i}-\sin \theta_{i-1}\right) \sin \varphi_{k} \\
& \times\left(\cos \theta_{j}-\cos \theta_{j-1}\right)\left(\sin \theta_{j-1}+\sin \theta_{j}\right)\left(\cos \varphi_{k}-\cos \varphi_{k-1}\right)+0 \\
& =\frac{a^{5}}{6} \lim _{n \rightarrow \infty} \sum_{k=1}^{n} \sum_{j=1}^{k} \sum_{i=1}^{j}\left[\sin ^{2}\left(\frac{j}{n} \pi\right) \cos ^{2}\left(2 \frac{k}{n} \pi\right)\right. \\
& \left.+\sin ^{2}\left(\frac{i}{n} \pi\right) \sin ^{2}\left(2 \frac{k}{n} \pi\right)+\cos ^{2}\left(\frac{j}{n} \pi\right)\right] \\
& \times\left[\sin \left(\frac{i}{n} \pi\right)-\sin \left(\frac{i-1}{n} \pi\right)\right] \sin \left(2 \frac{k}{n} \pi\right) \\
& \times\left[\cos \left(\frac{j}{n} \pi\right)-\cos \left(\frac{j-1}{n} \pi\right)\right]\left[\sin \left(\frac{j-1}{n} \pi\right)+\sin \left(\frac{j}{n} \pi\right)\right]
\end{aligned}
$$




$$
\begin{aligned}
& \times\left[\cos \left(2 \frac{k}{n} \pi\right)-\cos \left(2 \frac{k-1}{n} \pi\right)\right] \\
= & \frac{2}{15} \pi a^{5} .
\end{aligned}
$$

Since $\Delta_{h} y_{h, 0}=0$, the value of $\frac{1}{3} \lim _{n \rightarrow \infty} \sum_{k=1}^{n} \sum_{j=1}^{k} \sum_{h=1}^{k} q\left(x_{k, j}, y_{h, 0}, z_{k, j}\right) \Delta_{h} y_{h, 0}\left(\Delta^{2} \sigma_{y}\right)_{k, j}$ is

$$
\frac{1}{3} \lim _{n \rightarrow \infty} \sum_{k=1}^{n} \sum_{j=1}^{k} \sum_{h=1}^{k} q\left(x_{k, j}, y_{h, 0}, z_{k, j}\right) \Delta_{h} y_{h, 0}\left(\Delta^{2} \sigma_{y}\right)_{k, j}=0 .
$$

Since $\Delta_{j} z_{k-1, j}=0$ and $\Delta_{j} z_{k, j}=0$, the value of $\frac{1}{3} \lim _{n \rightarrow \infty} \sum_{k=1}^{n} \sum_{j=1}^{k} \sum_{i=1}^{j} q\left(x_{k, j}, y_{k, i}, z_{k, j}\right) \times$ $\Delta_{i} y_{k, i}\left(\Delta^{2} \sigma_{y}\right)_{k, j}$ is

$$
\begin{aligned}
& \frac{1}{3} \lim _{n \rightarrow \infty} \sum_{k=1}^{n} \sum_{j=1}^{k} \sum_{i=1}^{j} q\left(x_{k, j}, y_{k, i}, z_{k, j}\right) \Delta_{i} y_{k, i}\left(\Delta^{2} \sigma_{y}\right)_{k, j} \\
& =\frac{1}{6} \lim _{n \rightarrow \infty} \sum_{k=1}^{n} \sum_{j=1}^{k} \sum_{i=1}^{j}\left(x_{k, j}^{2}+y_{k, i}^{2}+z_{k, j}^{2}\right) \Delta_{i} y_{k, i}\left(\Delta_{k} z_{k, j} \Delta_{j} x_{k-1, j}+\Delta_{j} x_{k, j} \Delta_{k} z_{k, j-1}\right) \\
& -\frac{1}{6} \lim _{n \rightarrow \infty} \sum_{k=1}^{n} \sum_{j=1}^{k} \sum_{i=1}^{j}\left(x_{k, j}^{2}+y_{k, i}^{2}+z_{k, j}^{2}\right) \Delta_{i} y_{k, i}\left(\Delta_{k} x_{k, j} \Delta_{j} z_{k-1, j}+\Delta_{j} z_{k, j} \Delta_{k} x_{k, j-1}\right) \\
& =\frac{a^{5}}{6} \lim _{n \rightarrow \infty} \sum_{k=1}^{n} \sum_{j=1}^{k} \sum_{i=1}^{j}\left(\sin ^{2} \theta_{k} \cos ^{2} \varphi_{j}+\sin ^{2} \theta_{k} \sin ^{2} \varphi_{i}+\cos ^{2} \theta_{k}\right) \\
& \times \sin \theta_{k}\left(\sin \varphi_{i}-\sin \varphi_{i-1}\right) \\
& \times\left(\cos \theta_{k}-\cos \theta_{k-1}\right)\left(\sin \theta_{k-1}+\sin \theta_{k}\right)\left(\cos \varphi_{j}-\cos \varphi_{j-1}\right)+0 \\
& =\frac{a^{5}}{6} \lim _{n \rightarrow \infty} \sum_{k=1}^{n} \sum_{j=1}^{k} \sum_{i=1}^{j}\left[\sin ^{2}\left(\frac{k}{n} \pi\right) \cos ^{2}\left(2 \frac{j}{n} \pi\right)\right. \\
& \left.+\sin ^{2}\left(\frac{k}{n} \pi\right) \sin ^{2}\left(2 \frac{i}{n} \pi\right)+\cos ^{2}\left(\frac{k}{n} \pi\right)\right] \\
& \times \sin \left(\frac{k}{n} \pi\right)\left[\sin \left(2 \frac{i}{n} \pi\right)-\sin \left(2 \frac{i-1}{n} \pi\right)\right] \\
& \times\left[\cos \left(\frac{k}{n} \pi\right)-\cos \left(\frac{k-1}{n} \pi\right)\right]\left[\sin \left(\frac{k-1}{n} \pi\right)+\sin \left(\frac{k}{n} \pi\right)\right] \\
& \times\left[\cos \left(2 \frac{j}{n} \pi\right)-\cos \left(2 \frac{j-1}{n} \pi\right)\right] \\
& =\frac{2}{15} \pi a^{5} \text {. }
\end{aligned}
$$

Substituting (B.15), (B.16), (B.17), and (B.18) into (3.67), we obtain

$$
\frac{1}{3} \iiint_{D} q d y^{\prime \prime} d^{2} \sigma_{y}=\frac{4}{15} \pi a^{5}
$$

6. Calculation of (3.68) for Example 2 in detail. 
Since $\Delta_{h} z_{0, h}=0$, the value of $\frac{1}{3} \lim _{n \rightarrow \infty} \sum_{k=1}^{n} \sum_{j=1}^{k} \sum_{h=1}^{k} q\left(x_{j, k}, y_{j, k}, z_{0, h}\right) \Delta_{h} z_{0, h}\left(\Delta^{2} \sigma_{z}\right)_{j, k}$ is

$$
\frac{1}{3} \lim _{n \rightarrow \infty} \sum_{k=1}^{n} \sum_{j=1}^{k} \sum_{h=1}^{k} q\left(x_{j, k}, y_{j, k}, z_{0, h}\right) \Delta_{h} z_{0, h}\left(\Delta^{2} \sigma_{z}\right)_{j, k}=0 .
$$

The value of $\frac{1}{3} \lim _{n \rightarrow \infty} \sum_{k=1}^{n} \sum_{j=1}^{k} \sum_{i=1}^{j} q\left(x_{j, k}, y_{j, k}, z_{i, k}\right) \Delta_{i} z_{i, k}\left(\Delta^{2} \sigma_{z}\right)_{j, k}$ is

$$
\begin{aligned}
& \frac{1}{3} \lim _{n \rightarrow \infty} \sum_{k=1}^{n} \sum_{j=1}^{k} \sum_{i=1}^{j} q\left(x_{j, k}, y_{j, k}, z_{i, k}\right) \Delta_{i} z_{i, k}\left(\Delta^{2} \sigma_{z}\right)_{j, k} \\
& =\frac{1}{6} \lim _{n \rightarrow \infty} \sum_{k=1}^{n} \sum_{j=1}^{k} \sum_{i=1}^{j}\left(x_{j, k}^{2}+y_{j, k}^{2}+z_{i, k}^{2}\right) \Delta_{i} z_{i, k}\left(\Delta_{j} x_{j, k} \Delta_{k} y_{j-1, k}+\Delta_{k} y_{j, k} \Delta_{j} x_{j, k-1}\right) \\
& -\frac{1}{6} \lim _{n \rightarrow \infty} \sum_{k=1}^{n} \sum_{j=1}^{k} \sum_{i=1}^{j}\left(x_{j, k}^{2}+y_{j, k}^{2}+z_{i, k}^{2}\right) \Delta_{i} z_{i, k}\left(\Delta_{j} y_{j, k} \Delta_{k} x_{j-1, k}+\Delta_{k} x_{j, k} \Delta_{j} y_{j, k-1}\right) \\
& =\frac{a^{5}}{6} \lim _{n \rightarrow \infty} \sum_{k=1}^{n} \sum_{j=1}^{k} \sum_{i=1}^{j}\left(\sin ^{2} \theta_{j} \cos ^{2} \varphi_{k}+\sin ^{2} \theta_{j} \sin ^{2} \varphi_{k}+\cos ^{2} \theta_{i}\right)\left(\cos \theta_{i}-\cos \theta_{i-1}\right) \\
& \times\left(\sin \theta_{j}-\sin \theta_{j-1}\right)\left(\sin \varphi_{k}-\sin \varphi_{k-1}\right)\left(\cos \varphi_{k} \sin \theta_{j-1}+\cos \varphi_{k-1} \sin \theta_{j}\right) \\
& -\frac{a^{5}}{6} \lim _{n \rightarrow \infty} \sum_{k=1}^{n} \sum_{j=1}^{k} \sum_{i=1}^{j}\left(\sin ^{2} \theta_{j} \cos ^{2} \varphi_{k}+\sin ^{2} \theta_{j} \sin ^{2} \varphi_{k}+\cos ^{2} \theta_{i}\right) \\
& \times\left(\cos \theta_{i}-\cos \theta_{i-1}\right) \\
& \times\left(\sin \theta_{j}-\sin \theta_{j-1}\right)\left(\cos \varphi_{k}-\cos \varphi_{k-1}\right)\left(\sin \varphi_{k} \sin \theta_{j-1}+\sin \varphi_{k-1} \sin \theta_{j}\right) \\
& =\frac{a^{5}}{6} \lim _{n \rightarrow \infty} \sum_{k=1}^{n} \sum_{j=1}^{k} \sum_{i=1}^{j}\left[\sin ^{2}\left(\frac{j}{n} \pi\right) \cos ^{2}\left(2 \frac{k}{n} \pi\right)\right. \\
& \left.+\sin ^{2}\left(\frac{j}{n} \pi\right) \sin ^{2}\left(2 \frac{k}{n} \pi\right)+\cos ^{2}\left(\frac{i}{n} \pi\right)\right] \\
& \times\left[\cos \left(\frac{i}{n} \pi\right)-\cos \left(\frac{i-1}{n} \pi\right)\right]\left[\sin \left(\frac{j}{n} \pi\right)-\sin \left(\frac{j-1}{n} \pi\right)\right] \\
& \times\left[\sin \left(2 \frac{k}{n} \pi\right)-\sin \left(2 \frac{k-1}{n} \pi\right)\right] \\
& \times\left[\cos \left(2 \frac{k}{n} \pi\right) \sin \left(\frac{j-1}{n} \pi\right)+\cos \left(2 \frac{k-1}{n} \pi\right) \sin \left(\frac{j}{n} \pi\right)\right] \\
& -\frac{a^{5}}{6} \lim _{n \rightarrow \infty} \sum_{k=1}^{n} \sum_{j=1}^{k} \sum_{i=1}^{j}\left[\sin ^{2}\left(\frac{j}{n} \pi\right) \cos ^{2}\left(2 \frac{k}{n} \pi\right)\right. \\
& \left.+\sin ^{2}\left(\frac{j}{n} \pi\right) \sin ^{2}\left(2 \frac{k}{n} \pi\right)+\cos ^{2}\left(\frac{i}{n} \pi\right)\right] \\
& \times\left[\cos \left(\frac{i}{n} \pi\right)-\cos \left(\frac{i-1}{n} \pi\right)\right]\left[\sin \left(\frac{j}{n} \pi\right)-\sin \left(\frac{j-1}{n} \pi\right)\right] \\
& \times\left[\cos \left(2 \frac{k}{n} \pi\right)-\cos \left(2 \frac{k-1}{n} \pi\right)\right]
\end{aligned}
$$




$$
\begin{aligned}
& \times\left[\sin \left(2 \frac{k}{n} \pi\right) \sin \left(\frac{j-1}{n} \pi\right)+\sin \left(2 \frac{k-1}{n} \pi\right) \sin \left(\frac{j}{n} \pi\right)\right] \\
= & \frac{7}{2,880} \pi a^{5}+\frac{37}{2,880} \pi a^{5} \\
= & \frac{11}{720} \pi a^{5} .
\end{aligned}
$$

The value of $\frac{1}{3} \lim _{n \rightarrow \infty} \sum_{k=1}^{n} \sum_{j=1}^{k} \sum_{h=1}^{k} q\left(x_{k, j}, y_{k, j}, z_{h, 0}\right) \Delta_{h} z_{h, 0}\left(\Delta^{2} \sigma_{z}\right)_{k, j}$ is

$$
\begin{aligned}
& \frac{1}{3} \lim _{h \rightarrow \infty} \sum_{k=1}^{n} \sum_{j=1}^{k} \sum_{h=1}^{k} q\left(x_{k, j}, y_{k, j}, z_{h, 0}\right) \Delta_{h} z_{h, 0}\left(\Delta^{2} \sigma_{z}\right)_{k, j} \\
& =\frac{1}{6} \lim _{n \rightarrow \infty} \sum_{k=1}^{n} \sum_{j=1}^{k} \sum_{h=1}^{k}\left(x_{k, j}^{2}+y_{k, j}^{2}+z_{h, 0}^{2}\right) \Delta_{h} z_{h, 0}\left(\Delta_{k} x_{k, j} \Delta_{j} y_{k-1, j}+\Delta_{j} y_{k, j} \Delta_{k} x_{k, j-1}\right) \\
& -\frac{1}{6} \lim _{n \rightarrow \infty} \sum_{k=1}^{n} \sum_{j=1}^{k} \sum_{h=1}^{k}\left(x_{k, j}^{2}+y_{k, j}^{2}+z_{h, 0}^{2}\right) \Delta_{h} z_{h, 0}\left(\Delta_{k} y_{k, j} \Delta_{j} x_{k-1, j}+\Delta_{j} x_{k, j} \Delta_{k} y_{k, j-1}\right) \\
& =\frac{a^{5}}{6} \lim _{n \rightarrow \infty} \sum_{k=1}^{n} \sum_{j=1}^{k} \sum_{h=1}^{k}\left(\sin ^{2} \theta_{k} \cos ^{2} \varphi_{j}+\sin ^{2} \theta_{k} \sin ^{2} \varphi_{j}+\cos ^{2} \theta_{h}\right)\left(\cos \theta_{h}-\cos \theta_{h-1}\right) \\
& \times\left(\sin \theta_{k}-\sin \theta_{k-1}\right)\left(\sin \varphi_{j}-\sin \varphi_{j-1}\right)\left(\cos \varphi_{j} \sin \theta_{k-1}+\cos \varphi_{j-1} \sin \theta_{k}\right) \\
& -\frac{a^{5}}{6} \lim _{n \rightarrow \infty} \sum_{k=1}^{n} \sum_{j=1}^{k} \sum_{h=1}^{k}\left(\sin ^{2} \theta_{k} \cos ^{2} \varphi_{j}+\sin ^{2} \theta_{k} \sin ^{2} \varphi_{j}+\cos ^{2} \theta_{h}\right)\left(\cos \theta_{h}-\cos \theta_{h-1}\right) \\
& \times\left(\sin \theta_{k}-\sin \theta_{k-1}\right)\left(\cos \varphi_{j}-\cos \varphi_{j-1}\right)\left(\sin \varphi_{j} \sin \theta_{k-1}+\sin \varphi_{j-1} \sin \theta_{k}\right) \\
& =\frac{a^{5}}{6} \lim _{n \rightarrow \infty} \sum_{k=1}^{n} \sum_{j=1}^{k} \sum_{h=1}^{k}\left[\sin ^{2}\left(\frac{k}{n} \pi\right) \cos ^{2}\left(2 \frac{j}{n} \pi\right)\right. \\
& \left.+\sin ^{2}\left(\frac{k}{n} \pi\right) \sin ^{2}\left(2 \frac{j}{n} \pi\right)+\cos ^{2}\left(\frac{h}{n} \pi\right)\right] \\
& \times\left[\cos \left(\frac{h}{n} \pi\right)-\cos \left(\frac{h-1}{n} \pi\right)\right]\left[\sin \left(\frac{k}{n} \pi\right)-\sin \left(\frac{k-1}{n} \pi\right)\right] \\
& \times\left[\sin \left(2 \frac{j}{n} \pi\right)-\sin \left(2 \frac{j-1}{n} \pi\right)\right] \\
& \times\left[\cos \left(2 \frac{j}{n} \pi\right) \sin \left(\frac{k-1}{n} \pi\right)+\cos \left(2 \frac{j-1}{n} \pi\right) \sin \left(\frac{k}{n} \pi\right)\right] \\
& -\frac{a^{5}}{6} \lim _{n \rightarrow \infty} \sum_{k=1}^{n} \sum_{j=1}^{k} \sum_{h=1}^{k}\left[\sin ^{2}\left(\frac{k}{n} \pi\right) \cos ^{2}\left(2 \frac{j}{n} \pi\right)\right. \\
& \left.+\sin ^{2}\left(\frac{k}{n} \pi\right) \sin ^{2}\left(2 \frac{j}{n} \pi\right)+\cos ^{2}\left(\frac{h}{n} \pi\right)\right] \\
& \times\left[\cos \left(\frac{h}{n} \pi\right)-\cos \left(\frac{h-1}{n} \pi\right)\right]\left[\sin \left(\frac{k}{n} \pi\right)-\sin \left(\frac{k-1}{n} \pi\right)\right] \\
& \times\left[\cos \left(2 \frac{j}{n} \pi\right)-\cos \left(2 \frac{j-1}{n} \pi\right)\right]
\end{aligned}
$$




$$
\begin{aligned}
& \times\left[\sin \left(2 \frac{j}{n} \pi\right) \sin \left(\frac{k-1}{n} \pi\right)+\sin \left(2 \frac{j-1}{n} \pi\right) \sin \left(\frac{k}{n} \pi\right)\right] \\
= & \frac{377}{2,880} \pi a^{5}+\frac{347}{2,880} \pi a^{5} \\
= & \frac{181}{720} \pi a^{5} .
\end{aligned}
$$

Since $\Delta_{i} z_{k, i}=0$, the value of $\frac{1}{3} \lim _{n \rightarrow \infty} \sum_{k=1}^{n} \sum_{j=1}^{k} \sum_{i=1}^{j} q\left(x_{k, j}, y_{k, j}, z_{k, i}\right) \Delta_{i} z_{k, i}\left(\Delta^{2} \sigma_{z}\right)_{k, j}$ is

$$
\frac{1}{3} \lim _{n \rightarrow \infty} \sum_{k=1}^{n} \sum_{j=1}^{k} \sum_{i=1}^{j} q\left(x_{k, j}, y_{k, j}, z_{k, i}\right) \Delta_{i} z_{k, i}\left(\Delta^{2} \sigma_{z}\right)_{k, j}=0 .
$$

Substituting (B.20), (B.21), (B.22), and (B.23) into (3.68), we obtain

$$
\frac{1}{3} \iiint_{D} q d z^{\prime \prime} d^{2} \sigma_{z}=\frac{4}{15} \pi a^{5}
$$

\section{Competing interests}

The author declares that he has no competing interests.

\section{Author's contributions}

The author is the only person who is responsible to this work.

\section{Acknowledgements}

The author sincerely thanks the reviewers for their valuable suggestions and useful comments.

Received: 15 July 2013 Accepted: 26 February 2014 Published: 17 Mar 2014

\section{References}

1. Lebesgue, H: Intégrale, longueur, aire. Thèses présentées à la Faculté des sciences de Paris pour obtenir le grade de Docteur ès sciences mathématiques. Bernardoni de C. Rebeschini, Milano (1902). Ann. Mat. Pura Appl. 7, 231-359 (1902)

2. Lebesgue, H: Leçon sur L'intégration et la Recherche des Fonctions Primitives. Gauthier-Villars, Paris (1904)

3. Bramble, JH, Zlámal, M: Triangular elements in the finite element method. Math. Comput. 24(112), 809-820 (1970)

4. Proenca, $\mathrm{H}$, Filipe, S: Combining rectangular and triangular image regions to perform real-time face detection. In: Signal Processing, 2008. ICSP 2008. 9th International Conference on, pp. $903-908$ (2008) doi:10.1109/ICOSP.2008.4697274

5. Tokunaga, K: The curl theorem of a triangular integral. Adv. Differ. Equ. 2012, 23 (2012). doi:10.1186/1687-1847-2012-23

6. Tokunaga, K: The divergence theorem of a triangular integral. Adv. Differ. Equ. 2012, 168 (2012). doi:10.1186/1687-1847-2012-168

7. Cartan, É: Sur l'intégration des systèmes d'équations aux différentielles totales. Ann. Sci. Éc. Norm. Super. 18, 241-311 (1901)

10.1186/1687-1847-2014-89

Cite this article as: Tokunaga: Triangular integrals for 2-, 3- and 4-variable functions. Advances in Difference Equations 2014, 2014:89 